\title{
Towards an Understanding of the Immigrant Experience: Social-cognitive Processes of Identity Development during Cultural Transition
}

\author{
by \\ Ágnes Szabó \\ A thesis submitted to Victoria University of Wellington \\ in fulfilment of the requirements for the degree of \\ Doctor of Philosophy in Psychology
}

Victoria University of Wellington 


\section{Acknowledgments}

First and foremost I would like to thank my primary supervisor Prof Colleen Ward. It has been an honour being your student. Thank you for your guidance, continuous encouragement, attention, and care throughout my $\mathrm{PhD}$ studies. I am very grateful that you allowed me to be creative and work in my own way. Most importantly, I would like to thank you for showing me every day how to become a successful researcher and a good academic by example.

I would like to express my special appreciation and thanks to my secondary supervisor Prof Garth Fletcher. Thank you for your support, invaluable comments and statistical guidance. I owe my supervisors a great deal of gratitude for opening many doors for me in the academic world. I cannot thank you enough for your help in advancing my career.

I also thank Nguyen Luu Lan Anh and Szabó Mónika for introducing me to the field of crosscultural psychology. I could not have completed my $\mathrm{PhD}$ if you had not provided me with training of such high quality. Thank you for your support and faith in me.

This acknowledgment would not be complete without mentioning both current and former members of the Centre for Applied Cross-cultural Research, a wonderful group of people who have always inspired and encouraged me.

- To Claudia Recker for taking me under her wing when I arrived to Wellington.

- To Adrienne Girling for being my friend and helping me to overcome both emotional and academic obstacles.

- To Laina Isler for her endless energy, enthusiasm, and passion. You are one of the kindest and funniest people I know. Thank you for bursting into dance with me to celebrate significant results, pretty graphs, and good model fits. You made my days at university so much more enjoyable.

- To Tamara Khair for being a kind, considerate and tolerant office mate. It is never easy to share an office with a finishing PhD student. I know that I have not always been the best companion, but I hope that I can show you that I am a much nicer, happier and funnier person than the one you got to know over the last one and a half years.

- To Reneeta Mogan for inventing Wednesday baking, sharing laughter, and giving me moral support when needed. 
- My gratitude is also extended to everyone involved in CACR for the shared knowledge and the valuable conversations, including (but not limited to) Alicia Bethel, Angela Robinson, Clara Cantal, Jovana Balanovic, Pollyanne Diniz, and Sara Morgan.

A big thank you to everyone who participated in my studies. Your time and effort made an essential contribution to my $\mathrm{PhD}$ research.

I would also like to give a heartfelt, special thanks to my immediate and extended family. Words cannot express how grateful I am to my parents who encouraged and supported me in all my pursuits. Thank you for teaching me the value of hard work and persistence, and for all the sacrifices you made to ensure I received the best education. I love you very much. To my brother, who has always expressed how proud he is of me, it means so much to me.

Last but not least, I would like to thank Roland for embarking upon this journey with me three years ago. I know that it has been extremely challenging for you, and I am unspeakably grateful for your unconditional love, support, and patience. You were my rock during this process. Thank you for giving me a home and bringing stability, balance and peace of mind into my life. 


\section{General abstract}

In a highly globalized world, where more than 200 million people are living as international migrants (OECD, 2013), continuously confronting and negotiating the demands of at least two different cultures, there is a growing need to understand how cultural transition affects the identity of immigrants and explore the factors that can potentially contribute to the development of a positive, coherent, and consolidated immigrant identity. The current research programme integrates aspects of developmental and cross-cultural theory to gain a deeper insight in to the dynamics of identity formation and the processes involved in identity reconstruction during cultural transition from the very early stages of acculturation. Drawing from previous work on identity styles by Berzonsky $(1989,2011)$, chapter two introduces a new version of the Identity Style Inventory assessing social-cognitive information processing strategies during cultural transition (ISI-CT). The ISI-CT incorporates five distinct identity styles (analytical informational, exploratory informational, normative to the country of origin, normative to the host society, and diffuse-avoidant) and a measure of identity commitment. Three studies are presented reporting the construction of the ISI-CT and the examination of its factorial structure (study 1 and study 2), the convergent and discriminant validity of the assessment tool (study 1), and longitudinal relationships among subscales (study 3). Chapter three extends Berzonsky's theorizing to acculturation research and tests a model of identity development with a diverse sample of newly arrived immigrants $(N=218)$. Results indicate that social-cognitive identity processing styles facilitate both positive and negative pathways to immigrant identity outcomes and highlight the central role of identity commitment during acculturation identity crises. In chapter four is presented a six-month longitudinal study that investigates the appraisal (threat or challenge) of identity stress in immigrants, the capacity of identity processing styles in reducing the negative effects of acculturative identity stress, and the prediction of psychological adjustment over time. Longitudinal interaction effects revealed that the analytical and exploratory informational styles enhanced the positive effects of challenge appraisal on psychological adjustment over time. In contrast, styles involving avoidance and normative orientation to one's country of origin dampened the positive effects of challenge appraisal on psychological adjustment six months later. In sum, the series of studies reported in the present thesis draw attention to the central role of social-cognitive identity styles in terms of identity development, acculturative stress management, and longterm psychological adaptation of recent immigrants. The findings of the research programme have important implications for acculturation theory and research by highlighting the benefits 
of the developmental approach in the understanding of the immigrant experience. In addition, potential avenues for applications and future research are discussed. 


\section{Table of Contents}

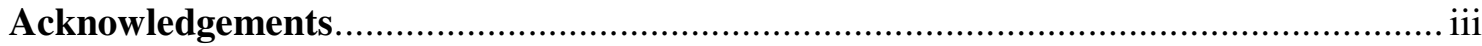

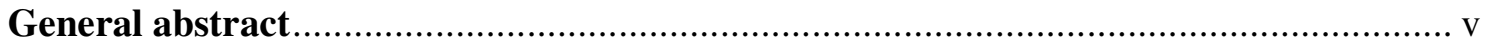

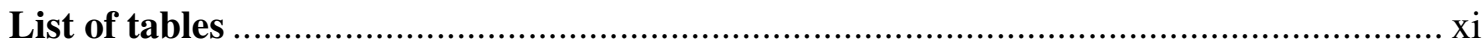

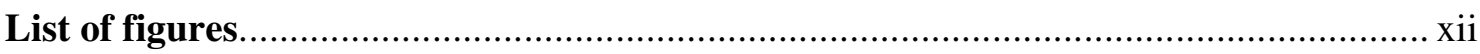

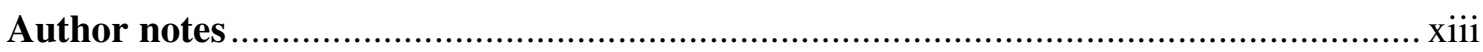

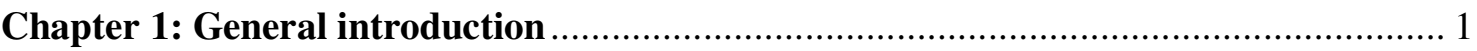

Migration and changing demographics in the contemporary world.................................. 3

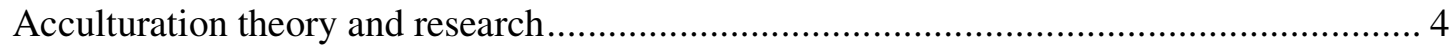

Acculturation and cross-cultural adaptation.......................................................... 4

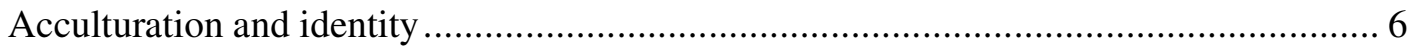

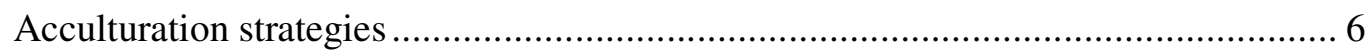

Ethnic and cultural identity integration .......................................................... 7

Evaluating theory and research on acculturation and identity ............................. 8

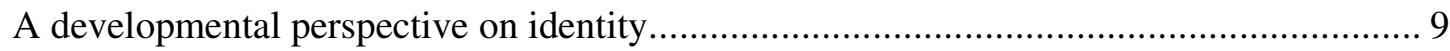

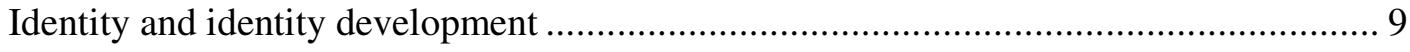

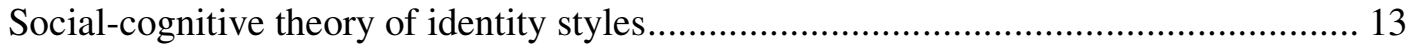

Identity development in acculturating individuals/minority groups............................ 14

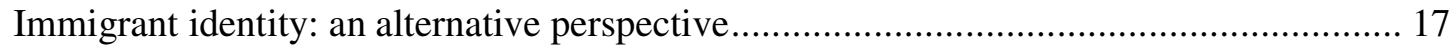

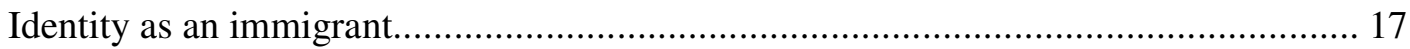

Applying developmental theory to immigrant identity............................................ 18

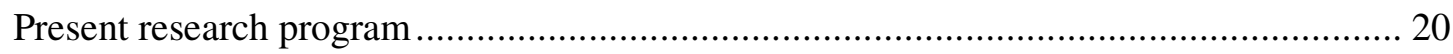

Chapter 2: Identity processing styles during cultural transition .............................. 25

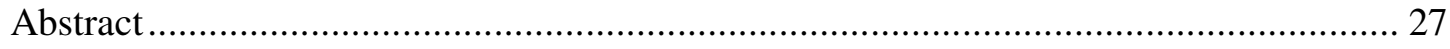

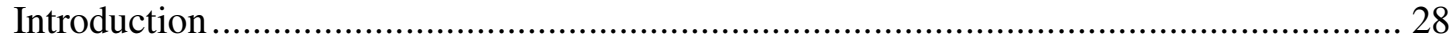

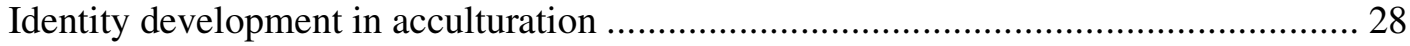

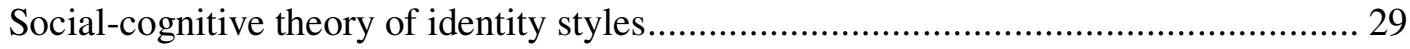

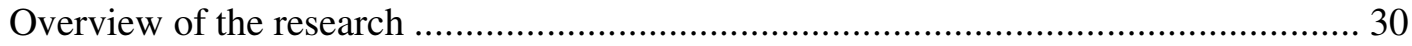

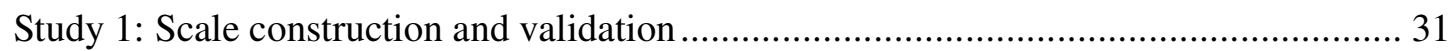

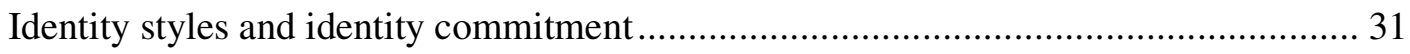

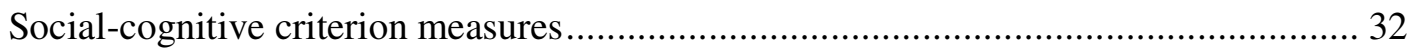

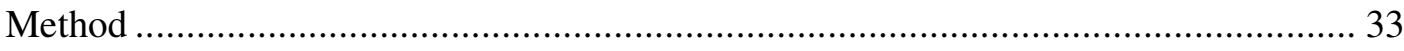

Construction of the Identity Style Inventory - Cultural Transition Version .............. 33

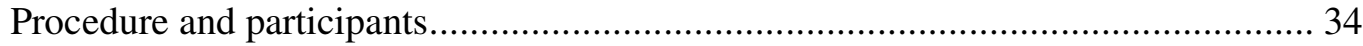




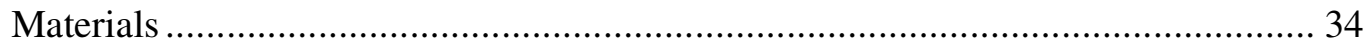

Identity Style Inventory - Cultural Transition Version ..................................... 34

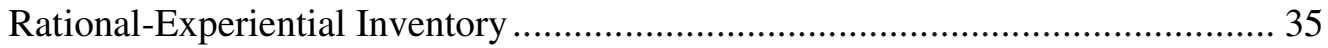

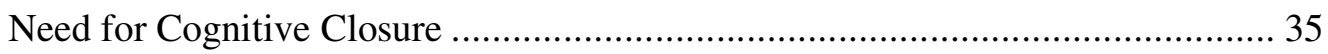

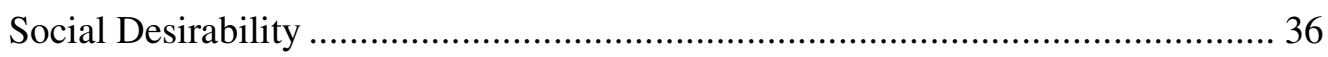

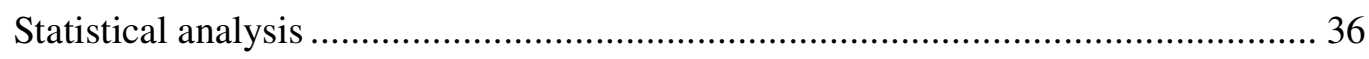

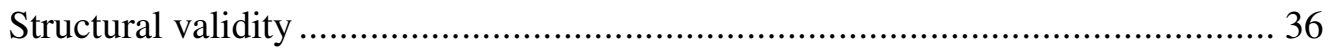

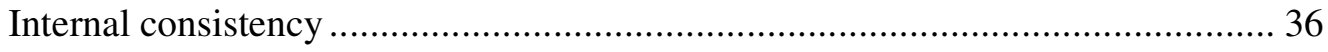

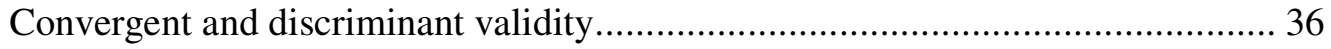

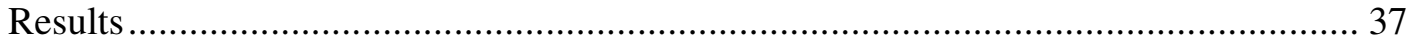

Parallel analysis and exploratory factor analysis: identity styles........................... 37

Principal component analysis: identity commitment .......................................... 39

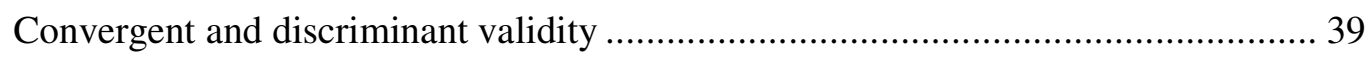

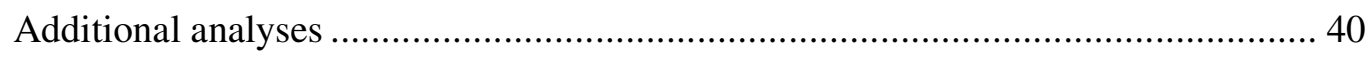

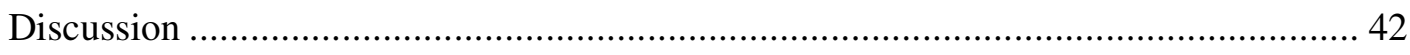

Structure of the Identity Style Inventory - Cultural Transition Version ................... 42

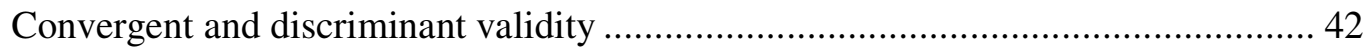

Study 2: Confirming the factor structure of the ISI-CT …............................................ 44

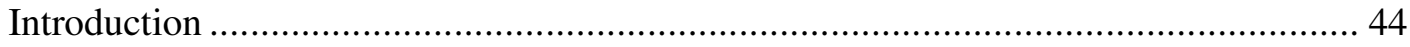

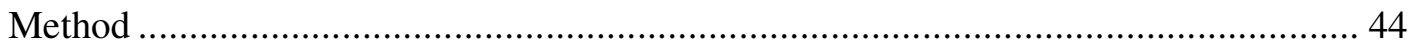

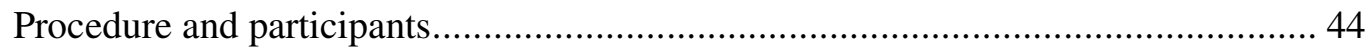

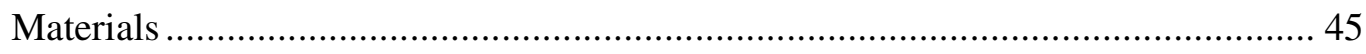

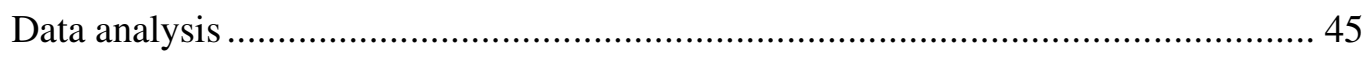

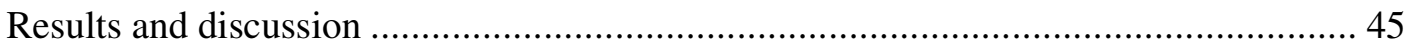

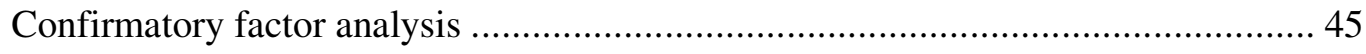

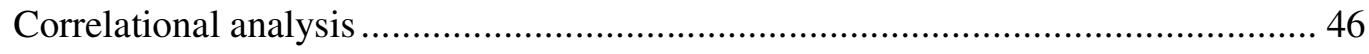

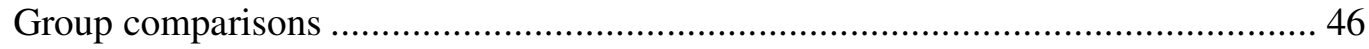

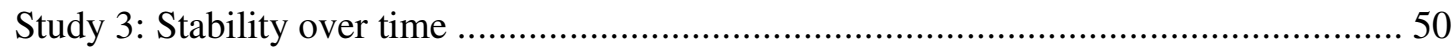

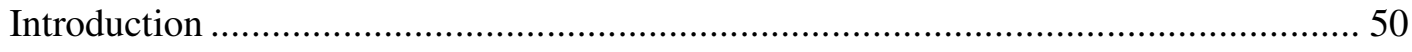

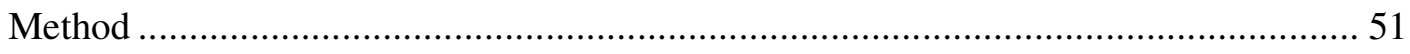

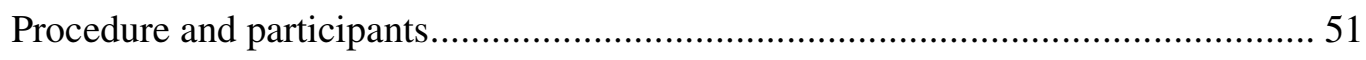

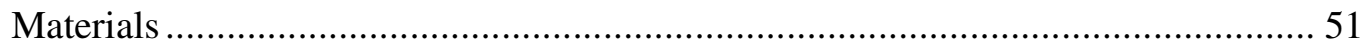

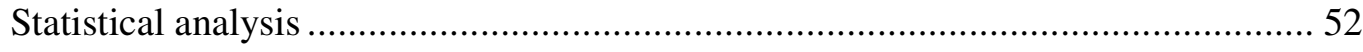

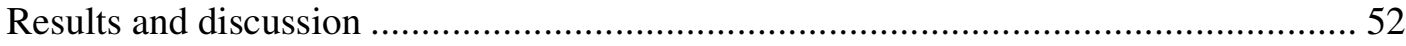

viii 


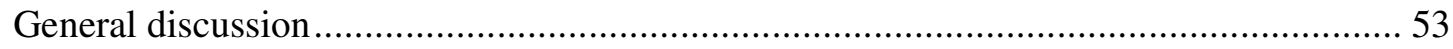

Limitations, future directions and concluding comments ........................................ 54

Chapter 3: Identity development during cultural transition................................. 57

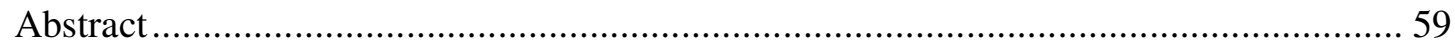

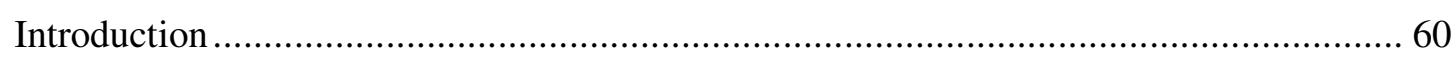

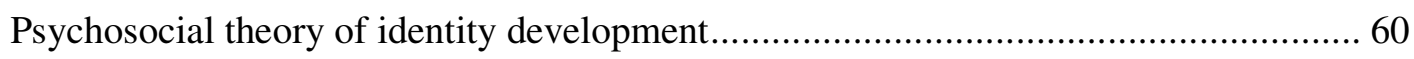

Identity styles: social-cognitive processes of identity development........................... 61

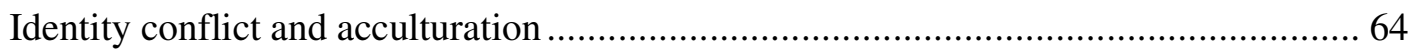

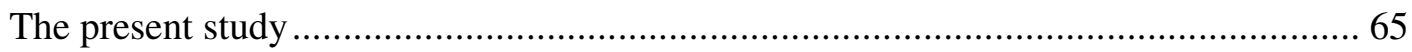

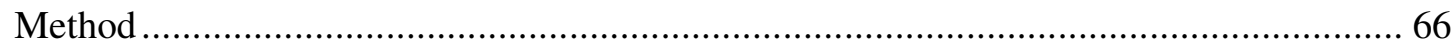

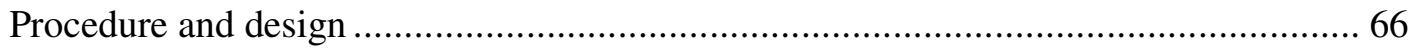

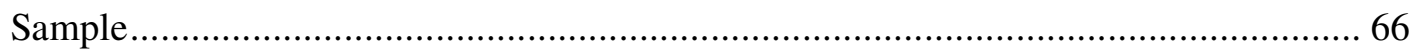

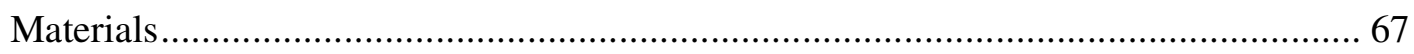

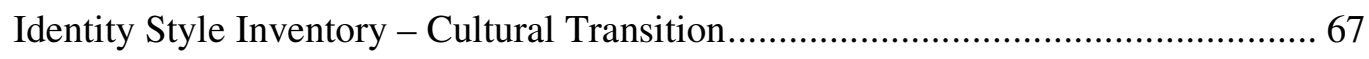

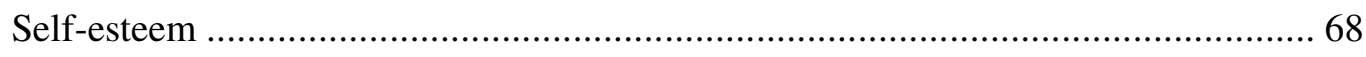

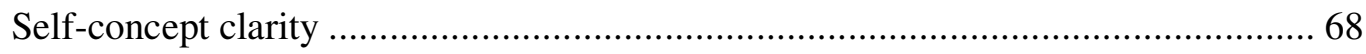

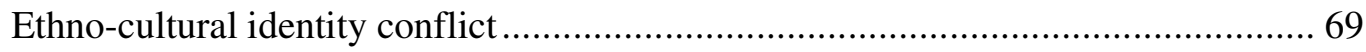

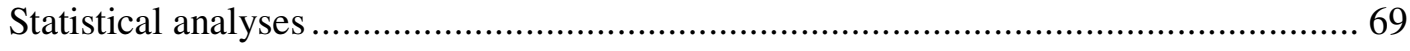

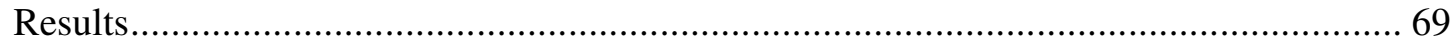

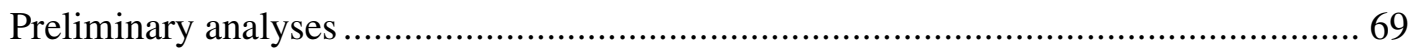

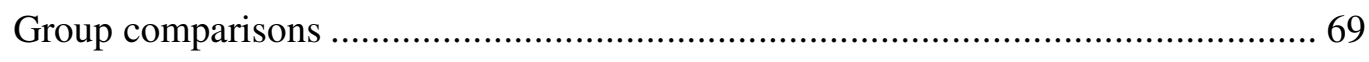

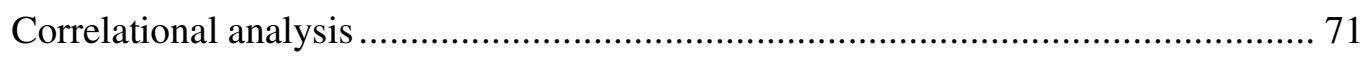

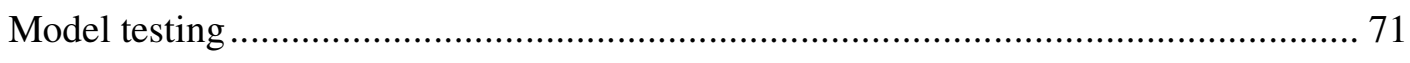

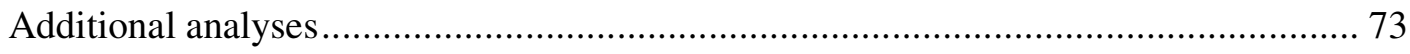

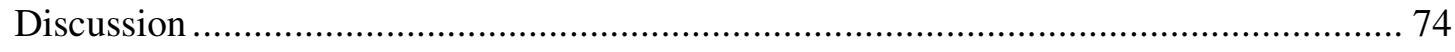

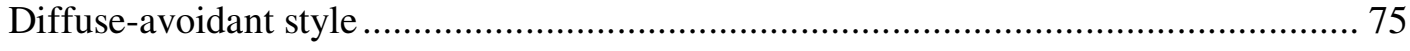

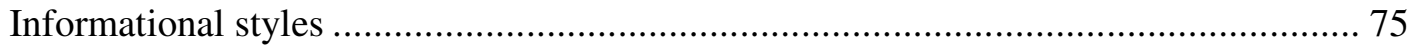

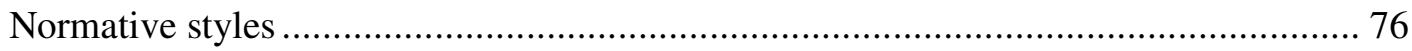

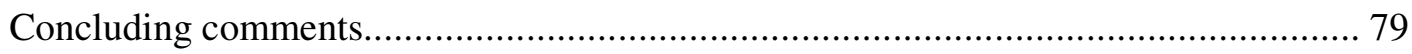

Chapter 4: Negotiating identity stress during cultural transition ........................... 81

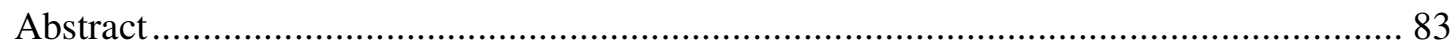

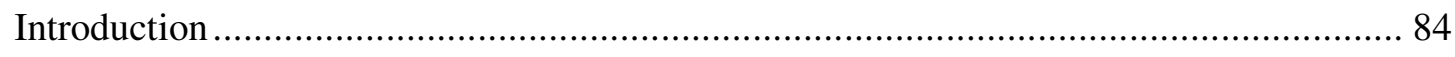

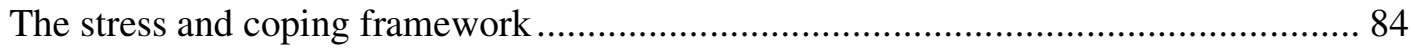

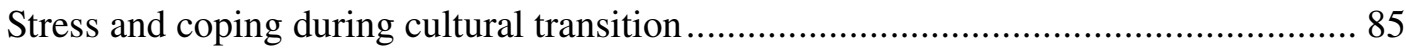




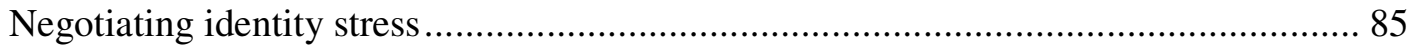

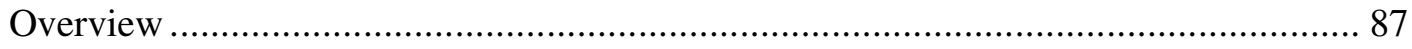

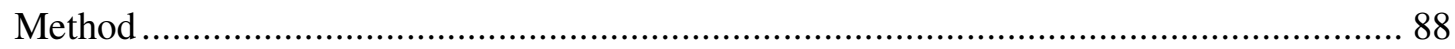

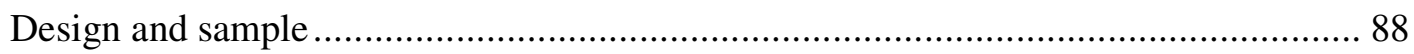

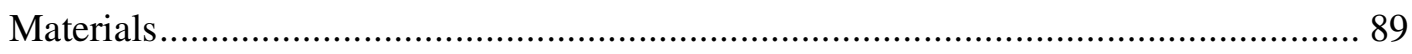

Identity Style Inventory - Cultural Transition Version....................................... 89

Primary Appraisal of Identity Threats ........................................................... 91

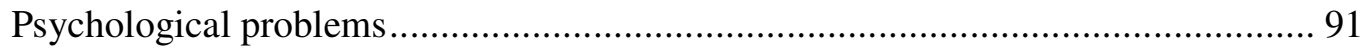

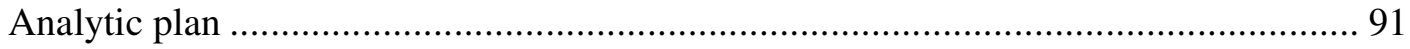

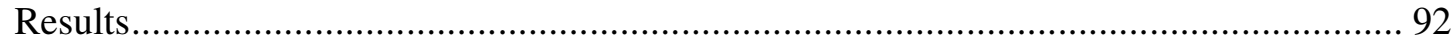

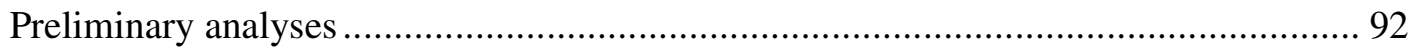

Relationships between demographic variables and the dependent measure ............. 92

Correlations among the variables of interest ................................................... 92

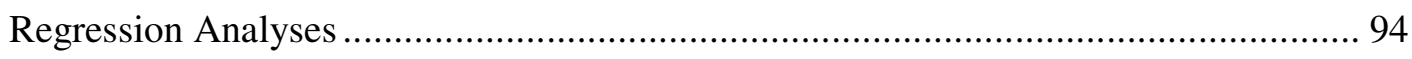

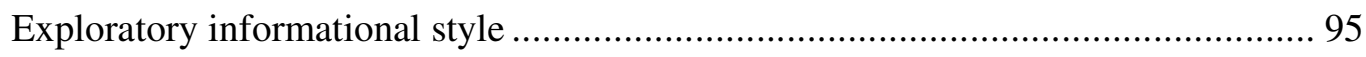

Analytical informational style ................................................................ 95

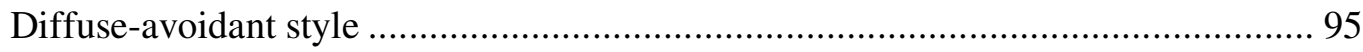

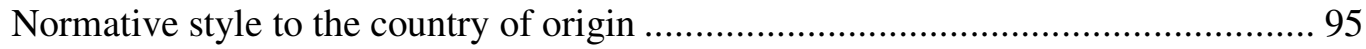

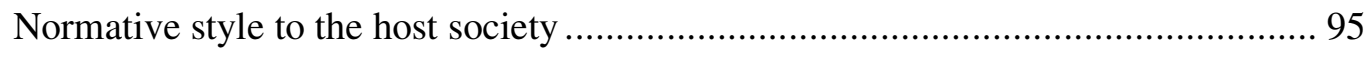

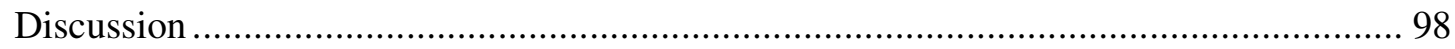

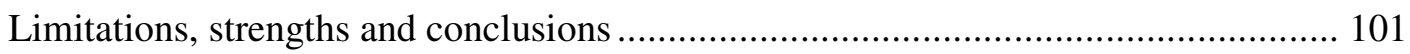

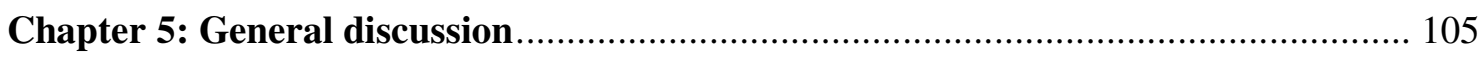

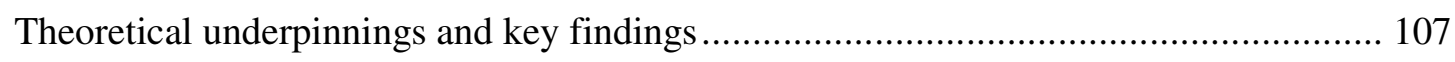

Examining acculturation and identity through the lens of developmental theory ......... 107

Examining acculturation and identity over time.................................................. 108

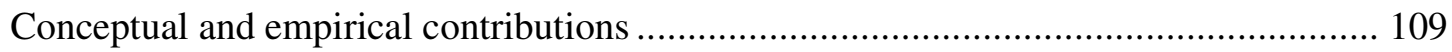

What can acculturation research offer to developmental theory? ............................... 109

The benefits of applying developmental theory in the context of acculturation research

Immigrant identity: an alternative framework.............................................. 112

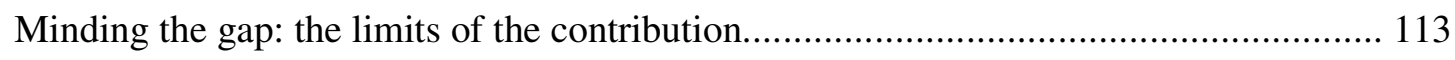

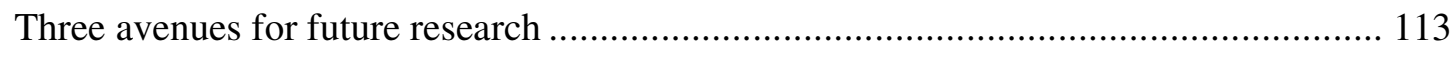

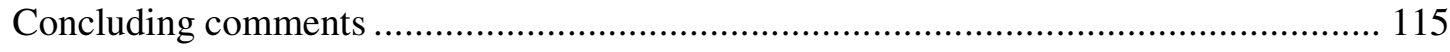

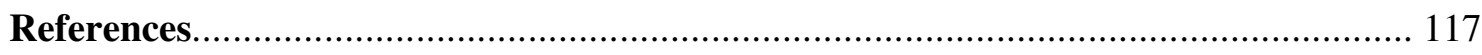




\section{List of Tables}

Table 2.1. Identity Style Inventory - Cultural Transition Version: Factor loadings and descriptive statistics (Study 1)

Table 2.2. Identity Commitment Scale: factor loadings, item-total correlations, and

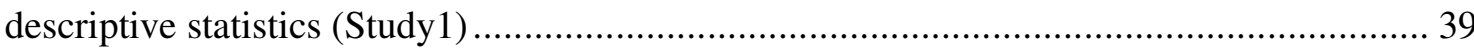

Table 2.3. Descriptive statistics and correlations among identity styles, criterion

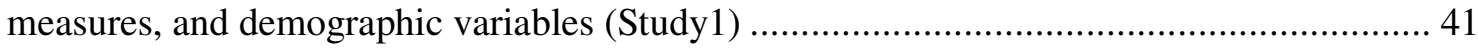

Table 2.4. $\quad$ Fit indices for each model and model comparisons (Study 2) ..................... 48

Table 2.5. Descriptive statistics, reliability and intercorrelations of the ISI-CT subscales (Study 2) 48

Table 3.1. Descriptive statistics and correlations among the variables of interest ......... 70 Table 3.2. Indirect effects between identity styles and identity outcomes through identity

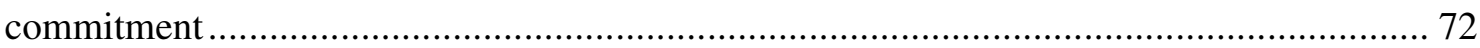

Table 3.3. Hierarchical regression of the prediction of self-esteem........................... 74

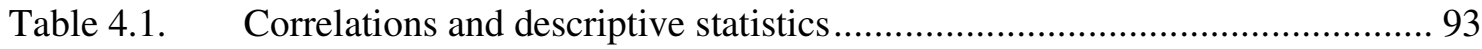

Table 4.2. Hierarchical regression of the prediction of change in psychological problems over time. 94 


\section{List of Figures}

Figure 1.1. The ABC model of acculturation............................................................ 5

Figure 1.2. Hierarchical model of identity ........................................................... 12

Figure 2.1. Confirmatory factor analysis of the identity style subscales of the ISI-CT .... 49

Figure 2.2. Confirmatory factor analysis of the identity commitment subscale of the ISI-

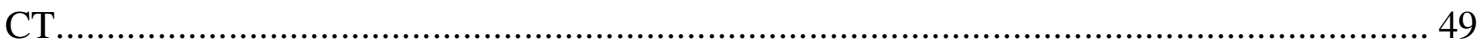

Figure 2.3. Cross-lagged model testing the temporal stability of the identity styles ........ 52

Figure 3.1. Proposed model of immigrant identity development in cultural transition..... 65

Figure 3.2. Path model testing the proposed theoretical model .................................... 72

Figure 3.3. Identity commitment buffers the negative impact of normative orientation to

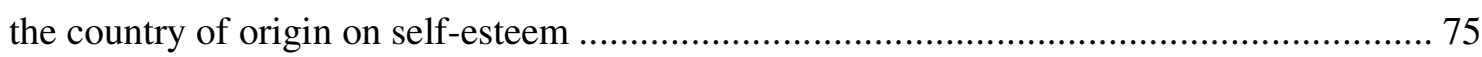

Figure 4.1. The exploratory informational style at time 1 enhanced the positive effects of challenge appraisal at time 1 on psychological adjustment over time ................................ 96

Figure 4.2. The analytical informational style at time 1 enhanced the positive effects of challenge appraisal at time 1 on psychological adjustment over time ............................... 96

Figure 4.3. The diffuse-avoidant style at time 1 dampened the positive effects of challenge appraisal at time 1 on psychological adjustment over time............................................ 97

Figure 4.4. The normative style to the country of origin at time 1 dampened the positive effects of challenge appraisal at time 1 on psychological adjustment over time.................. 97 


\begin{abstract}
Author notes
Chapters 2, 3, and 4 of this thesis report empirical research on acculturation and identity. These chapters were written with the intention to each be submitted for publication.

Consequently, each chapter is presented as a standalone research paper with its own introduction, method section, results, and discussion.

The research reported in this thesis was funded through the Victoria Doctoral Scholarship awarded by Victoria University of Wellington and held by the author (20122015).
\end{abstract}




\section{Chapter 1}

General introduction 


\section{General Introduction}

"Any serious and systematic attempt to study human behavior and experience must, in the very nature of things, be both developmental in depth and cross-cultural in breadth"

(Heron \& Kroger, 1981, p. 1)

\section{Migration and Changing Demographics in the Contemporary World}

Multicultural transformation of societies has evolved into a worldwide phenomenon and a pressing issue in terms of economic growth and social progress. Societies have become increasingly diverse over the past 25 years, and the immigrant population continues to rise with more than 232 million people currently living outside their countries of origin (OECD, 2013). Although recent financial and economic crises have slowed down the pace of international population moves, the size of the migrant population substantially grew in most countries between 2000 and 2010 (OECD, 2013). According to recent statistics, more than $50 \%$ of all international immigrants reside in only ten countries (i.e., United States of America, Russian Federation, Germany, Saudi Arabia, United Arab Emirates, United Kingdom, France, Canada, Australia, and Spain) in the developed world, where migration has become the primary source of population growth. Furthermore, it is projected that by 20402050 immigrants will provide the only basis for population increase in developed regions, which highlights the growing significance of migration not only in the economic prosperity of major receiving countries, but also in terms of sustainable social development (United Nations, 2013).

The report by the Commission on the Measurement of Economic Performance and Social Progress indicates that economic growth alone is not a sufficient indicator of social progress, and that social scientists need to shift their emphasis to population well-being with a particular focus on key social groups, including ethnic minorities and immigrants (Stiglitz, Sen, \& Fitoussi, 2009). Furthermore, the most recent World Migration Report empirically demonstrates the significance of immigrant wellbeing in the prediction of economic and social development (Lackzo \& Appave, 2014, p. 31).

In summary, the rapidly changing demographics along with recent studies on wellbeing and social progress underpin the importance of not only understanding the immigrant experience, but also advancing pathways to the wellbeing of migrant populations to enhance and facilitate social prosperity. This thesis aims to unpack the experience of recent immigrants by identifying the mechanisms underlying the development of a strong, coherent, 
consolidated and healthy immigrant identity, and ultimately unveiling how identity processes can contribute to the psychosocial wellbeing of immigrants over time.

\section{Acculturation Theory and Research}

\section{Acculturation and cross-cultural adaptation}

Due to the demographic expansion and diversification of societies in the Western world, intercultural contact has reached unprecedented heights. Workplaces, classrooms, and neighbourhoods are becoming ethnically more diverse as people from various cultural backgrounds come into contact on a daily basis across all life domains. Frequent and regular interactions between members of cultural groups elicit changes on both the individual and group levels, thus presenting important psychological and social implications. The process of cultural, social and psychological change occurring as the result of continuous and first-hand contact between members of different ethno-cultural groups is termed acculturation (Berry, 2005; Redfield, Linton, \& Herskovits, 1936).

Acculturation is inherently bidirectional, such that the contribution of members of the dominant/majority culture to the process of change is just as significant as the role of the acculturating individuals/groups. Consequently, changes as a result of acculturation take place on multiple levels (Berry, 1990). For example, on the group level it can involve shifts in cultural practices, social structure and institutions. For individuals, acculturation implies changes in one's behavioural repertoire, values, norms, and self-identification. The term psychological acculturation was first used by Graves (1967) to refer to all forms of alterations occurring in an individual as a result of intercultural contact.

The acculturation process involves a range of psychological (e.g., stress), social (e.g., perceived discrimination), and cultural (e.g., unfamiliar cultural practices) challenges. In a long-term context, the negotiation of these internal and external demands of transition leads to cross-cultural adaptation in one form or other. When obstacles stemming from the acculturation process are successfully overcome, positive adaptation is achieved. In contrast, when acculturating individuals fail to effectively cope with the demands of the host environment, they experience poor adaptation outcomes. Adaptation, however, can be understood and assessed in a number of ways, including health outcomes, psychological wellbeing, social effectiveness, and cultural competence (Berry, 2006).

Searle and Ward (1990) were the first to make a clear distinction between two types of adaptation domains in the context of cultural transition: psychological and sociocultural. Psychological adaptation refers to the emotional wellbeing of the acculturating person and is mainly associated with processes involved in stress management. Sociocultural adaptation, on 
the other hand, accentuates the importance of changes in one's behavioural repertoire during the acculturation process. Acquiring and utilizing culturally appropriate skills and knowledge are key factors for effective social functioning in new cultural environments. More recently, Ward, Bochner, and Furnham (2001) proposed social cognition as another guiding aspect of adaptation, along with affect and behaviour. Social cognition involves changes in cultural and social identification, social group membership, and intercultural relations. It is argued that in order to achieve favourable adaptation outcomes, it is not enough to maintain psychological wellbeing and attain sociocultural competence, but individuals in transition also need to develop a clear sense of self and obtain positive interpersonal relations. They refer to their comprehensive framework as the $\mathrm{ABCs}$ of acculturation, and recommend three main theoretical perspectives that can be used to investigate the affective, behavioural, and cognitive mechanisms underlying cross-cultural adaptation: stress and coping theory (Lazarus \& Folkman, 1984), cultural learning approach (Argyle, 1969), and models of social identity (e.g., Tajfel, 1978; Turner, Hogg, Oakes, \& Reicher, 1987) and intergroup relations (e.g., Stephan, Ybarra, \& Bachman, 1999) (Figure 1).

Figure 1.1. The ABC model of acculturation

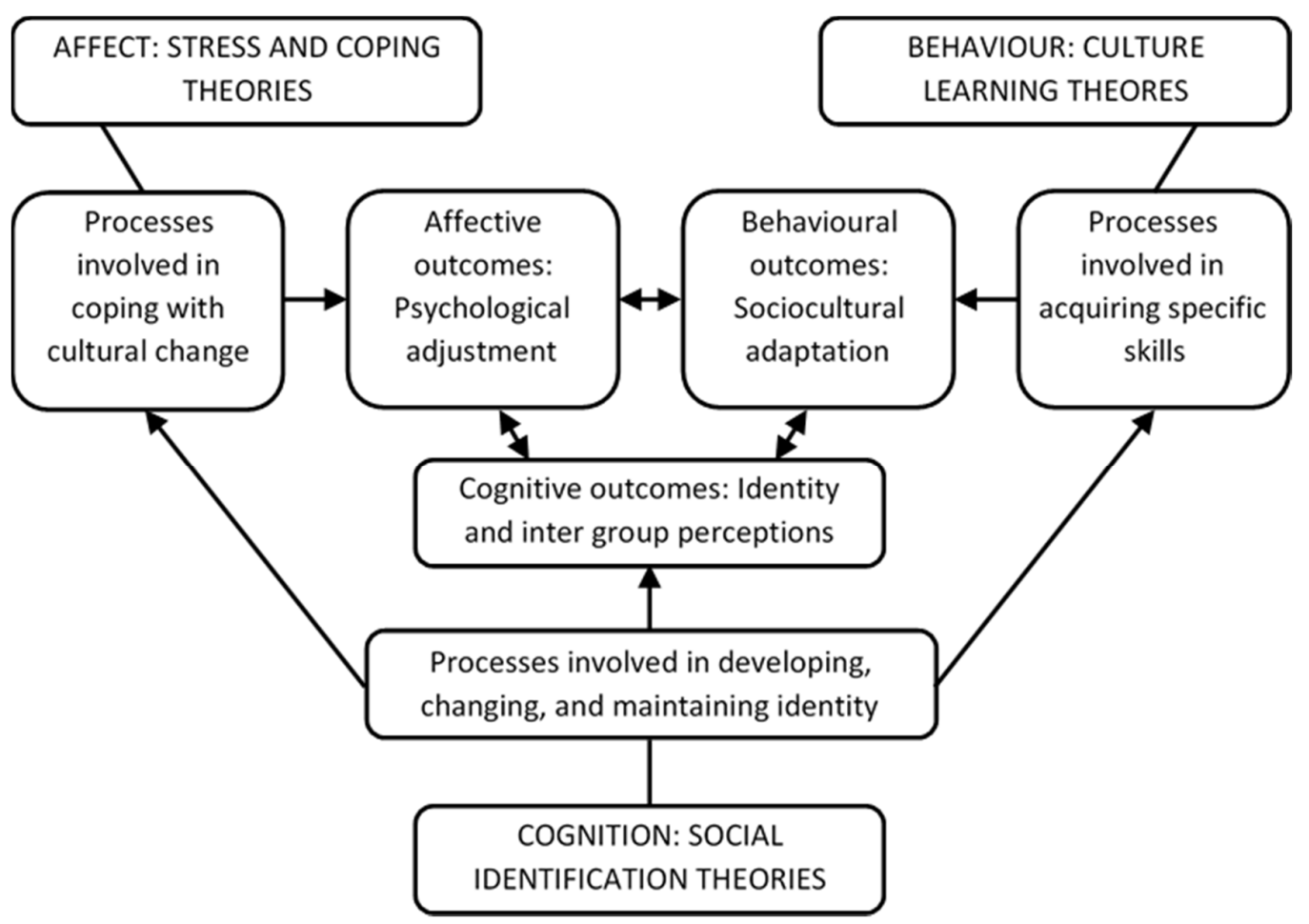


However, the ABC model - and acculturation research in general - has largely neglected developmental perspectives in understanding the acculturation experience, which has resulted in a comparative lack of longitudinal and process-oriented research projects in the field of cross-cultural psychology. The current research programme investigates psychological acculturation, i.e., processes involved on the individual level, but doing so without losing sight of the multi-layered nature of acculturation and the influence of contextual and group factors. Particular attention will be focused on the mechanisms underlying immigrants' identity reconstruction, the strategies used to develop a consolidated identity during cultural transition, and the impact of identity processes on the psychological adaptation of recent immigrants.

In the following section, I will provide a review of contemporary research on acculturation and identity by highlighting theories in connection with acculturation strategies and ethno-cultural identity integration. I end the section by evaluating the present standing of acculturation research and by drawing attention to the potential of developmental theory to contribute to our understanding of identity during acculturation. In the following section, a brief overview is provided on the psychosocial theory of identity development (Erikson, 1968) and its theoretical expansions by Marcia (1966) and Berzonsky (1989). This is followed by a summary of research on ethnic identity development of minority youth. In the subsequent section, an alternative perspective to the study of early acculturation is introduced with a particular emphasis on immigrant identity and immigrant commitment. Finally, the research programme of the current thesis is presented.

\section{Acculturation and identity}

\section{Acculturation strategies}

Although there is substantial variability in the way people approach and engage in acculturation, broader stylistic features and patterns of acculturation strategies can be identified. The most widely used framework of acculturation by Berry (2005) proposes two principal concerns that immigrants have to face during cultural transition: 1) To what extent can I / do I want to maintain the values, rules, and traditions of my original culture? and 2) To what extent can I / do I want to adopt the values, rules, and traditions of the dominant culture? This bi-dimensional perspective postulates that the maintenance of the original culture and the adoption of the culture of the host society are not mutually exclusive, and that identification with one culture does not necessarily imply the rejection of the other one. Based on the extent of change on each dimension and the degree of involvement in the two cultures, four potential acculturation strategies emerge. Integration occurs when one wants to 
maintain the heritage culture and at the same time is inclined to adopt and participate in the culture of the receiving country. In contrast, marginalization ensues when both cultures are rejected. High levels of involvement and participation in the new culture and low levels of maintenance of the heritage culture result in assimilation. Finally, separation is pursued when the original culture is maintained and embraced, whereas the new culture is rejected.

Assimilation involves the most extensive psychosocial changes, whereas separation requires minimal shifts in terms of behaviours, values, and identity. Integration and marginalization entail moderate levels of alterations (Berry, 2006). In general, integration is the most preferred acculturation strategy (Berry, 2005), and studies converge to link integration to better psychological outcomes in the form of lower levels of psychological symptoms and depression compared to the other three orientations (Krishnan \& Berry, 1992; Ward \& Kennedy, 1994; Ward \& Rana-Deuba, 1999).

\section{Ethnic and cultural identity integration}

In recent years considerable attention in cross-cultural psychology has been focussed on how identity constructs and processes intertwine with acculturation and cross-cultural adjustment. A substantial body of research has investigated the impact of acculturation on identity, with the main focus on changes in the ethnic and cultural aspects of identity. Changes in ethnic and cultural self-identification involve similar processes as those described in acculturation strategies (Liebkind, 2006; Phinney, 1990). Specifically, acculturating individuals show large variations in their motivations to maintain their ethnic identity and to identify with the broader national culture. However, cultural orientation and cultural identification should not be equated, as one can have a strong orientation to adopt elements and features of a given culture without necessarily identifying with it (Liebkind, 2006; Snauwaert, Soenens, Vanbeselaere, \& Boen, 2003; Ward \& Kus, 2012), which might often be the case for newly arrived immigrants.

Even though Berry's bidimensional model of acculturation strategies was first conceptualized mainly in terms of attitudes and behaviours, the theory has been later refined to incorporate value orientations as well as identity dimensions (Berry, 2009). Studies have been undertaken to assess orthogonal cultural identification in the framework of the bidimensional model (Oetting \& Beauvais, 1991; Sayegh \& Lasry, 1993) and the associations between acculturation strategies and identity (Berry, Phinney, Sam, \& Vedder, 2006; Ryder, Alden, \& Paulhus, 2000; A. L. Schwartz, Galliher, \& Rodriguez, 2011; Ward, 2006). Based on research testing this refined model (Berry, 1990, 2009), most scales have been designed to incorporate elements of ethnic identity presuming that important changes take place in the 
identity domain (Birman, Trickett, \& Vinokurov, 2002; Chung, Kim, \& Abreu, 2004; Cuéllar, Harris, \& Jasso, 1980; Lim, Heiby, Brislin, \& Griffin, 2002; Suinn, Ahuna, \& Khoo, 1992). In sum, a growing number of studies show that acculturation has important implications for identity, as it facilitates acculturating individuals, whether they be immigrants, ethnic minorities, or members of indigenous groups, to think thoroughly about who they are, what values and goals they endorse, and how they changed as a result of contact with the members of the national mainstream (Berry et al., 2006; Ward \& Kus, 2012).

More recently, Benet-Martinez and Haritatos (2005) have introduced Bicultural Identity Integration (BII) as an over-arching framework to investigate individual differences in the structure and organization of multiple cultural identity aspects. BII assesses levels of identity integration along two dimensions: cultural harmony vs. conflict and cultural blendedness vs. distance. More specifically, those characterized by an integrated bicultural identity perceive their cultural identity aspects as being harmonious, compatible and overlapping, as opposed to being dissonant, conflicted, and separated. Studies undertaken with Chinese immigrants and international students have found that bicultural identity integration positively predicts psychological adjustment as assessed by both positive (e.g., life satisfaction) and negative indicators (e.g., depression) (Chen, Benet-Martinez, \& Bond, 2008). Although Berry and Benet-Martinez approached the acculturation-identity relationship from slightly different perspectives, the lines of research stimulated by both of their respective theories indicate that an achieved and consolidated identity promotes the most beneficial outcomes for acculturating individuals, leading to healthy and adaptive socialpsychological functioning (see A. M. D. Nguyen \& Benet-Martinez, 2013).

\section{Evaluating theory and research on acculturation and identity}

Over the last two decades, cross-cultural psychology has gained a deeper understanding of ethnic and cultural identification and the role of integration for members of acculturating groups. However, there remains a paucity of research on identity change in adult immigrants (Liebkind, 2006), especially at the early stages of cultural transition. Although theories and the empirical research evolving from this tradition aim to describe structural changes in identity, they focus solely on how ethnic and cultural identity aspects might be transformed as a result of intercultural contact. Little is known about changes in other social or personal identity aspects as a result of cultural transition, and how commitment to other identity aspects might influence the cross-cultural adaptation of recent immigrants. 
Furthermore, in the acculturation arena the majority of research conducted is crosssectional, thus providing a static representation of changes involved during cultural transition and cross-cultural adjustment. The positive consequences of integration are undisputed; however, little research has been done to investigate the processes involved in achieving integration and a consolidated identity in the context of cultural transition.

Approaching the acculturation-identity issue from a more developmental standpoint, as opposed to Berry's social and cross-cultural perspective, S. J. Schwartz, Montgomery, and Briones (2006) proposed that cultural transition inevitably affects the identity development of acculturating individuals, as changes in the socio-cultural context can cause a disequilibrium or conflict in personal identity. In addition, they strongly argued that in today's world acculturation and the identity issues faced by immigrants should be one of the primary foci of identity research. In the following section, I argue that Erikson's $(1950,1968)$ psychosocial theory of identity development, and corresponding empirical research, provide a useful framework to understand the mechanisms involved in identity reconstruction of immigrants during cultural transition.

\section{A Developmental Perspective on Identity}

\section{Identity and identity development}

Over the past 20 years the use (and abuse) of the term 'identity' has been a major concern across the social sciences, and especially in the field of psychology (Adams \& Marshall, 1996; Berzonsky \& Adams, 1999; Brubaker \& Cooper, 2000; Côté \& Levine, 1987; S. J. Schwartz, 2001, 2005; van Hoof, 1999). The inception of identity theory dates back to the 1950s, when Erikson published his first papers on the topic. Erikson (1968) defined identity as "a subjective sense of personal sameness and continuity, paired with some belief in the sameness and continuity of some shared world image" (pp. 18). He drew a clear distinction among three aspects of identity: ego, personal, and social. Ego identity refers to the most fundamental and unconscious beliefs that are temporally consistent and resistant to change. Personal identity, on the other hand, consists of values, goals, beliefs, and norms that are publicly expressed and consciously construed. Finally, social identity involves solidarity with and commitment to various social groups. Erikson's theory emphasizes the role of the broader socio-cultural context in identity formation and postulates that identity development is the result of a dynamic interplay between the person and their environment.

Although the definition of identity has been advanced by contemporary scholarship, the social perspective still deeply impregnates identity theory (see Adams \& Marshall, 1996; Cote, 1997). For example, by adopting a social-developmental point of view Adams and 
Marshall (1996, p. 433) defined identity as a self-regulatory, social-psychological construct that directs attention and influences information processes and behaviour. Identity helps us understand who we are, creates meaning and commitments, forms coherence and harmony between goals, beliefs and values, and fosters a sense of personal control and the ability to recognize future possibilities. They argued that identity is embedded in social context at both the micro and macro levels. The micro context involves one's immediate interpersonal relations, whereas the macro system represents the broader socio-cultural environment (including cultural norms, values, traditions, and social institutions), within which the individual is embedded. Changes in the social contexts shape identity and encourage people to continuously reorganize their self-definitions through differentiation and integration.

Following in the footsteps of Adams and Marshall (1996), I interpret identity as a hierarchically organized frame for self-definition that is embedded in nested levels of socialcultural contexts (see Bronfenbrenner \& Morris, 2006). Change in each level of the broader context can have important consequences in terms of identity development. Furthermore, I differentiate among four structural levels that vary in terms of abstraction (Figure 2.). On the most concrete level identity incorporates identity elements, namely the values that individuals hold, the norms and rules by which they live their lives, the beliefs they endorse, and the aspirations they pursue. Selections and combinations of identity elements merge into more abstract personal and social identity aspects. For example, some values might be more influential in defining one's cultural identity (i.e., respect for traditions), whereas other identity elements have more importance in terms of personal identity aspects (i.e., valuing creativity and thriving for indulgence). Of course, most identity elements contribute to a variety of identity aspects. Preserving the public image, for instance, can be equally important for one's personal, cultural or professional identity aspects.

On a more abstract level, the identity structure describes the connections, overlap, and permeability among different aspects of identity. This level often forms the focus of investigations in cross-cultural research. For example, Bicultural Identity Integration (BenetMartinez \& Haritatos, 2005) captures the structural relationships between two (or more) cultural-identity aspects. According to the BII framework, bicultural individuals can either integrate the aspects of the multiple cultures into a blended structure or keep them compartmentalized. Importantly, however, the identity structure does not tell us whether one has achieved a consolidated identity or experienced identity conflict. Keeping identity aspects separated or impermeable does not necessarily result in identity confusion, conflict or negative self-view. Similarly, a blended identity does not automatically indicate identity 
consolidation, even though studies converge to link integration to more positive outcomes (Berry et al., 2006; Chen et al., 2008).

In my terminology, a consolidated identity is achieved when the individual demonstrates positive identity outcomes. Specifically, identity outcomes refer to the evaluation (i.e., affective component) and perception (i.e., cognitive component) of the identity as a whole. For example, I argue that a person who has a consistent sense of self and a clear perception of how his or her identity aspects are interrelated, and more importantly, feels satisfied and positive about the overall organization and content of his or her identity, will be characterized by a balanced/achieved/consolidated identity. Identity outcomes can be assessed by constructs including, but not limited to, self-esteem, self-concept clarity, identity balance, identity conflict, or identity confusion. My view on identity outcomes was inspired by Schwartz et al.'s (2006) work, which argued that an adaptive identity requires coherent and consistent personal and social identity aspects.

Finally, the term identity processes is used to summarize the range of mechanisms, strategies, and practices that promote and guide identity development. According to Erikson (1968), identity development is a central task for all individuals. The reconstruction of identity is facilitated by experiencing disequilibrium in one's identity, which generally results in an identity crisis (Schwartz, Klimstra, Luyckx, Hale, \& Meeus, 2012). Disequilibrium ensues when the individual confronts conflicting internal and/or external demands. However, identity crises are normative and expected over the course of the lifespan and function as antecedents of the transition from one developmental stage to another. Thus crises are not necessarily periods of breakdown and threat, but they are sensitive phases of individual development that provide the potential to grow and achieve a new identity (Luyckx, Klimstra, Duriez, Van Petegem, \& Beyers, 2013). The reconstructed identity will still have connections and forms a continuity with the previous identity, but it is expanded to cope with new beliefs, roles and commitments.

Marcia $(1966,1988)$ proposed two independent dimensions as the main pillars of identity development: exploration and commitment. Exploration refers to a set of behaviours applied in response to the tasks involved in creating, maintaining or reconstructing one's identity. During exploration people seek information, examine and consider alternative options. Commitment, on the other hand, requires decisiveness in terms of goals, clarity about values, and adherence to beliefs and ideas. Marcia argued that the two dimensions are equally important and functional in terms of identity development. Specifically, people need to actively explore possible alternatives and also be willing to commit themselves to specific 
values and ideals in order to develop an achieved identity. Depending on the level of engagement in both dimensions, people can be characterized by an achieved identity (high exploration and high commitment), a foreclosed identity (low exploration and high commitment), moratorium (high exploration and low commitment), or a diffuse identity (low exploration and low commitment).

Although Marcia identified the underlying components of identity development and described possible outcomes in forms of identity status, his theory has been criticized on multiple grounds. Many have argued that the identity status paradigm under-represents Erikson's concept of identity, and that it works better as a character typology as opposed to a developmental stage model, since it does not say much about the process of reconstruction, i.e. how and why individuals become identity achievers (see the review by Schwartz, 2001).

Figure 1.2. Hierarchical model of identity

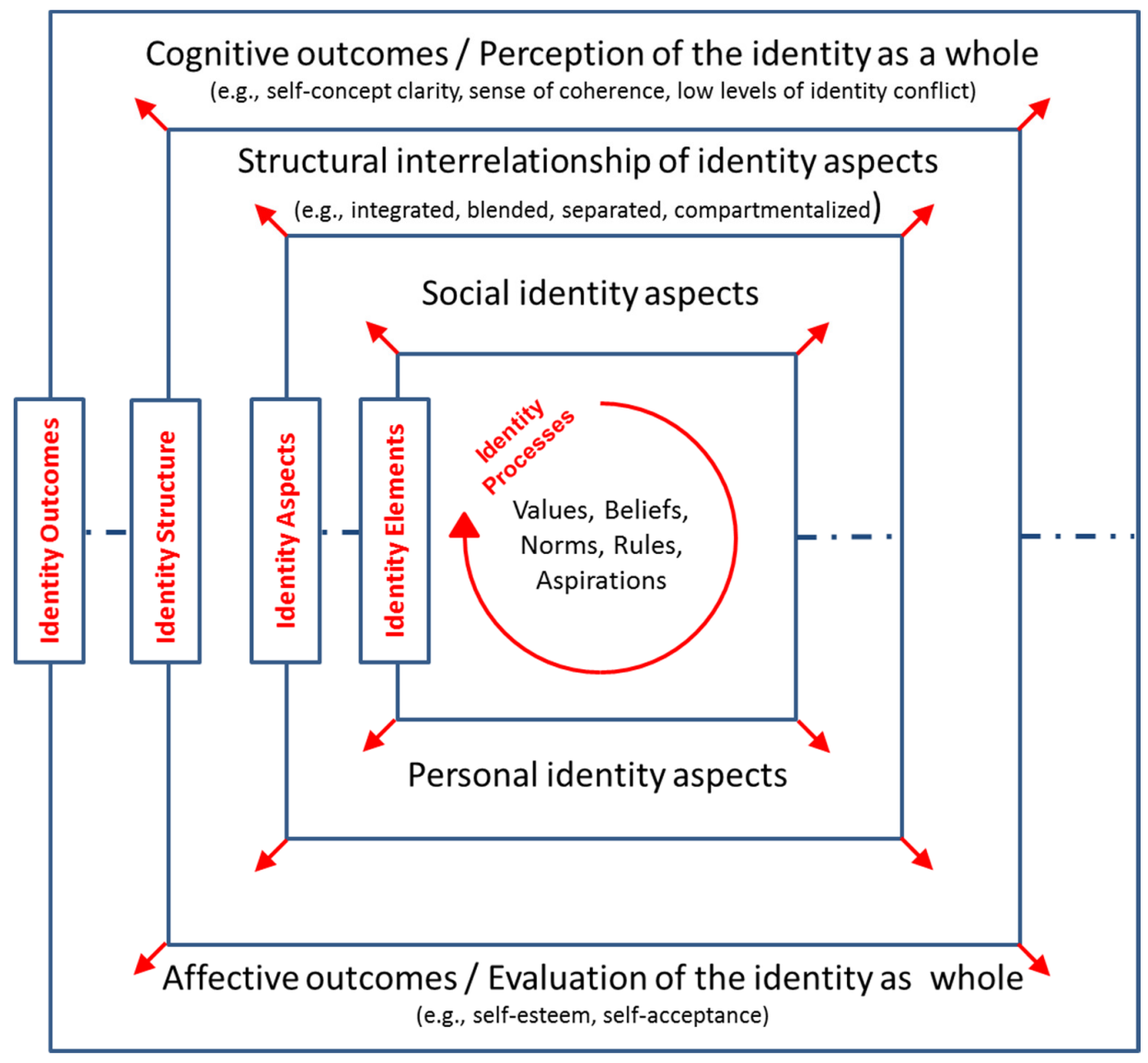




\section{Social-cognitive theory of identity styles}

Marcia's work has, however, stimulated a large body of research and theorizing that are commonly referred to as the Neo-Eriksonian theories. One of the many significant extensions of Marcia's work was introduced by Berzonsky (1989), who aimed to identify the driving mechanisms of identity exploration and answer the urgent questions of how and by what means people achieve a new identity. Building on Marcia's (1966) identity status paradigm, Berzonsky developed a process-oriented approach to identity development to pinpoint and describe the underlying mechanisms of exploratory behaviour. According to the social-cognitive theory of identity styles, relatively stable individual differences can be observed in the ways people approach identity stress, examine identity-relevant issues (e.g., values, norms, and commitments), process information, and make decisions during identity exploration (Berzonsky, 1989, 2011).

By extrapolating Marcia's theory (1966) with a particular focus on the dimension of exploration, Berzonsky $(1989,2011)$ proposed three distinct information processing and decision making strategies that underpin identity development: informational, normative, and diffuse-avoidant styles. The informational style implicates active engagement in selfexploration, information seeking and openness to self-relevant feedback. Those who rely on informational processing generally feel challenged, as opposed to threatened, when they experience differences between their own views and the views of significant others or reference groups. In contrast, people who show a preference for the normative style experience high levels of anxiety, worry and frustration if their values and norms do not overlap with the values and norms represented by their significant others and/or the society in which they live. In order to reduce feelings of discomfort, they automatically internalize and adopt the values, rules and behaviours that are perceived to be socially desirable. Finally, a diffuse-avoidant orientation entails strategic avoidance of self-relevant feedback and confrontation. Those with a diffuse-avoidant style are present oriented and motivated by situational demands and social factors, such as popularity and reputation.

Twenty-five years of extensive research has followed providing clear and convincing evidence for the existence of the three styles and their socio-cognitive (Berzonsky, 2008; Berzonsky et al., 2013; Berzonsky \& Sullivan, 1992; Crocetti, Rubini, Berzonsky, \& Meeus, 2009; Smits, 2009; Soenens, Duriez, \& Goossens, 2005; Watson \& Morris, 2005), behavioural (Adams, Munro, Munro, Doherty-Poirer, \& Edwards, 2005), and personality (Dollinger, 1995; Duriez \& Soenens, 2006) correlates. In addition to establishing the nomological network of identity styles, a substantial body of research has been undertaken to 
demonstrate the role of social-cognitive identity styles in the prediction of identity statuses and outcomes.

An informational style is generally related to high levels of exploration, strong commitments and an achieved identity (Berman, Schwartz, Kurtines, \& Berman, 2001; Berzonsky, 1992a, 2008; Berzonsky \& Kuk, 2000; Berzonsky et al., 2013; Krettenauer, 2005). People with an informational orientation are inclined to engage in identity revision and are characterized by a well-differentiated and well-integrated identity structure that is anchored in personal self-esteem (Berzonsky, Macek, \& Nurmi, 2003). The normative dimension has been associated with increased levels of commitment but minimal exploration (Crocetti et al., 2009), thus ultimately resulting in a foreclosed identity (Berman et al., 2001; Berzonsky, 1992a, 2008; Berzonsky \& Kuk, 2000; Berzonsky et al., 2013; Krettenauer, 2005). It is important to highlight that a normative orientation can also lead to an achieved identity that is, however, anchored in collective self-esteem (Berzonsky et al., 2003). Those who process on a diffuse and avoidant basis have weak commitments and do not show much interest in exploration. The style has been consistently linked to a loosely integrated and present-oriented identity structure (Berzonsky et al., 2003) and a diffuse identity (Berzonsky, 1989).

The main premise of Berzonsky's theory is that every individual has the cognitive capacity to apply all three social-cognitive styles; however, people generally embody a dominant orientation, a strategy they consistently apply in response to identity stress throughout the lifespan and across situations (Berzonsky \& Ferrari, 1996; Berzonsky \& Kuk, 2005). I believe that the social-cognitive perspective on identity development provides an effective framework to study acculturation identity crises and to advance our understanding of the processes involved in creating a coherent immigrant identity.

\section{Identity development in acculturating individuals/minority groups}

Phinney (1989) was one of the first scholars who attempted to provide an analysis of changes in identity during acculturation by drawing on social identity theory (Tajfel, 1978; Tajfel \& Turner, 1986) and adapting Marcia's $(1966,1988)$ identity stratus paradigm to the ethnic identity development of minority adolescents in the United States. The Multigroup Ethnic Identity Measure was developed to assess two components of ethnic identity formation: exploration and commitment (Phinney, 1992; Phinney \& Ong, 2007). Exploration involves participation in the ethnic community, seeking information and acquiring knowledge about one's cultural heritage. Commitment, on the other hand, refers to an affirmation of 
group membership, a sense of clarity and positive feelings about belonging to a particular ethnic community.

Studies have consistently shown that a strong and achieved ethnic identity predicts positive psychosocial functioning among ethnic youth (Dimitrova, Bender, Chasiotis, \& van de Vijver, 2013; Roberts et al., 1999; Umana-Taylor \& Updegraff, 2007); however, this association is assumed to be more robust when identification with the heritage culture is paired with a strong orientation to the mainstream culture (Berry et al., 2006; Phinney, 1991). Phinney's work has provided important insights into the psychosocial correlates and the role of ethnic and national identification during the acculturation process. Furthermore, it has inspired a large body of investigations on ethnic and national identity development in immigrant and ethnic minority youth.

In recent years, particular attention has been focused on three particular domains in the field of ethnic identity research. First, researchers point to the urgent need for understanding the longitudinal trajectories of ethnic identity development beyond adolescence. A study conducted by Syed and Azmitia (2009) with a diverse sample of college students in the United States examined the ethnic identity development over a three-year long period. Testing both individual and group-level differences, their findings revealed that exploration and commitment consistently increased during the college years across ethnic groups. Although they found no significant ethnic differences in ethnic identity development over time, immigrant and ethnic minority youth reported significantly higher levels of initial exploration and commitment. In the individual level, personal identity resolution (i.e., general identity clarity and satisfaction with the self) was related to both exploration and commitment across time. These findings suggest that identity development does not terminate at the end of adolescence, but rather it continues during emerging adulthood and is likely to be a life-long project (Fadjukoff, Pulkkinen, \& Kokko, 2005). Furthermore, Syed and Azmitia argued that the longitudinal relationship between ethnic identity, general identity, and self-esteem provides further confirmation for Erikson's (1968) theory of an integrated self-identity.

Secondly, there has been growing interest in the study of ethnic identity exploration. Although substantial research has been done to investigate the relationship between ethnic identity exploration and wellbeing, findings vary as a function of the measures used. More specifically, studies using the Ethnic Identity Scale reported positive associations between exploration and wellbeing (e.g., Umana-Taylor \& Updegraff, 2007). By contrast, studies assessing exploration with the MEIM found no significant relationships between the two constructs (e.g., Ghavami, Fingerhut, Peplau, Grant, \& Wittig, 2011). In order to identify the 
source(s) of these inconsistencies, a collaborative research project was carried out in the United States with more than 3,600 ethnic minority youth (Syed et al., 2013). Two main dimensions of ethnic identity exploration emerged: participation and search. Higher scores on participation indicate active involvement in the ethnic community, whereas search refers to information seeking and information processing in relation to one's cultural group. Results revealed that participation positively predicted identity coherence and was negatively related to identity confusion. In contrast, ethnic identity search was linked to increased levels of identity confusion. Although neither participation nor search had a direct impact on psychological adjustment, they exerted indirect effects on wellbeing through identity confusion and coherence. These findings highlight that identity exploration is multidimensional and that different forms of exploration contribute to different pathways to wellbeing.

Finally, a different line of research has evolved to gain a deeper insight into specific forms of identity commitment. Umaña-Taylor, Yazedjian, and Bámaca-Gómez (2004) have proposed a three-component model of ethnic identity including identity exploration, identity resolution, and identity affirmation. According to their framework, it is important to make a clear distinction between the cognitive (i.e., resolution) and affective (i.e., affirmation) components of ethnic identity. Umaña-Taylor and colleagues argued that the MEIM paradigm implies that commitment to an ethnic identity is related to positive feelings toward the ethnic group. However, having a clear sense of self in terms of one's ethnic identity (i.e., identity resolution) does not necessarily mean that the individual associates positive affect with that resolution (i.e., identity affirmation). In fact, it has been shown that affirmation is generally unrelated to self-esteem and familial ethnic socialization, whereas identity resolution is positively related to both constructs (Umaña-Taylor, Gonzales-Backen, \& Guimond, 2009). However, further studies have suggested substantial ethnic variations in the relationships between the three components of ethnic identity and self-esteem (Umaña-Taylor \& Shin, 2007). In addition, longitudinal findings have indicated that both affirmation and resolution increase from middle to late adolescence in Latina girls, but only affirmation continues to rise in Latino boys (Umaña-Taylor et al., 2009). The three lines of research discussed above call attention to the value of longitudinal studies in identity research, the importance of understanding the processes involved in exploration, and the key role of identity commitment in developing a consolidated identity and advancing wellbeing.

Much less research has been done to investigate differences across acculturating groups in identity processing styles underlying exploration behaviour. One of the few such 
studies conducted has revealed that Black and Hispanic university students scored significantly higher on the normative style compared to their non-Hispanic White peers (S. J. Schwartz, Cote, \& Arnett, 2005). In another investigation undertaken with immigrant youth, S. J. Schwartz and Montgomery (2002) found that second generation immigrants were less likely to use informational strategies compared to their first and third generational counterparts. However, there was no difference between the first and third generation in the preference for the informational style. Although these studies examined identity processes in acculturating groups, including both immigrant and ethnic minority youth, the focus of both investigations was on normative identity development, such that they aimed to identify the strategies used by members of different ethnic groups to address a developmentally induced identity task and develop positive personal identity outcomes. However, for recent immigrants, who have decided to change cultures as adults, identity reconstruction during acculturation is related to the struggle to develop commitments in the context of the receiving society. In this case identity crises are evoked by situational-contextual, as opposed to developmental, changes.

\section{Immigrant Identity: An Alternative Perspective}

\section{Identity as an immigrant}

Moving to a new culture involves a number of immediate changes in terms of one's social position, group memberships, and legal status. For example, as soon as an individual crosses borders with the intention to stay in the country for a longer period of time, he or she is considered to be an international immigrant, thus inevitably becoming a member of a new social group. During the early periods of cultural transition, intragroup (e.g., with other immigrants) and intergroup (e.g., with host nationals) contact increase rapidly; therefore, both social and personal identity aspects become particularly salient (Sussman, 2000; Turner, Oakes, Haslam, \& Mcgarty, 1994).

Host nationals perceive and treat newcomers as immigrants whether they identify with this social category or not. Each day they are faced with questions, such as "Where are you from?", "How do you do things in your culture?", "Is it only you that behaves like this or is it also other people in your culture?", "How long have you been here and how long are you going to be here for?", "Do you find it very different here compared to your home country?". For someone, who has not lived abroad before, this might be the first time that they become aware of their cultural heritage and start to think about their commitments, the values they endorse, or the rules and traditions they want to follow. The frequency of having to answer these types of questions might reduce over time, but at the early stages of transition 
immigrants are constantly reminded that they do not originate from the host country and are primed to think about who they really are in terms of values, commitments, aspirations, and norms.

In general, socio-cultural expectations drive the individual in transition to seek information about the new environment and the rules of the society of settlement, to examine the values they hold, to explore alternative options in the new culture, to adopt a legal and social status, and to reconstruct his or her identity as an immigrant. Furthermore, most immigrants have to engage into this intense exploration process using a foreign language, which can greatly limit one's ability to express their true personality and present themselves in a manner that is authentic and engaging (Pellegrino Aveni, 2005). Ultimately, being part of this new social category has psychological, social, and legal implications.

In conclusion, I propose that the trigger for personal identity exploration and reconstruction is not cultural transition per se, but is instead being ascribed to a particular social category post transition. There are other groups of culture crossers, such as tourists, who might spend months in a new culture without undergoing major identity exploration or acculturation identity crisis. I believe that there is merit in investigating the immigrant identity for two main reasons. Although it is an ascribed social category based on legal status, the immigrant identity might develop in to a new social identity aspect, i.e., people can identify as immigrants even when they are not considered to be immigrants in legal terms anymore. Moreover, commitment to an immigrant identity does not require the reconciliation of ethnic and national identity aspects, but it involves changes in terms of personal identity aspects. Consequently, it can be a particularly meaningful framework for the study of newcomers or people who do not immigrate to acculturate (see Boski, 2013).

\section{Applying developmental theory to immigrant identity}

One of the primary goals of human development is for people to endorse values, adopt behaviours, and develop commitments in order to be effective in their socio-cultural environments. However, moving to a different cultural context changes the frame of social reference and presents an alternative social reality (Chirkov, 2009). Immigrants generally experience a considerable degree of dissimilarity (i.e., cultural distance) between the heritage and the host culture in terms of values, rules, norms, and socially accepted behaviours. Previous research has shown that experiencing differences between the former and the new social context influences social interactions (Galchenko \& van de Vijver, 2007), crosscultural adaptation (Ward \& Searle, 1991), and the ways immigrants reconstruct or maintain 
their identity structure, identify themselves with specific values, and create new commitments (Adams \& Marshall, 1996; Konig, 2009; Ryder et al., 2000).

Changes in the socio-cultural context can give rise to an identity crisis or conflict (Baumeister, Shapiro, \& Tice, 1985). Identity conflicts occur when a new identity aspect has to be integrated into the self that is largely incompatible with existing self-definitions. This can result in the experience of stress, negative feelings, and conflict over the identity. In this case the conflict is related to adjustment difficulties and can be observed when people enter into a system representing new values and norms. In a series of three studies, Ward, Stuart, and Kus (2011) demonstrated that identity conflict is positively associated with identity distress, depression, psychological symptoms, perceived discrimination, and socio-cultural adaptation problems. A negative relationship was found with self-concept clarity, sense of coherence, and harmony. Furthermore, those who preferred integration as an acculturation strategy reported less ethno-cultural identity conflict compared to their separated, marginalized and assimilated peers.

In sum, Ward et al.'s (2011) findings indicated that experiencing a conflict over one's identity during cultural transition can have harmful effects in terms of psychological and sociocultural adjustment. Conversely, a large number of empirical studies has provided evidence for the benefits of positive personal identity outcomes, including self-concept clarity, sense of coherence and self-esteem (Aycan \& Berry, 1996; Costigan, Koryzma, Hua, \& Chance, 2010; Jasinskaja-Lahti \& Liebkind, 2001; H. H. Nguyen, Messe, \& Stollak, 1999; Safdar, Lewis, \& Daneshpour, 2006; Usborne \& Taylor, 2010; Ying, Lee, \& Tsai, 2007) in connection with the psychological adjustment of immigrants. These findings support Schwartz et al.'s (2006) contention that a consolidated and strong personal identity can greatly facilitate the adjustment process, whereas a conflicted, unbalanced and fragmented identity hinders adaptation in the context of cultural transition.

Notwithstanding that the investigations discussed above provide a comprehensive insight into the role of positive and negative identity outcomes in the context of cultural transition, they tell us little about the mechanisms underlying the identity reconstruction of immigrants. Immigrants encounter a wide range of difficulties on a daily basis, including barriers to effective communication, discrimination, financial problems, and clashing values just to name a few. It is important to recognize that identity outcomes, such as self-esteem and self-concept clarity, can function as protective factors during periods of life predominated by acculturative demands and obstacles. At the same time, however, if we want to fully comprehend the immigrant experience, it is just as crucial to gain an understanding of how and 
by what means people in transition can maintain high levels of self-esteem or achieve a clear and consolidated identity in spite of the daily challenges of acculturation.

One approach that, I believe, could greatly benefit research on immigrant identity is developmental theory. In the field of developmental psychology (see section 3 in this chapter), strategies and processes involved in overcoming normative identity crises and creating an achieved identity have been intensively and widely researched. Moreover, Berzonsky (2008) has argued that stylistic differences in approaching identity stress are a result of individual preferences as opposed to different developmental paths. Stylistic orientations are relatively stable over time and show little fluctuation, which suggests that individuals utilize the same, habitual strategies across time and situations in order to overcome identity crises. I argue throughout this thesis that acculturation identity crises are normative periods of cultural transition that involve similar processes and demands (i.e., exploration behaviour and commitment) as what have been considered in connection with developmentally evoked identity crises. Consequently, it is proposed that social-cognitive identity styles are in operation during acculturation identity crises underlying the identity reconstruction of recent immigrants. More specifically, they contribute to both positive and negative identity outcomes and have long-term impact on the psychological wellbeing and cross-cultural adjustment of immigrants.

Chirkov (2007) has argued that "the goal of the cultural psychology of acculturation is to describe and understand the change of the constitutive rules of immigrants' multiple cultural realities and to understand changes, problems, conflicts, that immigrants undergo because of differences in these two realities and to understand meanings and dynamics of their actions as they try to negotiate through these realities" (p. 90, italics added by the authors). Integrating developmental theory into the existing frameworks of acculturation research, such as the $\mathrm{ABC}$ model, gives us an unparalleled opportunity to understand acculturation identity processes by investigating social-cognitive information processing styles in the context of cultural transition, to study change over time by adopting longitudinal research methods, and to gain insights into the dynamics of the immigrant experience through the examination of commitment to an immigrant identity, as opposed to merely assessing levels of ethnic and cultural identity aspects. In summary, with the current research program I aim to offer a novel perspective on how identity may be examined in mobile, acculturating individuals.

\section{Present Research Program}

The current research program aims to provide an extensive examination of the identity processes underpinning the identity development and psychological adjustment of 
immigrants. It is important to emphasize that the present investigation is less concerned with the explicit reconciliation of ethnic and national identity aspects (i.e., structural integration) and is more interested in gaining a deeper insight into the development of a committed, consolidated, and positively evaluated identity as an immigrant. Therefore, the studies presented in the thesis do not assess ethnic, cultural or national identity in any form. Instead, an in-depth analysis of identity outcomes are presented with diverse samples of immigrants.

Integrating elements of cross-cultural and developmental theory, the series of studies reported in the present thesis investigate three central themes of early acculturation: 1) the variety of social-cognitive identity strategies operating in an acculturation identity crisis, 2) the mechanisms underlying the development of a positively evaluated immigrant identity, and 3 ) the role of identity processing styles in acculturative stress management and the prediction of psychological adjustment.

Chapter 2 reports three independent empirical studies that were conducted with the main goal of developing a version of the Identity Style Inventory (Berzonsky, 1989) that is capable of effectively measuring social-cognitive identity processing strategies and identity commitment during cultural transition. The Identity Styles Inventory - Cultural Transition Version (ISI-CT) is introduced in this chapter and the psychometric properties of the measure are analysed. The first study describes the scale adaptation process and tests the factor structure, and the convergent and divergent validity of the new assessment tool. Furthermore, relationships between demographic variables and the ISI-CT subscales are examined. The second study aims to confirm the factor structure of the measure and to provide further information on group differences based on gender, ethnic visibility, and previous sojourner experience. Finally, the third study investigates longitudinal relationships among the subscales using cross-lagged analysis.

In chapter 3, building on the framework of psychosocial development (Erikson, 1968), a model of immigrant identity development is proposed. The theoretically-driven model is probed with a relatively large sample of recent immigrants using structural equation modelling. This chapter aims to address the second research question to provide a deeper insight into the dynamics of identity reconstruction during cultural transition. In particular, identity commitment is predicted to mediate the relationships between identity processing styles and identity outcomes. Both direct and indirect paths are examined and an alternative model is presented, in which commitment functions as a moderator, as opposed to a mediator. Findings highlight both positive and negative pathways to a consolidated immigrant identity, 
draw attention on the key role of commitment in identity development, and provide further support for the functionality of identity styles in predicting favourable identity outcomes.

Drawing on the stress and coping framework, Chapter 4 scrutinizes the role of identity processing styles in acculturative stress management and the prediction of psychological wellbeing. A 6-month longitudinal study is reported assessing two sets of predictions. First, the main effects of challenge and threat appraisals to personal identity are examined on psychological adjustment over time. Next, interaction effects between identity processing styles and stress appraisals are investigated in order to understand whether (and if yes, how) social-cognitive strategies can buffer the negative impact, or enhance the positive effects, of appraisal on the psychological wellbeing of immigrants six months later.

The final chapter of this thesis (Chapter 5) is set out to synthetize the outcomes of the empirical studies presented in the second, third, and fourth chapters. A comprehensive summary is provided and the main findings discussed within the realms of acculturation and developmental literature. The strengths and limitations of the research programme, along with avenues for future research, are reviewed and critically examined. Furthermore, special attention is focused on the potential application domains and the implications of the results for not only the immigrant population but also the settlement societies. 


\begin{abstract}
Author note
Three studies were conducted to test the research questions described in Chapter 1. Study 1 involved immigrants residing in New Zealand, and the data were used for scale development and validation (Chapter 2). Study 2 was conducted with a larger sample of immigrants and served two main purposes. First, data from the total sample were used to confirm the factor structure of the Identity Style Inventory - Cultural Transition Version (Chapter 2). Second, a subsample of newly arrived immigrants was separated from the total sample and used to test a model of identity development (Chapter 3). Study 3 was a longitudinal investigation of identity and adaptation. Longitudinal relationships among the ISI-CT subscales were calculated to examine the temporal stability of the styles. These findings are reported in Chapter 2. In addition, longitudinal moderation analyses were employed to elucidate the role of identity processing styles in identity stress management and the prediction of psychological adaptation over time (Chapter 4).
\end{abstract}




\title{
Chapter 2
}

\section{Identity processing styles during cultural transition: Construct and measurement}

\author{
Agnes Szabo, Colleen Ward, \& Garth J. O. Fletcher \\ Victoria University of Wellington
}

Manuscript submitted 


\begin{abstract}
Drawing from previous work on identity styles by Berzonsky $(1989,2011)$, the current paper introduces a new version of the Identity Style Inventory assessing social-cognitive information processing strategies during cultural transition (ISI-CT). The ISI-CT incorporates five distinct identity styles (analytical informational, exploratory informational, normative to the country of origin, normative to the host society, and diffuse-avoidant) and a measure of identity commitment. The paper reports the construction of the ISI-CT and examines its factor structure (study 1 and study 2), convergent and discriminant validity (study 1 ), and longitudinal relationships among subscales (study 3). Findings support reliability and validity of the ISI-CT as an assessment of the identity processes underlying identity development of people in cultural transition.
\end{abstract}




\section{Introduction}

More than 230 million people live outside of their countries of origin, and this number is continuously rising (OECD, 2013). Intercultural contact has become an everyday experience for a large number of people, and the fact that half of all international migrants are residing in only ten countries highlights the growing socio-cultural significance of this group and calls attention to the importance of understanding the dynamics of the immigrant experience. More and more people decide to leave their homes permanently and live as immigrants, even though migration often involves downward social mobility and financial insecurity. In addition to changes in their economic and legal status, immigrants also face the challenge of social and psychological adjustment in a foreign cultural environment.

\section{Identity Development in Acculturation}

In their theoretical paper on acculturation and identity, S. J. Schwartz et al. (2006) have argued that a coherent personal identity can function as an anchor to buffer the harmful effects of acculturative stress and guide the reconstruction of the social and cultural identity aspects of people in cultural transition. In recent years considerable attention in cross-cultural psychology has been focussed on the interplay between identity and cross-cultural adaptation in immigrants. Cross-cultural investigations undertaken with immigrant youth (Berry et al., 2006) and adults (Chen et al., 2008), and a meta-analytical review of more than 150 articles (Nguyen \& Benet-Martinez, 2013), have demonstrated that individuals who can successfully integrate aspects of the receiving culture with their ethnic culture of origin report greater psychological adaptation. Consistently, studies have suggested that conflict over the ethnocultural aspects of identity is linked to poorer psychological adaptation and increments in negative psychological symptoms in immigrants (Ward et al., 2011).

However, little research in acculturation deals with the question of how people in transition reconcile their identities as immigrants. We believe that one reason identity processes have received less consideration in acculturation research is the lack of reliable and valid assessment tools, specifically tailored for the immigrant population, to measure identity strategies in response to an acculturation identity crisis.

Although scales have been developed to study the identity structure of immigrants and people with multiple cultural affiliations, these measures focus on identity structures and outcomes (i.e., the perception and evaluation of a coherent identity) instead of capturing the processes underlying the development of an integrated and harmonious ethno-cultural identity. For example, the Bicultural Identity Integration Scale (Benet-Martinez \& Haritatos, 2005), a widely used measure of identity integration in multicultural settings, incorporates 
two components: cultural harmony (vs. conflict) and cultural blendedness (vs. distance). The former scale assesses the general evaluation of the identity, such as feeling balanced vs. being torn between two cultures, whereas the latter component captures the perception of the connections between the two identity aspects, i.e., blended vs. separated. However, this scale does not provide information about how bicultural individuals develop a harmonious identity, or what processes contribute to identity compartmentalization.

Similarly, the Ethno-cultural Identity Conflict Scale (Ward et al., 2011), another survey instrument developed for the acculturating population, measures whether immigrants experience a conflict over their ethnic and cultural identity aspects. However, it does not investigate the identity mechanisms underlying the development of a conflicted identity, or the ways that immigrants and ethnic minorities overcome an acculturation identity crisis. Although both scales have made essential contributions to the understanding of the development of identity structures in a multicultural setting, neither was designed to capture the underlying mechanisms.

A growing body of research points to the central role of an integrated and harmonious ethno-cultural and personal identity in the psychological adjustment of acculturating individuals (Berry et al., 2006; Chen et al., 2008; Nguyen \& Benet-Martinez, 2013; Ward et al., 2011). However, to develop a deeper insight into the dynamics of immigrant identity development, we need to shift our focus from structural differences in identity to the mechanisms underlying identity reconstruction during cultural transition. This approach would allow the identification of specific socio-cognitive strategies that help immigrants maintain a coherent identity, a clear sense of self, and high levels of self-esteem despite the difficulties and challenges in their daily lives.

\section{Social-cognitive Theory of Identity Styles}

In developmental psychology, social-cognitive strategies and identity processes have been intensively researched in relation to normative, age-related identity crises. In his socialcognitive theory on identity construction, Berzonsky $(1989,2011)$ has argued that stable individual differences can be identified in the way people respond to identity stress and process identity-relevant information. He proposed three distinct information processing and decision making strategies (i.e., identity styles) that underpin identity development; the informational, normative, and diffuse-avoidant styles.

Those who show a preference for the informational style actively engage in selfexploration and seek information and self-relevant feedback. They generally feel challenged by differences between their own views and the views of significant others or reference 
groups. Individuals with a normative strategy respond to identity stress with conformity and tend to rely on guidance from significant others or referential institutions when navigating changing environments. They experience high levels of discomfort (in the forms of anxiety and frustration) when their values and norms do not match the values and norms represented by significant others and/or the society. In order to reduce stress, they automatically internalize and adopt the values, rules and behaviours that are perceived to be socially desirable and appropriate. Finally, a diffuse-avoidant orientation involves strategic avoidance of self-relevant feedback and confrontation. Those who process social information on a diffuse-avoidant basis are motivated by situational demands and social factors, such as popularity and reputation. Consequently, they are present-oriented individuals who like to procrastinate and tend to make short-term decisions (Berzonsky, 2011).

Berzonsky (2008) has argued that every person has the cognitive capacity to apply all three social-cognitive styles; however, people usually have a dominant orientation, a strategy they consistently apply in response to identity stress throughout the lifespan and across situations. The social-cognitive perspective on identity development provides a potentially useful framework to investigate normative acculturation identity crises and to advance our understanding of the processes involved in creating a coherent immigrant identity.

\section{Overview of the Research}

The purpose of the studies reported here was to develop a survey instrument to measure social-cognitive strategies and identity orientations that underlie the identity development of acculturating individuals dealing with identity crises during cultural transition. Our research is situated within the theoretical framework of identity styles and builds on previous work by Berzonsky $(1989,2011)$.

The Identity Style Inventory (ISI, Berzonsky, 1989) was originally developed to assess the three social-cognitive identity styles previously described, but included a separate scale measuring identity commitment. This measurement tool has gone through a number of revisions and rigorous validation over the last 20 years (Berzonsky, 1992; Berzonsky et al., 2013; Smits, 2009) and has been used in numerous countries with different age groups mainly with the members of the cultural mainstream, although a few studies have also been undertaken with minority groups (S. J. Schwartz et al., 2005; S. J. Schwartz \& Montgomery, 2002) to investigate the processes underlying normative, age-related identity crises. However, it has never been used with culturally diverse participants facing an identity crisis evoked by cross-cultural transition. Therefore, we aimed at developing a version of the ISI specifically designed for the acculturating population that retains the main structure of the original 
measure. However, we also wanted to capture the specificity of cultural transition with the capacity to incorporate social-cognitive orientations, in addition to the three general styles, that might constitute effective strategies in an identity crisis induced by cultural transition.

The analysis of the psychometric properties of the scale was conducted with three samples to test the extent to which the Identity Style Inventory - Cultural Transition Version (ISI-CT) is a valid measure of identity styles with samples of ethnically and culturally diverse individuals confronting acculturative demands. The first study describes the scale construction process and tests the construct, convergent and discriminant validity of the new measure. Using confirmatory factor analysis, the second study further explores the construct validity of the scale. Finally, a longitudinal study is reported that examines the relationships among identity styles over time.

\section{Study 1: Scale Construction and Validation}

The aim of this study was to develop a version of the Identity Style Inventory to measure identity processing strategies during cultural transition and investigate its construct, convergent and discriminant validity by using measures of identity commitment, socialcognitive information processing, and social desirability.

\section{Identity Styles and Identity Commitment}

At times of identity crises, commitments are often questioned and re-evaluated through identity exploration (Marcia, 1966); research, however, suggests that identity reconstruction and new commitments can be achieved by various means, strategies, and behaviours (Berzonsky, 1989). Identity styles have been generally investigated in conjunction with identity commitment. Studies have consistently shown that those who score high on the diffuse-avoidant orientation report lower levels of commitment. In contrast, identity commitment has been positively related to the normative and informational orientations (see the meta-analysis by Bosch \& Card, 2012).

With respect to immigrant identity development, it is fair to assume that the normative style might reveal the opposite relationship with commitment to the immigrant identity. The two core elements of the normative style are (1) the automatic internalization of norms and values from significant others/society and (2) increased levels of anxiety and frustration caused by a perceived discrepancy between the norms/values of the individual and that of the society/significant others (Berzonsky, 2011). In a cross-cultural context, however, normative orientation can play out in two different ways. One can follow the rules by sticking to the value system of the home culture. On the other hand, a highly normative individual may automatically adopt the value and norm system of the host culture, given that going against 
the rules of the receiving society can induce high levels of anxiety and frustration.

Consequently, we tested two types of normative orientations: normative style to the country of origin (NCO) and normative style to the host society (NHS).

The relationship between identity styles and identity commitment was examined and we predicted the following associations:

H1. NCO was expected to be negatively related to identity commitment.

H2. NHS was predicted to positively correlate with identity commitment.

H3. The diffuse-avoidant style was predicted to negatively correlate with identity commitment.

H4. The informational style was hypothesized to be positively associated with identity commitment.

\section{Social-cognitive Criterion Measures}

Identity styles have revealed distinctive patterns of associations with a number of social-cognitive constructs, such as decision making strategies and cognitive reasoning, in the general population. For example, the informational style has been related to a strong need for rational explanations and reason, and high levels of experiential decision making strategies (Berzonsky, 2008; Berzonsky et al., 2013; Berzonsky \& Sullivan, 1992; Smits, 2009). Individuals with an informational orientation are characterized by open-mindedness and willingness to explore and consider alternatives. Consequently, this style has been linked to lower levels of cognitive closure (Crocetti et al., 2009; Soenens et al., 2005; Watson \& Morris, 2005). In contrast, people who prefer the diffuse-avoidant orientation make shortterm decisions depending on the demands of the situation and focus on immediate rewards; therefore, they tend to score lower on rational reasoning (Berzonsky, 2008; Berzonsky et al., 2013; Berzonsky \& Sullivan, 1992; Smits, 2009). The normative strategy has been associated with experiential, automatic, and heuristic information processing. More specifically, people who score high on the normative orientation heavily rely on their intentions when making important decisions and use significantly less rational decision making strategies (Berzonsky, 2008; Berzonsky et al., 2013; Smits, 2009). In addition, studies converge to link the normative style to higher levels of cognitive closure, need for predictability, and certainty (Crocetti et al., 2009; Soenens et al., 2005; Watson \& Morris, 2005).

To ensure that the Identity Style Inventory - Cultural Transition Version (ISI-CT) is also a valid measure of identity processes for people in cultural transition, the extent to which the subscales of the ISI-CT were related to measures of information processing was examined. Three instruments were chosen to assess the convergent validity of the ISI-CT: the 
Need for Cognition Scale measuring rational decision making, the Faith in Intuition Scale assessing experiential decision making strategies, and the Need for Cognitive Closure Scale capturing automatic processing. The relationship between identity styles and social desirability was also examined to assess discriminant validity (D. T. Campbell \& Fiske, 1959; King \& Bruner, 2000). We expected that measures not affected by response bias should reveal null correlations with scales measuring social desirability, demonstrating that the central measures being tested do not merely assess the simple tendency to agree to statements that convey socially favourable impressions.

Based on empirical studies discussed above, we proposed the following hypotheses for the relationships between the ISI-CT subscales and the social-cognitive criterion measures:

H5. The informational style would have a positive association with rational reasoning and experiential decision making, and a negative correlation with need for closure.

H6. The normative styles would show a negative relationship with rational decision making and a positive correlation with experiential reasoning and need for closure.

H7. The diffuse-avoidant style was expected to yield a negative relationship with rational decision making and to be unrelated to experiential reasoning.

H8. No or weak positive correlations were expected between the ISI-CT subscales and social desirability.

\section{Method}

\section{Construction of the Identity Style Inventory - Cultural Transition Version}

In the first step, a 56-item pool was created by combining items from previous versions of the ISI (e.g., the ISI-3 and ISI-4 by Berzonsky, 1992b; Smits, 2009) ${ }^{1}$. Second, items were rephrased to reflect the context of cultural transition (e.g., original item: 'I've spent a great deal of time thinking seriously about what I should do with my life', modified item: 'I've spent a great deal of time thinking seriously about what I should do with my life in this country'). Furthermore, statements referring to religious beliefs and political views were modified to beliefs about multiculturalism and views about immigration (e.g., original item: "I've spent a lot of time reading and trying to make some sense out of political issues.", modified item: "I've spent a lot of time reading and trying to make some sense out of issues that arise when people from many cultures live together in one society."). This step was necessary to ensure that we measured identity styles in the context of acculturation.

Finally, the normative scale was revised and further divided into two subscales. One set of items captured a normative orientation to the country of origin, while another set of 
statements indicated normative information processing in favour of the host society (e.g., country of origin: "I've more-or-less always operated according to the values and norms with which I was brought up.", host society: “I've more-or-less always tried to operate according to the values and norms which are represented by the locals.").

\section{Procedure and Participants}

The study was conducted with immigrants residing in New Zealand, one of the most diverse countries in the OECD, where one in four people was born overseas (Statistics New Zealand, 2014). To participate in this study, subjects had to meet the following sampling criteria: participants must have 1) been born outside of New Zealand, 2) come to New Zealand when they were at least 16 years old, 3) been living in New Zealand at the time of data collection, 4) been older than 18 years of age, and 5) been non-refugee immigrants. Participants were invited to complete an online survey through social media and registered ethnic and cultural organizations in New Zealand. Respondents did not receive any compensation for their participation.

The final sample included 180 non-refugee immigrants residing in New Zealand. Of participants, 65\% were female $(n=117)$ and $70 \%$ were non-native speakers of English $(n=$ 126) with an average self-reported English language proficiency of $M=6.10$ (on a 7-point scale; $S D=.96)$. Respondents ranged in age from 18 to $78(M=39$ years, $S D=12.73$ years $)$. The average time spent in the country was $M=9.49$ years $(S D=11.11$ years). The sample included international students $(n=25)$, employment pass holders $(n=17)$, permanent residents $(n=86)$, New Zealand/dual citizens $(n=51)$, and one Australian citizen. Even though the majority of participants had gained permanent residency or New Zealand citizenship, they identified with the immigrant category to a great extent (on a 7-point scale, $M=4.86, S D=1.94)$. Participants were characterized by a diverse cultural background, originating from Eastern Europe $(n=58)$, Western Europe $(n=34)$, North America $(n=35)$, East-, South-, Southeast Asia and the Pacific $(n=28)$, Africa $(n=15)$, South America $(n=$ $6)$, and the Middle East $(n=4)$.

\section{Materials}

The survey included questions pertaining to demographic information, the ISI-CT, the Rational-Experiential Inventory, the Need for Closure Scale, and the Marlowe-Crowne Social Desirability Scale.

Identity Style Inventory - Cultural Transition Version (ISI-CT). The final item pool consisted of 45 items assessing four types of social-cognitive identity strategies (i.e., informational, diffuse-avoidant, normative style to the host society, and normative style to the 
country of origin), and 11 items measuring commitment to the receiving society, the decision to immigrate, and the immigrant identity. The commitment scale consisted of both positively and negatively phrased items, whereas identity styles were measured by positively phrased items, exclusively. The instructions prefacing the items included a prompt: You will find a number of statements about values, attitudes, and ways of dealing with issues as an immigrant in a foreign country. Next, participants were asked to think about the time that had passed since they arrived to New Zealand and to judge on a 5-point Likert scale (anchored at $1=$ not like me at all, $5=$ very much like me) the extent to which a statement described them and their behaviour as immigrants in New Zealand.

Rational-Experiential Inventory (REI). The REI was designed to measure individual differences in thinking styles. In this study a 10-item version of the instrument was used. Five of the items are selected statements from the Need for Cognition Scale (Cacioppo $\&$ Petty, 1982) that represent rational information processing. The remaining five items belong to the Faith in Intuition Scale developed by Epstein, Pacini, Denes-Raj, and Heier (1996) to capture experiential-intuitive information processing. The Need for Cognition scale contains both positively and negatively phrased items, and higher scores indicate preferences toward rational-analytical information processing. The Faith in Intuition scale is formed by positively phrased statements with higher scores indicating the primary use of experiential reasoning. Participants used a 5-point Likert scale (anchors of $1=$ completely false, $5=$ completely true) in response to statements such as "I prefer complex to simple problems" (Need for Cognition) or "I trust my initial feelings about people" (Faith in Intuition). The construct and convergent validity of the measures has been previously confirmed (Epstein et al., 1996). In the present study, both scales showed good reliability (Need for Cognition: $\alpha=$ .73 , Faith in Intuition: $\alpha=.85$ ).

Need for Cognitive Closure (NFC). A brief version of the Need for Closure Scale was employed in order to measure the cognitive-motivational basis of decision making (Roets \& Van Hiel, 2011). The scale comprises 15 items and covers five main domains: need for order (e.g., "I enjoy having a clear and structured mode of life."), need for predictability (e.g., "I dislike unpredictable situations."), decisiveness (e.g., "When I have made a decision, I feel relieved."), intolerance of ambiguity (e.g., "I don't like situations that are uncertain."), and closed-mindedness (e.g., "I dislike questions which could be answered in many different ways."). Statements were rated on a 6-point scale ranging from $1=$ strongly disagree to $6=$ strongly agree. The abridged version shows a strong relationship $(r=.95)$ with the original 41-item scale and has proved to be a valid and reliable alternative to measure individual 
differences in the need for cognitive closure (Roets \& Van Hiel, 2011). In the present investigation, the scale demonstrated acceptable internal consistency $(\alpha=.87)$.

Social Desirability. The 13-item version of the Marlowe-Crowne Social Desirability Scale (MCSDS) designed by Reynolds (1982) was included to examine tendencies for socially desirable responses. Participants assessed items such as "I'm always willing to admit when I make a mistake." on a true-false basis. The total score of the scale ranged from 0 to $13(M=7.24, S D=2.86)$ with higher scores indicating a stronger inclination for social desirability. The reliability and validity of this shortened version of the scale have been previously supported by Reynolds (1982), and the measure yielded acceptable internal consistency in the present study as well (Kuder-Richardson $20=.69$ ).

\section{Statistical Analysis}

Structural validity. Parallel analysis (PA) and exploratory factor analysis (EFA) were employed to explore the structure underlying the identity style items and identify the number of factors to be retained. The PA with Monte Carlo simulations was conducted using raw data permutation and 100 randomly generated data sets. For the EFA, maximum likelihood estimation and orthogonal (varimax) rotation were applied. In addition, a principal component analysis was applied to investigate the single factor structure of the immigrant commitment scale.

Internal consistency. Three indices were used to examine the internal consistency of the ISI-CT subscales: Cronbach's alpha, average inter-item correlation, and item-total correlation. Although the Cronbach's alpha coefficient is the most widely used indicator of reliability (Shrout \& Lane, 2012), with values higher than the .70 threshold indicating acceptable internal consistency (Kaplan \& Saccuzzo, 1982), it is also a function of the number of items a scale contains (Nunnaly, 1978). As an alternative to the Cronbach's alpha, average inter-item correlations were calculated. This method is not sensitive to the number of items a scale comprises and is a clear and straightforward indicator of internal consistency (Clark \& Watson, 1995). Following recommendations by Briggs and Cheek (1986), average inter-item correlations should fall in the range of .15-.50. Finally, to investigate the reliability of specific items, corrected item-total correlations were analysed. As a minimum, a weak correlation $(r \geq .20)$ was expected between each item and the corrected total scale.

Convergent and discriminant validity. Bivariate correlations were calculated to examine the convergent and discriminant validity of the ISI-CT. As previous research has shown that the preferences for formal versus intuitive reasoning vary across cultures (Norenzayan, Smith, Kim, \& Nisbett, 2002), we controlled for cultural background (Western 
vs. non-Western) and language proficiency in our analyses. The relationship between the ISICT subscales and demographic variables was also examined using bivariate correlations and independent samples t-tests.

\section{Results}

\section{Parallel Analysis and Exploratory Factor Analysis: Identity Styles for Cultural Transition}

Parallel analysis indicated a maximum of six factors to be retained with the first five components having an eigenvalue higher than two. In the second step, an EFA was conducted. Eigenvalues and scree plot were examined to identify the potential number of dimensions underlying our data set. Both criteria indicated five factors to be retained. Items with a communality value lower than 0.2 were dropped. The final five-factor structure explained $43.14 \%$ of total variance and revealed good adequacy $(\mathrm{KMO}=.74)$ as well as fit $\left(\chi^{2}(131)=151.74, n s\right.$. $)$ with factor loadings higher than .42 and cross-loadings lower than .32. The second, third and fourth factors included items capturing diffuse-avoidance (DA; 5 items), normative orientation toward the country of origin (NCO; 5 items), and normative orientation toward the host society (NHS; 4 items), respectively. The first and fifth factors consisted of items originally related to the informational style. The first factor comprised four items referring to consideration of alternatives and critical information assessment, i.e., analytical informational strategies (AI). In contrast, the fifth dimension captured behaviours and strategies related to information seeking through interpersonal contact, i.e., exploratory informational strategies, with four items (EI, Table 2.1.).

All five scales demonstrated acceptable internal consistency. AI had a reliability of $\alpha$ $=.79$. The corrected item-total correlations ranged between $r=.49$ and $r=.67$, and the average inter-item correlation was $r=.49$. EI had a Cronbach's alpha of $\alpha=.67$ with corrected item-total correlations ranging from $r=.36$ to $r=.53$ and an average inter-item correlation of $r=.34$. The Cronbach's alpha for DA was $\alpha=.71$, and the corrected item-total correlations ranged from $r=.37$ to $r=.55$ with an average inter-item correlation of $r=.33$. NCO also demonstrated good reliability (Cronbach's alpha: $\alpha=.74$ ) with acceptable corrected item-total correlations (from $r=.41$ to $r=.62$ ) and average inter-item correlation ( $r$ $=.36$ ). The reliability for the NHS scale was $\alpha=.73$, and the corrected item-total correlations varied between $r=.44$ and $r=.60$ with an average inter-item correlation of $r=.41$. 
Table 2.1. Identity Style Inventory - Cultural Transition Version: Factor loadings and descriptive statistics (Study 1).

\begin{tabular}{|c|c|c|c|c|c|c|c|}
\hline & $\mathrm{NCO}(9.5 \%)$ & $\mathrm{AI}(9.4 \%)$ & DA $(8.9 \%)$ & NHS $(8.1 \%)$ & EI $(7.2 \%)$ & $M$ & $S D$ \\
\hline I automatically adopt and follow the values I was brought up with. & .77 & & & & & 2.92 & 1.17 \\
\hline I've more-or-less always operated according to the values and norms with which I was brought up. & .70 & & & & & 3.16 & 1.15 \\
\hline I strive to achieve the goals that my family and friends from my home country hold for me. & .56 & & & & & 2.16 & 1.22 \\
\hline I prefer to deal with situations according to the social norms and standards of my home country. & .52 & & & & & 2.69 & 1.07 \\
\hline I never question what I want to do with my life because I tend to follow what my family expects me to do. & .42 & & & & & 1.57 & .84 \\
\hline When making important decisions, I like to think about my options. & & .78 & & & & 4.52 & .70 \\
\hline When facing a decision, I try to analyse the situation in order to understand it. & & .78 & & & & 4.35 & .77 \\
\hline When I have to make a decision, I like to spend a lot of time thinking about my options. & & .60 & & & & 4.13 & .85 \\
\hline When facing a decision, I take into account different points of view before making a choice. & & .58 & & & & 3.89 & .93 \\
\hline I try not to think about or deal with problems as long as I can. & & & .68 & & & 2.09 & 1.14 \\
\hline When I have to make a decision, I try to wait as long as possible in order to see what will happen. & & & .57 & & & 2.43 & 1.17 \\
\hline I try to avoid personal situations that will require me to think a lot and deal with them on my own. & & & .57 & & & 2.93 & 1.15 \\
\hline When I know a situation is going to cause me stress, I try to avoid it. & & & .55 & & & 1.99 & 1.16 \\
\hline Sometimes I refuse to believe a problem will happen, and things manage to work themselves out. & & & .44 & & & 2.43 & 1.16 \\
\hline I've more-or-less always tried to operate according to the local values and norms. & & & & .73 & & 3.31 & .90 \\
\hline I automatically adopt and follow the values of the society in which I live. & & & & .62 & & 3.12 & 1.00 \\
\hline I think it's better to adopt the value system of the host country, if it helps me to avoid conflicting situations. & & & & .61 & & 2.82 & 1.11 \\
\hline I prefer to deal with situations according to the social norms and standards of the country in which I live. & & & & .55 & & 3.56 & 1.00 \\
\hline $\begin{array}{l}\text { I've spent a lot of time reading and trying to make some sense out of issues that arise when people from many cultures } \\
\text { live together in one society. }\end{array}$ & & & & & .69 & 3.04 & 1.34 \\
\hline $\begin{array}{l}\text { I've spent a good deal of time reading and talking to others about the values and rules of the country in which I } \\
\text { currently live. }\end{array}$ & & & & & .64 & 3.24 & 1.18 \\
\hline I've spent a great deal of time thinking seriously about what I should do with my life in this country. & & & & & .51 & 3.21 & 1.28 \\
\hline I've spent a lot of time and talked to a lot of people trying to develop a set of values that makes sense to me. & & & & & .50 & 3.42 & 1.29 \\
\hline
\end{tabular}

Note. Cross-loadings smaller than .40 are supressed. AI, Analytical Informational Style, EI, Exploratory Informational Style, DA, Diffuse-avoidant Style, NCO, Normative Style toward the Country of Origin, NHS, Normative Style toward the Host Society. 


\section{Principal Component Analysis: Identity Commitment}

Principal Component Analysis was applied to examine the single factor structure of the modified commitment scale. The eigenvalues and the scree plot yielded one possible dimension underlying the data, thus supporting the single factor model of the scale. Items with communalities lower than 0.2 were eliminated, and the final analysis revealed six items loading onto the same latent component, which explained $46 \%$ of total variance. The scale demonstrated acceptable reliability $(\alpha=.76)$ and average inter-item correlation $(r=.34)$. Factor loadings, means, standard deviations, and item-total correlations are reported in Table 2.2 .

Table 2.2. Identity Commitment Scale: factor loadings, item-total correlations, and descriptive statistics (Study1).

\begin{tabular}{|c|c|c|c|c|}
\hline & $\begin{array}{l}\text { EFA Factor } \\
\text { Loading }\end{array}$ & $\begin{array}{l}\text { Item-total } \\
\text { Correlation }\end{array}$ & $M$ & $S D$ \\
\hline I know what I want to do with my future in this country. & .84 & .69 & 3.58 & 1.32 \\
\hline I'm not sure what I want to do in the future in this country. (Reversed) & .82 & .67 & 3.63 & 1.45 \\
\hline $\begin{array}{l}\text { I'm not sure whether I should stay here (or move to another country). } \\
\text { (Reversed) }\end{array}$ & .72 & .54 & 3.53 & 1.51 \\
\hline I am not sure what I want out of life. (Reversed) & .57 & .40 & 4.06 & 1.24 \\
\hline I really like this country; it was the right decision to move here. & .56 & .39 & 4.11 & 1.07 \\
\hline I'm not sure any more which values I really hold. (Reversed) & .50 & .35 & 4.36 & .97 \\
\hline
\end{tabular}

\section{Convergent and Discriminant Validity}

Bivariate correlations between the ISI-CT subscales and the criterion measures were calculated to investigate the convergent and discriminant validity of the instrument (Table 2.3.). First, correlations among identity styles were examined. The two informational factors (i.e., analytical and exploratory) yielded a positive and weak correlation $(r=.25, p=.001)$. Diffuse-avoidance was positively and weakly related to both NCO $(r=.25, p<.001)$ and NHS ( $r=.22, p=.004)$. Furthermore, it was negatively and weakly associated with the analytical informational style $(r=-.18, p=.013)$, but showed no relationship with the exploratory informational style. In addition, the two normative subscales yielded a positive but weak correlation $(r=.23, p=.002)$. Contrary to our hypothesis, both informational scales were unrelated to immigrant commitment. DA $(r=-.37, p<.001)$ and NCO $(r=-.24$, $p=.001)$ were negatively related to identity commitment, whereas NHS showed a positive association with immigrant commitment $(r=.25, p=.001)$, all in line with our predictions. 
Next, the associations between identity styles and cognitive measures were scrutinized. Both informational measures positively and moderately correlated with rational decision making (analytical: $r=.35, p<.001$ and exploratory: $r=.35, p<.001$ ). Diffuseavoidance strongly and negatively correlated with rational decision making $(r=-.45, p<$ $.001)$ and yielded a positive, weak to moderate relationship with need for closure $(r=.29, p<$ $.001)$. NCO was negatively and weakly related to rational decision making $(r=-.20, p=$ $.013)$ and positively and moderately linked to need for closure $(r=.35, p<.001)$. NHS showed a negative and weak, albeit marginally significant, association with rational reasoning $(r=-.15, p=.064)$, and a positive, weak relationship with intuitive decision making $(r=.16, p=.043)$.

Discriminant validity was tested by examining the relationship between social desirability and identity styles. NHS $(r=.16, p=.044)$ showed a positive but weak association with social desirability, whereas diffuse-avoidance yielded a negative correlation $(r=-.21, p=.008)$ with a weak effect size.

\section{Additional Analyses}

Correlations were examined between the identity styles and demographic variables, including age, length of stay, and English language proficiency. None of the identity styles were significantly related to age or language proficiency. However, the analytical informational style was negatively correlated with length of stay $(r=-.17, p=.023)$, whereas NHS showed a positive, weak association $(r=.16, p=.029)$. Group differences were calculated based on gender and cultural background. There was no difference between males and females in the use of identity styles during cultural transition. When comparing Western and non-Western participants in New Zealand, significant differences were found in DA and NCO; $t(178)=-2.79, p=.006$, Cohen's $d=.42$ and $t(178)=-2.53, p=.012$, Cohen's $d=$ .38 , respectively. More specifically, non-Western respondents scored significantly higher on DA $(M=2.63, S D=.83)$ and $\mathrm{NCO}(M=2.72, S D=.81)$ compared to their Western peers (DA: $M=2.27, S D=.75 ; \mathrm{NCO}: M=2.41, S D=.73$ ). 
Table 2.3. Descriptive statistics and correlations among identity styles, criterion measures, and demographic variables (Study1).

\begin{tabular}{|c|c|c|c|c|c|c|c|c|c|c|c|c|c|c|}
\hline & 1 & 2 & 3 & 4 & 5 & 6 & 7 & 8 & 9 & 10 & 11 & 12 & $M$ & $S D$ \\
\hline \multicolumn{15}{|l|}{ Identity Styles } \\
\hline 1. Analytical Informational & 1 & & & & & & & & & & & & 4.22 & .64 \\
\hline 2. Exploratory Informational & $.25^{* *}$ & 1 & & & & & & & & & & & 3.23 & .90 \\
\hline 3. Diffuse-avoidant & $-.18^{*}$ & .03 & 1 & & & & & & & & & & 2.37 & .79 \\
\hline 4. Normative to the Country of Origin & -.08 & -.02 & $.25^{* *}$ & 1 & & & & & & & & & 2.50 & .77 \\
\hline 5. Normative to the Host Society & .03 & .04 & $.22^{* *}$ & $.23^{* *}$ & 1 & & & & & & & & 3.20 & .75 \\
\hline \multicolumn{15}{|l|}{ Convergent Validity } \\
\hline 6. Identity Commitment & .07 & -.02 & $-.37^{* *}$ & $-.24^{* *}$ & $.25^{* *}$ & 1 & & & & & & & 3.88 & .86 \\
\hline 7. Need for Closure & .09 & .06 & $.29^{* *}$ & $.35^{* *}$ & .12 & $-.18^{*}$ & 1 & & & & & & 3.47 & .86 \\
\hline 8. Rational Decision Making & $.35^{* *}$ & $.35^{* *}$ & $-.45^{* *}$ & $-.20^{* *}$ & $-.15^{+}$ & $.14^{+}$ & $-.26^{* *}$ & 1 & & & & & 3.99 & .70 \\
\hline 9. Intuitive Decision Making & .06 & .01 & .06 & .08 & $.16^{*}$ & $.16^{*}$ & .09 & .02 & 1 & & & & 3.63 & .74 \\
\hline \multicolumn{15}{|l|}{ Discriminant Validity } \\
\hline 10. Social Desirability & .08 & $-.13^{+}$ & $-.21^{* *}$ & -.06 & $.16^{*}$ & $.23^{* *}$ & $-.26^{* *}$ & .00 & -.01 & 1 & & & 7.24 & 2.86 \\
\hline \multicolumn{15}{|l|}{ Demographic Variables } \\
\hline 11. Length of Stay & $-.17^{*}$ & -.07 & .01 & -.10 & $.16^{*}$ & $.24^{* *}$ & .03 & -.11 & .07 & .11 & 1 & & 9.49 & 11.12 \\
\hline 12. Age & -.11 & .02 & .01 & -.10 & .09 & $.22^{* *}$ & .02 & -.03 & .08 & .10 & $.77^{* *}$ & 1 & 39.04 & 12.73 \\
\hline
\end{tabular}

Note. ${ }^{* *} \mathrm{p}<.01 ; * \mathrm{p}<.05 ;{ }^{+} \mathrm{p}<.01$. The correlations among the identity styles and the criterion measures controlling for cultural background and English language proficiency. Predicted correlations are boldfaced. 


\section{Discussion}

The study examined the factor structure, along with the convergent and discriminant validity of the ISI-CT.

\section{Structure of the Identity Style Inventory - Cultural Transition Version}

Initial analyses with a sample of immigrants residing in New Zealand produced five independent identity styles operating during cultural transition. In line with our hypotheses, two types of normative style were identified demonstrating that normative orientation to the

host society (NHS) and normative orientation to the country of origin (NCO) are not opposite endpoints of the same dimension, but relatively independent social-cognitive orientations for acculturating individuals. In addition, two forms of the informational style emerged: analytical informational (AI) and exploratory informational (EI). The former captures inward directed information processing strategies, such as the consideration of alternatives in decision making, whereas the latter refers to the exploration of alternatives mainly through interpersonal contact, such as talking to others. Furthermore and as expected, the EFA produced a distinct factor measuring the diffuse-avoidant (DA) style. All scales demonstrated acceptable internal consistency and showed weak to moderate correlations with each other.

\section{Convergent and Discriminant Validity}

To further investigate the construct validity of the ISI-CT, convergent and discriminant validity were established by using measures of identity commitment, cognitive processing, and social desirability. As expected, and in line with previous studies, the two informational styles positively and moderately correlated with rational, effortful and logical decision making strategies. Contrary to our hypotheses, however, both styles were unrelated to intuitive decision making and need for closure. In addition, and unexpectedly, the two strategies showed no significant relationship with immigrant commitment, contrary to prior research (Bosch \& Card, 2012). In general, the analytical and exploratory informational styles related to criterion measures in similar ways, which indicates that the two styles have similar cognitive-motivational bases.

As predicted, the diffuse-avoidant style was negatively correlated with rational reasoning. It also showed a positive, moderately strong positive relationship with need for closure, which was not hypothesized. Although, previous studies have found no associations between the composite measure of need for closure and the diffuse-avoidant strategy, there is evidence showing that the style is linked to lower levels of openness (Berzonsky \& Sullivan, 1992; Duriez \& Soenens, 2006; Duriez, Soenens, \& Beyers, 2004) and tolerance to new ideas (Berzonsky \& Kuk, 2005), which are both key aspects of need for closure. In summary, these 
results suggest that immigrants who process on a predominantly diffuse-avoidant basis use less rational and logical reasoning and tend to rely on automatic processing in decision making. Furthermore, the diffuse-avoidant style was negatively linked to immigrant commitment indicating lower levels of willingness to commit to the host society and the immigrant identity.

The theoretical distinction between the two normative styles is further supported by the finding that they are associated with various criterion measures in different ways. Our results suggest that NCO and NHS manifest different mechanisms underlying problem solving behaviour and cognitive reasoning in cultural transition. Higher NCO was related to increased need for closure, but lower levels of rational processing. In contrast, NHS was unrelated to need for closure and positively associated with experiential decision making. These results are not surprising, as being normative towards the country of origin demands less effortful and more automatic processing. On the other hand, the adoption of the valueand norm system of a new culture involves a great amount of uncertainty and requires reasonable levels of open-mindedness and intentionality to identify the socially desirable behaviours and attitudes in the receiving country.

Findings showed that three out of the five identity styles were unrelated to social desirability. NHS and the diffuse-avoidant style revealed significant but weak correlations with the measure. Considering that none of the styles showed a substantial relationship with social desirability, the discriminant validity of the scales was supported suggesting that the ISI-CT measures more than just a tendency to show agreement or disagreement.

Additional analyses indicated no gender difference in the use of identity styles. However, non-Western respondents used diffuse-avoidant and NCO strategies to a greater extent compared to their Western peers. This finding highlights the importance of cultural distance and other potential socio-cultural factors affecting the differential use of identity styles in a cross-cultural context.

\section{Study 2: Confirming the Factor Structure of the ISI-CT}

The objective of Study 2 was to test the five-factor structure of the identity styles during cultural transition and the single factor structure of the identity commitment scale by using confirmatory factor analysis. In addition, we examined the correlations among identity styles and identity commitment to replicate the findings from study 1 , and we further investigated group differences based on gender, previous overseas experience, language proficiency, and ethnic visibility. 


\section{Method}

\section{Procedure and Participants}

Participants were invited to complete an online, anonymous survey on immigrant identity through social media and ethno-cultural organizations. To participate in the study, respondents must have been 18 years of age or older, been living in a country different to their home countries, and migrated to the receiving country as adults. At the end of the survey, participants were offered to enter a lucky draw for Amazon vouchers; however, contact information was collected in a way that did not allow us to connect email addresses to survey responses.

The sample included 479 immigrants (53\% male) with a mean age of $M=29$ years $(S D=9$ years). Participants originated from North America (32\%), Western Europe (24.3\%), Eastern Europe (16.1\%), East-, South-, Southeast Asia and the Pacific (9.2\%), South America (8.6\%), Australia and New Zealand (5\%), Africa (2.5\%), and the Middle East (2.3\%). The majority of receiving countries were located in Western Europe (38.9\%) followed by East-, South-, Southeast Asia and the Pacific (19.9\%), North America (16.9\%), Australia and New Zealand (12.1\%), South America (4\%), Eastern Europe (3.8\%), Africa (2.9\%), and the Middle East (1.5\%). More than half of the sample (55.9\%) had previous overseas experience and $69.7 \%$ were non-native speakers of the official language of the country of residence. The average self-reported English language proficiency (the language of the survey) was $M=7.21$ (on an 8-point scale, $S D=.99$ ). The majority of participants were recent immigrants, who had been living in the host society for no longer than two years $(65 \%)$ at the time of data collection, and $40.5 \%$ considered themselves to be a visible minority in the host society. With respect to visa status, most participants had a working visa/employment pass $(33.5 \%)$, which was followed by people holding a student visa (27\%), resident visa (24.5\%), dual citizenship (7.7\%), and other types of visa (7.3\%).

\section{Materials}

The ISI-CT was administered as part of a larger survey that included questions pertaining to demographic variables and scales measuring identity constructs and crosscultural adaptation. In the present investigation, the variables of interest were the identity styles and the identity commitment subscale from the ISI-CT.

\section{Data Analysis}

Confirmatory factor analyses (CFA) were performed to test the construct validity of the ISI-CT. Four models were tested and compared. In Model 1, we employed a one-factor solution with all items loading onto a single latent factor. Model 2 tested a five-factor 
solution using item-level CFA. In Model 3, we tested the same five-factor solution but allowed error terms to covary. In Model 4, parcelling was applied to eliminate sampling error and reduce the chance of residuals being correlated (Little, Cunningham, Shahar, \& Widaman, 2002). Items were randomly assigned into one of two parcels for each scale. Parcelled models are more accurate at estimating the fit to the data when the sample size is relatively small, and they are more parsimonious as the latent constructs are defined by fewer parameters (Bagozzi \& Edwards, 1998). In a separate analysis, item-level CFA was applied to test the single factor structure of the identity commitment scale.

Three indices were used to assess the model fit: the comparative fit index (CFI), the root mean square error of approximation with confidence interval (RMSEA), and the standardized root mean square residual (SRMR). Following the recommendations by $\mathrm{Hu}$ and Bentler (1999), models with CFI higher than .90 indicated marginally acceptable fit, whereas models with CFI higher than .95, RMSEA lower than .06, and SRMR lower than .08 were considered to demonstrate good fit to the data. When comparing models, AIC, BIC, and ABIC difference tests were calculated. A difference of 10 in the AIC, BIC, and ABIC were considered to indicate improvement in the model fit (Raftery, 1995).

\section{Results and Discussion}

\section{Confirmatory Factor Analysis}

Results of the CFA and model comparisons are reported in Table 2.4. Model 1 (all items loading onto one latent factor), as expected, yielded a very poor fit to the data with a CFI well below .95 and RMSEA and SRMR well above .06 and .08, respectively. Model 2 (five-factor solution) showed a much improved fit and a substantial change in the AIC, BIC, and ABIC; however, the CFI was still below the recommended threshold of .95 and modification indices indicated a number of correlating error terms. Therefore, we allowed these error terms to covary and reran the item-level CFA with five factors (Model 3). In Model 3, fit indices revealed a marginally acceptable fit with a CFI higher than .90, RMSEA and SRMR lower than .06 and .08, respectively. Finally, in Model 4, a parcelled model was tested, which yielded an excellent fit to the data and a substantial change in the AIC, BIC, and ABIC compared to Model 3 (see Table 2.4). In Model 4, all parameters from the observed variables to the latent constructs were significant and the regression weights were high (average $=.74$, Figure 2.1.). No significant covariances were identified.

The initial item-level CFA for commitment yielded a marginally acceptable fit to the data and modification indices indicated two significant error covariances (see Table 2.4). By letting these residual errors correlate, fit indices substantially improved indicating a good fit 
to the data. All parameters were significant and the regression weights were high (average = .64, Figure 2.2.). All scales demonstrated acceptable internal consistency with corrected itemtotal correlations and average inter-item correlations ranging in the expected fashion (see Table 2.5.). Taken together, these analyses confirmed the five-factor structure of the ISI-CT and the unidimensional identity commitment scale.

\section{Correlational Analysis}

Bivariate correlations were examined among the identity styles and identity commitment (see Table 2.5.). The analytical informational style was positively and weakly associated with the exploratory informational style $(r=.30, p<.001)$ and immigrant commitment $(r=.15, p=$ $.001)$, and showed a weak, negative link to the diffuse-avoidant style $(r=-.24, p<.001)$ and NCO $(r=-.17, p<.001)$. Similarly, the exploratory informational style negatively and weakly correlated with diffuse-avoidance $(r=-.13, p=.006)$ and $\mathrm{NCO}(r=-.15, p=.001)$, but showed a positive, weak association with NHS $(r=.12, p=.007)$. The diffuse-avoidant style had a positive, weak correlation with $\mathrm{NCO}(r=.28, p<.001)$ and a negative, moderately strong relationship with immigrant commitment $(r=-.36, p<.001)$. NHS was positively and weakly related to immigrant commitment $(r=.16, p<.001)$ and unrelated to NCO. In contrast, NCO yielded a negative but weak correlation with commitment $(r=-.20, p$ $<.001$ ). These results confirmed the patterns of relationships found in study 1 . In addition, identity styles yielded only weak to moderate associations with one another and identity commitment, which provides further evidence for the discriminant validity of the measure.

\section{Group Comparisons}

There were no significant differences in the use of identity styles according to proficiency in the official language of the receiving country (native vs. non-native speakers), former overseas experience, and gender. A significant but small difference was found between visible and non-visible minorities in the use of EI, $t(477)=2.44, p<.05$, Cohen's $d$ $=.23$. Specifically, participants who considered themselves to be visible ethnic minorities in their host societies scored higher on the exploratory informational style $(M=3.77, S D=.70)$ compared to non-visible ethnic minorities $(M=3.60, S D=.75)$. It is, however, worth noting that one quarter of participants $(25 \%)$ were immigrants from Western countries (e.g., the United States or Europe) living in a non-Western context (e.g., Southeast Asia or the Middle East) and a lesser, but still significant, proportion of the participants (19\%) were immigrants from a non-Western context (e.g., Southeast Asia or Africa) residing in the United States, Europe or Australia. Therefore, visibility might be an indicator of cultural distance. When the dissimilarity between the heritage and the host culture is large, new immigrants might need to 
spend more time and effort into gaining enough information to understand the value discrepancies that can arise when people from greatly different cultures live together in the same environment.

In summary, study 2 confirmed the factor structure of the ISI-CT with a sample of immigrants from diverse cultural backgrounds. Furthermore, we replicated the patterns of correlations among the ISI-CT subscales and group differences in the use of identity styles. In addition, findings with respect to differences between visible and non-visible immigrants further highlighted the impact of socio-cultural variables (e.g., cultural distance) on identity processes in cultural transition. It is also worth emphasizing that the sample for study 2 was much more diverse in terms of destinations and origins than study 1, which further supports the generalizability of the findings and the utility of the measure across diverse groups of acculturating individuals. 
Table 2.4. Fit indices for each model and model comparisons (Study 2).

\begin{tabular}{|c|c|c|c|c|c|c|c|c|c|c|c|c|c|c|}
\hline Model & $\chi^{2}$ & $d f$ & $\chi^{2} / d f$ & RMSEA & $90 \% C I$ & CFI & SRMR & $A I C$ & $B I C$ & $A B I C$ & Comparison & $\triangle A I C$ & $\triangle B I C$ & $\triangle A B I C$ \\
\hline \multicolumn{15}{|l|}{ Identity Styles } \\
\hline Model 1 (single factor) & 1655.26 & 209 & 7.92 & .120 & $.115 ; .126$ & .316 & .116 & 29165.60 & 29440.93 & 29231.46 & - & - & - & - \\
\hline Model 2 (five factors, item-level) & 459.110 & 199 & 2.31 & .052 & $.046 ; .059$ & .877 & .063 & 27922.69 & 28239.74 & 27998.53 & Model 1 and 2 & 1242.91 & 1201.19 & 1232.93 \\
\hline $\begin{array}{l}\text { Model } 3 \text { (five factors, item-level with } \\
\text { covariances) }\end{array}$ & 333.330 & 190 & 1.75 & .040 & $.033 ; .047$ & .932 & .056 & 27800.17 & 28154.76 & 27884.98 & Model 2 and 3 & 122.52 & 84.98 & 113.55 \\
\hline Model 4 (five factors, parcelled) & 44.415 & 25 & 1.78 & .040 & $.020 ; .059$ & .978 & .032 & 10673.604 & 10840.47 & 10713.52 & Model 3 and 4 & 17126.566 & 17314.29 & 17171.46 \\
\hline \multicolumn{15}{|l|}{ Identity Commitment } \\
\hline Model 1 (single factor) & 52.370 & 9 & 5.82 & .100 & $.075 ; .127$ & .940 & .044 & 8277.27 & 8352.36 & 8295.33 & - & & & \\
\hline $\begin{array}{l}\text { Model } 2 \text { (single factor with } \\
\text { covariances) }\end{array}$ & 14.277 & 7 & 2.04 & .047 & $.006 ; .081$ & .990 & .026 & 8230.78 & 8314.22 & 8250.74 & Model 1 and 2 & 46.49 & 38.14 & 44.59 \\
\hline
\end{tabular}

Note. RMSEA, Root Mean Square Error of Approximation; 90\% CI, Confidence Interval for RMSEA; CFI, Comparative Fit Index.

Table 2.5. Descriptive statistics, reliability and intercorrelations of the ISI-CT subscales (Study 2).

\begin{tabular}{|c|c|c|c|c|c|c|c|c|c|c|}
\hline & 1 & 2 & 3 & 4 & 5 & 6 & $M$ & $S D$ & $\alpha$ & $\begin{array}{c}\text { Item-total } \\
\text { correlations }\end{array}$ \\
\hline 1. Exploratory Informational & 1 & & & & & & 3.67 & .74 & .65 & $.36-.51$ \\
\hline 2. Analytical Informational & $.30^{* *}$ & 1 & & & & & 4.24 & .59 & .76 & $.49-.62$ \\
\hline 3. Diffuse-avoidant & $-.13^{* *}$ & $-.24^{* *}$ & 1 & & & & 2.69 & .77 & .73 & $.38-.68$ \\
\hline 4. Normative to the Host Society & $.12^{* *}$ & .03 & .09 & 1 & & & 3.12 & .78 & .75 & $.48-.58$ \\
\hline 5. Normative to the Country of Origin & $-.15^{* *}$ & $-.17^{* *}$ & $.28^{* *}$ & .04 & 1 & & 2.52 & .71 & .68 & $.32-.55$ \\
\hline 6. Identity Commitment & -.02 & $.15^{* *}$ & $-.36^{* *}$ & $.16^{* *}$ & $-.20^{* *}$ & 1 & 3.36 & .89 & .82 & $.45-.72$ \\
\hline
\end{tabular}

Note. $* * \mathrm{p}<.01 ; * \mathrm{p}<.05$ 
Figure 2.1. Standardized coefficients for confirmatory factor analysis of the identity style subscales of the ISI-CT (Study 2).

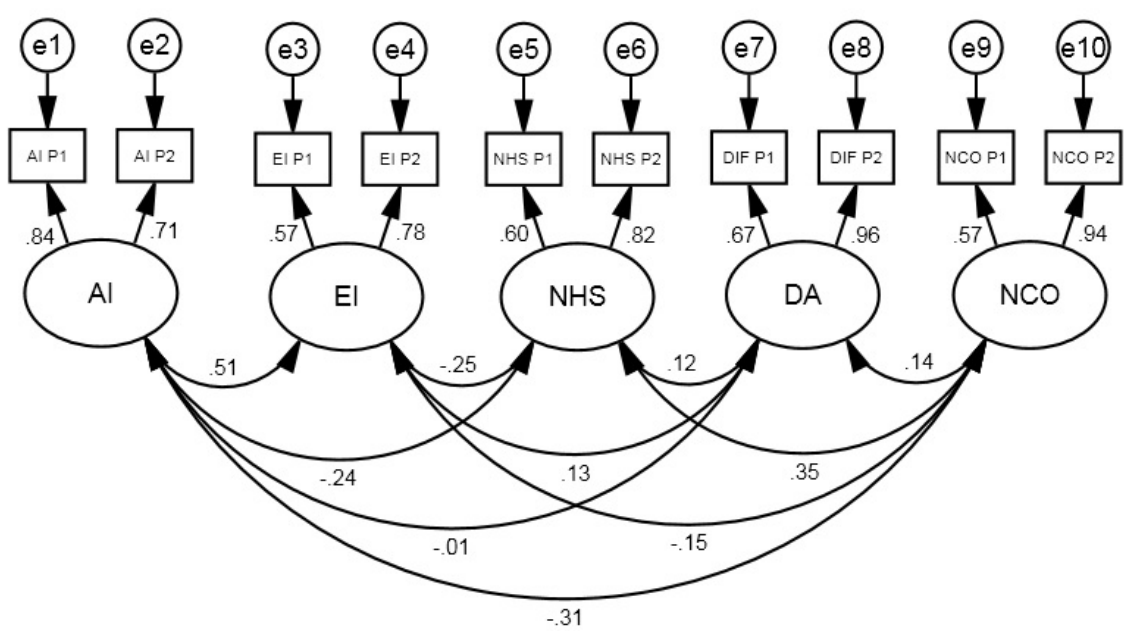

Note. Coefficients are based on StdYX standardization in Mplus. Arrows from circles indicate error or residual variances. All parameters are statistically significant at the 5\% level. AI, Analytical Informational, EI, Exploratory Informational, NHS, Normative to the Host Society, NCO, Normative to the Country of Origin, DA, Diffuse-avoidant.

Figure 2.2. Standardized coefficients for confirmatory factor analysis of the identity commitment subscale of the ISI-CT (Study 2).

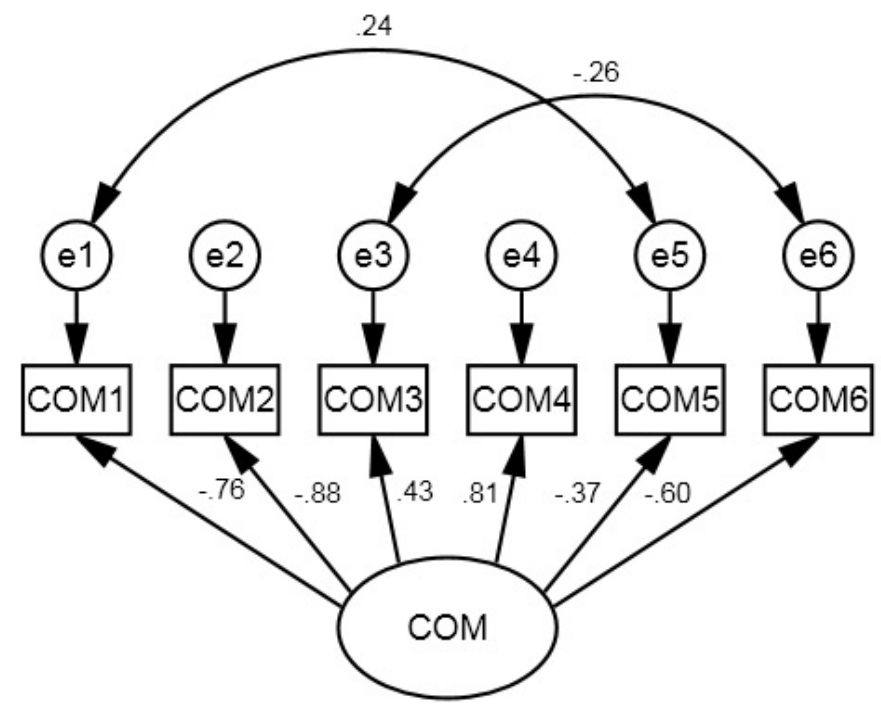

Note. Arrows from circles indicate error or residual variances. All parameters are statistically significant at the $5 \%$ level. Coefficients are based on StdYX standardization in Mplus. COM, Identity Commitment. 


\section{Study 3: Stability over Time}

The aim of our final investigation was to examine longitudinal relationships among the identity styles. Theory suggests that, although most individuals have the cognitive capacity to utilize all of the social-cognitive strategies, there are relatively stable individual differences in the preference and cognitive accessibility of specific identity styles (Berzonsky, 2008). Studies have reported strong test-retest reliability for identity styles after two weeks, indicating that scores on social-cognitive identity strategies are fairly stable in a short-term context, but some research has shown substantially lower correlations after four months (Luyckx, Lens, Smits, \& Goossens, 2010; Smits, 2009) and one year (Duriez, Smits, \& Goossens, 2008). The decline in the correlations over time might indicate a shift in preferences for specific social-cognitive strategies. However, to our knowledge, no studies have modelled temporal changes in the use of identity styles.

The main goal of this study was to investigate whether the adoption of specific identity styles is stable in acculturation and the extent to which cross-lag associations can emerge between strategies over time. Cultural transition presents a context in which changes in identity can happen rapidly and the effectiveness and utility of strategies may be greatly affected by factors related to the receiving environment. Strategies that prove to be effective at the beginning of transition, when immigrants have a relatively narrow social network and are unfamiliar with the cultural norms, might become less so as the elapsed time increases and immigrants gain more experience in the host environment. It is probable that moderate levels of fluctuation in the preferred social-cognitive orientation might be necessary for longterm adaptation.

We were specifically interested in the temporal relationship between the two types of informational styles. The exploratory and analytical informational styles emerged as distinctive factors of information processing and decision making. However, the findings indicated they are weakly associated. Based on the nature of strategies and behaviours involved in exploration, we propose that the separation of the two styles is partly temporal, such that the exploratory behaviour precedes the analytical one. More specifically, we argue that immigrants cannot consider alternatives, unless they have enough information about these alternative options. Therefore, they need to seek information and use more exploratory strategies first in order to be able to analyse their options and make choices. Consequently, we expected that, even though the two styles can function in an independent fashion, the exploratory informational style would predict changes in the analytical informational style over time. 


\section{Method}

\section{Procedure and Participants}

The study employed a six-month longitudinal design with two measuring points.

Participants were invited to complete an online survey. At the end of both surveys,

participants had a chance to go into a lucky draw for Amazon vouchers. We collected contact information for the draw in a way that did not allow us to match email addresses to survey responses. To match the two survey responses, participants were asked to generate a unique code by answering four personal questions (e.g., month of birth or last letter of surname) at each measuring point. The resulting codes were unique and specific for each participant.

At the first measuring point, 280 immigrants completed the survey. The drop-out rate was $51 \%$ by the second wave. Therefore, the final sample consisted of 137 immigrants $(55 \%$ male) with a mean age of $M=30$ years ( $S D=9$ years) and an average length of stay of $M=$ 2.8 years $(S D=3$ years) at the second measuring point. Respondents originated from North America (28.5\%), Western Europe (28.5\%), Eastern Europe (12.4\%), South America (10.9\%), Australia and New Zealand (8\%), East-, South-, Southeast Asia and the Pacific (6.6\%), Africa (3.6\%), and the Middle East (1.5\%). Most participants were residing in Western Europe (41.6\%), which was followed by East-, South- and Southeast Asian countries (23\%), North America (16.8\%), Australia and New Zealand (11.7\%), Eastern Europe (3.6\%), Africa (1.5\%), and South America (1.5\%). The majority of the sample were non-native speakers of the official language of the host country $(82.5 \%)$ and $38.7 \%$ were visible ethnic minorities. Most participants had a working visa (41.6\%), followed by respondents with study visa (27\%), resident visa (21.9\%), dual citizenship (4.4\%), and other types of visa (e.g., family visa, 5.1\%). Attrition analysis indicated that those who remained in the study identified with the immigrant status to a greater extent $(M=3.96, S D=2.10$, on a 7-point scale) compared to their peers who dropped out $(M=3.29, S D=2.02) ; t(277)=-2.72, p=$ .007 , Cohen's $d=.36$. There were no other significant differences between those who did and did not complete the second survey.

\section{Materials}

The ISI-CT and demographic questions were administered as part of a larger survey on psychological adjustment and identity of immigrants. At Time 1, the Cronbach's alpha for AI, EI, DA, NCO, and NHS was .63, .76, .71, .73, .77, and .82. The corresponding values at Time 2 were $.66, .70, .64, .66$, and .87 . 


\section{Statistical Analysis}

Longitudinal relationships among the identity styles were examined by using crosslagged analysis. The cross-lagged model allowed us to determine whether different identity styles predicted each other over time during cultural transition. We started with a fully saturated model by including all direct paths and every possible cross-lagged relationship and then removing non-significant paths.

Figure 2.3. Standardized coefficients for a cross-lagged model testing the temporal stability of the identity styles (Study 3).

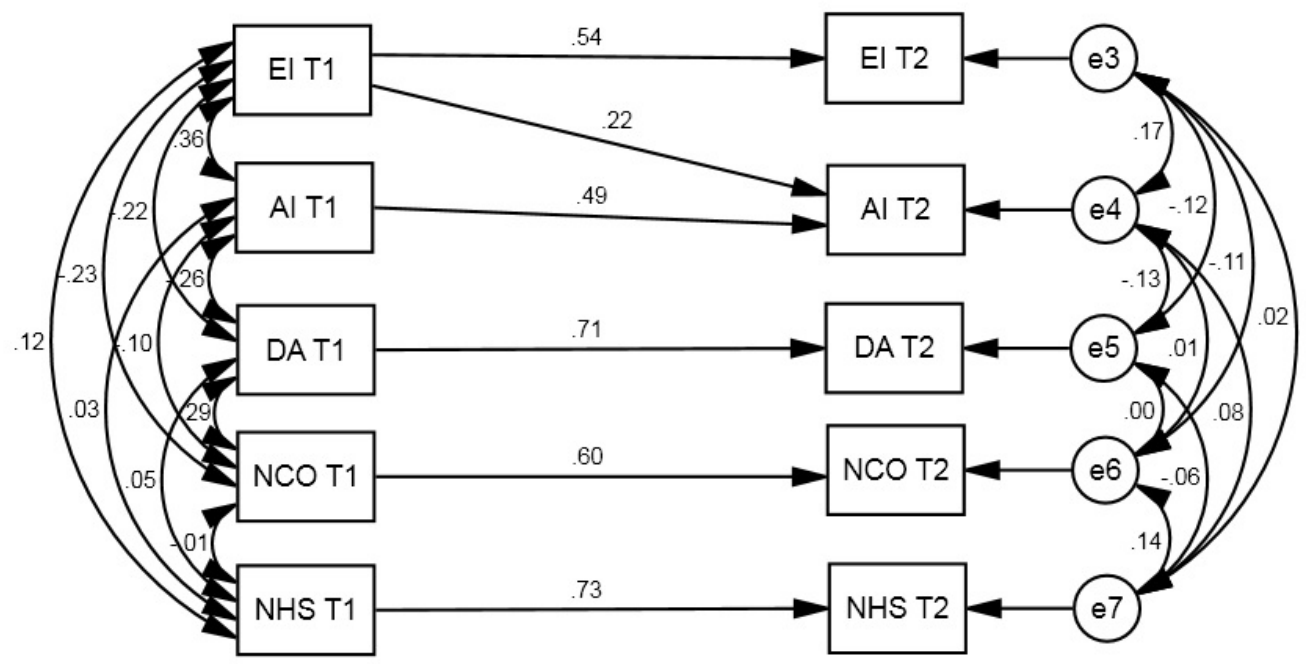

Note. AI, Analytical Informational, EI, Exploratory Informational, NHS, Normative to the Host Society, NCO, Normative to the Country of Origin, DA, Diffuse-avoidant.

\section{Results and Discussion}

The final model revealed strong temporal stability, such that the paths between T1 and $\mathrm{T} 2$ for each scale were significant with regression weights ranging from .49 to .73 (average $=$ .61, Figure 2.3). The final model, not surprisingly, depicted on Figure 2.3 yielded a very good fit to the data $\left(\chi^{2}=10.37, \mathrm{df}=19, \chi^{2} / \mathrm{df}=.55, \mathrm{CFI}=1, \mathrm{NFI}=.976, \mathrm{RMSEA}(90 \% \mathrm{CI})=.000\right.$ $(<.001 ; .013)$, SRMR = .032). Furthermore, one significant cross-lag relationship was identified. In line with our expectations, participants who used more exploratory informational (EI) strategies at T1, reported higher levels of analytical informational processing (AI) at T2 $(\beta=.22, p=.003)$, which supports the temporal primacy of EI over AI. More specifically, our longitudinal findings indicate that when moving to a new society, the culture of which might be largely different from one's heritage culture, immigrants need to 
explore and gain enough information about the norm- and value system of the receiving country, before they can actually consider alternatives and decide what values, norms and rules they want to endorse and incorporate into the self. However, it is important to highlight that the direct links between the $\mathrm{T} 1$ and $\mathrm{T} 2$ variables indicate that the two strategies can function independently as well. No other cross-lagged relationships have emerged. The direct links between T1 and T2 were strong for diffuse-avoidance, NCO and NHS indicating relatively high levels of temporal stability for these strategies.

\section{General Discussion}

The present research was conducted in response to the need for a more processoriented approach to the understanding of identity development during cultural transition (Schwartz et al., 2006). Building on Berzonsky's $(1989,2011)$ theory of social-cognitive identity styles, the main objectives of the series of studies reported in the current paper are to demonstrate that identity styles are in operation in acculturation identity crises, and to provide a survey instrument to assess social-cognitive information processing strategies and identity

styles in culturally diverse immigrant populations. Although social-cognitive identity processing styles have been intensively researched with the general population with respect to normative, developmentally induced identity crises affecting personal identity, no research has previously extended the scope of investigation on social identity aspects to individuals experiencing normative acculturation identity crises evoked by situational-contextual changes.

Five identity styles were initially identified that mirror the structure of the original identity style inventory, i.e., informational (analytical and exploratory), normative (heritage vs. host), and diffuse-avoidant styles. The five-factor structure of the ISI-CT was confirmed in Study 2, and all styles demonstrated acceptable construct, convergent and discriminant validity. The distinction between NCO and NHS suggests that immigrants confront two, often fundamentally different, value- and norm systems during cultural transition. Findings suggested that being normative to the receiving society and the country of origin comprise independent orientations to problem-solving and information processing in a cross-cultural context. Furthermore, analytical (AI) and exploratory informational (EI) styles emerged as distinctive components of social-cognitive processing. Our research results indicated that the two styles have similar cognitive-motivational basis; however, only AI was significantly related to immigrant commitment. In addition, longitudinal findings suggested the temporal primacy of EI in relation to AI. 


\section{Limitations, Future Directions and Concluding Comments}

Although the psychometric analyses of the ISI-CT were promising, the study is not without its limitations. This paper is the first step to adapt a measure of identity styles for the immigrant population, and therefore it was exploratory in nature. The results need to be replicated and further tested with samples of immigrant populations in several host environments. Notwithstanding that supporting evidence was provided for the construct validity of the instrument, its predictive and incremental validity were not tested. Another limitation is that the broader socio-cultural characteristics of the receiving environments and the countries of origin were not investigated. However, it is fair to assume that the use and effectiveness of strategic information processing styles will be influenced by social and cultural factors. As partly suggested by our findings, immigrants who experience greater levels of cultural distance or perceive themselves visibly different from the majority group might find specific strategies more effective in dealing with acculturative identity stress. Lastly, cultural group differences were not examined. Although we controlled for cultural background during the validation process, our sample sizes did not allow us to use measurement equivalence tests or related comparisons across cultural groups.

Although the issues discussed above could not be properly addressed in the present paper, they provide potential avenues for future research. Identity reconstruction occurs in response to the wider cultural and social setting; therefore, future studies should take contextual factors into account when examining the processes involved in identity development. Subsequent investigations could also apply complex model testing strategies to determine the power of acculturation identity styles in predicting both positive and negative identity outcomes and to explore their differential impacts on the psychological and sociocultural adjustment of immigrants. The findings of our third study highlight the importance of longitudinal research in the understanding of identity processes and how they influence identity development in cultural transition.

In conclusion, our findings suggest that immigrants apply the same set of general social-cognitive strategies in response to identity stress, regardless whether it is evoked by situational or developmental changes. Furthermore, our analyses indicate that the Identity Style Inventory - Cultural Transition Version is a reliable and valid measure to assess socialcognitive information processing strategies in cultural transition. It is the first validated assessment tool designed for the immigrant population, which allows for a more comprehensive analysis of processes underlying identity reconstruction during acculturation. 


\section{Footnote}

1. ISI-5 (Berzonsky et al., 2013) was published after the completion of study 1 and 2. 


\title{
Chapter 3
}

\section{Identity development during cultural transition: The role of social-cognitive identity processes}

\author{
Agnes Szabo \& Colleen Ward
}

Victoria University of Wellington

Manuscript published

Szabo, A., \& Ward, C. (2015). Identity development during cultural transition: The role of social-cognitive identity processes. International Journal of Intercultural Relations, 46, 13-25. 


\begin{abstract}
Based on fundamental cognitive differences, Berzonsky (1989) proposed three different strategies of exploration behaviour that underlie the process of identity development: informational, normative, and diffuse-avoidant styles. The present study extends Berzonsky's theorizing to acculturation research and examines the social-cognitive processes underlying identity development of new immigrants $(N=218)$. We investigate informational (analytical and exploratory), normative (heritage and host) and diffuse-avoidant identity styles as predictors of identity commitment and identity outcomes (self-esteem, self-concept clarity and ethno-cultural identity conflict). Two positive and two negative pathways to identity outcomes were identified, which were fully or partially mediated by identity commitment. Analytical informational style and normative orientation to the host society predicted stronger identity commitment, which, in turn, resulted in more positive identity outcomes. In contrast, diffuse-avoidant and exploratory informational styles exerted negative effects on identity outcomes via identity commitment. Furthermore, identity commitment buffered the negative impact of normative orientation to the country of origin on self-esteem. Taken together our findings indicate that identity commitment plays a central role in identity reconstruction of new immigrants.
\end{abstract}




\section{Introduction}

Identity is strongly intertwined with socio-cultural factors, such as language, societal norms, history, and belief systems. Negotiating or living in-between two cultures/societies can be psychologically and socio-culturally challenging, and as a result immigrants frequently experience normative acculturation identity crises, including ethno-cultural identity conflict, which has a detrimental effect on the psychological (Ward et al., 2011) and socio-cultural (Leong \& Ward, 2000) adjustment of acculturating individuals. In contrast, a coherent identity can successfully guide the behaviour of immigrants in their everyday life and buffer the harmful effects of negative life events (S. J. Schwartz, 2005; S. J. Schwartz et al., 2006). In a highly globalized world, where more than 200 million people are living as international migrants (OECD, 2013), continuously confronting and negotiating the demands of at least two different cultures, there is a growing need to understand how cultural transition affects the identity of immigrants and explore the factors that can potentially contribute to the development of a positive and coherent immigrant identity. So far however, research has mainly focused on differences in the structure of immigrants' identity, i.e., the relationship among various social-cultural identity aspects (e.g., blended vs. separated), but little is known about the mechanisms underlying identity development of adult immigrants during acculturation (Leong \& Ward, 2000; Ward, 2008). In this paper, we argue that to gain a deeper insight in to the processes involved in finding a cultural home and creating a bicultural/multicultural identity over time, it is crucial to understand the dynamics of identity formation during cultural transition from the very early stages of acculturation.

Processes involved in acculturation have often been explained by using theories originating from other areas of psychology, such as the stress and coping framework to understand acculturative stress (Ward et al., 2001), social identity theory to investigate intergroup relations in cultural transition (Kosmitzki, 1996), or the theory of social cognition to elucidate cultural frame-switching (Hong, Morris, Chiu, \& Benet-Martinez, 2000). One approach that, we believe, deserves more attention in the acculturation literature is developmental theory. More specifically, we will argue that Erikson's $(1950,1968)$ psychosocial theory of identity development provides a potential framework to understand the mechanisms involved in identity reconstruction during acculturation.

\section{Psychosocial Theory of Identity Development}

Identity has been the focus of interest in almost all areas of psychology, and each field has conceptualized the construct somewhat differently depending on the scope of investigation. Before discussing how Erikson's $(1950,1968)$ psychosocial theory of identity 
development can be applied to test identity processes in acculturating individuals, it is briefly described how identity has been conceptualized in the present research.

From a social-developmental point of view identity can be defined as a self-regulatory social-psychological construct that directs attention and influences information processes and behaviour. Identity helps us understand who we are, creates meaning and commitments, forms coherence and harmony between goals, beliefs and values, and fosters a sense of personal control and the ability to recognize future possibilities (Adams \& Marshall, 1996, p. 433). At the same time it is deeply embedded in a social-cultural context, the change of which potentially shapes identity and encourages people to continuously reorganize their selfdefinitions. In the present research identity is defined as a framework for self-definition that incorporates both personal and social aspects of identity. In this study we do not focus on the structure of identity or the relationships among specific identity aspects, but rather investigate identity outcomes. In our terminology, identity outcomes refer to the perception and evaluation of identity. For example, we argue that an individual who has a clear and consistent sense of identity and how its components are inter-related will be characterized by a positive/balanced/achieved/consolidated identity.

According to Erikson's $(1950,1968)$ psychosocial theory, the reconstruction of identity is facilitated by disequilibrium in identity that is often defined as an identity crisis. Identity crises are normative periods of identity development that occur when people actively examine and question identity issues, such as beliefs, aspirations and commitments to specific values. Marcia (1966) identified two factors - exploration behaviour and commitments - that underpin identity development. He has argued that when people experience a normative crisis over identity, they not only actively explore their environments and the possible alternatives, but also must be willing to commit themselves to specific values in order to achieve identity development. More specifically, identity achievement is reached via active exploration that moves to strong and stable commitments. Although Marcia (1966) identified the underlying components of identity development and described achievement as a possible outcome in detail, his theory has been widely criticized, because it does not address the process of reconstruction, i.e., how and why individuals become identity achievers.

\section{Identity Styles: Social-cognitive Processes of Identity Development}

The theory of identity styles developed by Berzonsky (1989) aims to explore how people deal with problems, make decisions and form commitments when they actively examine identity issues. It is a process-oriented approach to identity development that builds on the psychosocial theory of identity (Erikson, 1950, 1968) and addresses the critiques about 
Marcia's (1966) identity status paradigm. Berzonsky (1989) has argued that when people undergo an identity crisis, differences can be observed in their social-cognitive processes; they solve problems and explore their environment in distinctive ways. Based on fundamental cognitive differences, Berzonsky (1989) proposed three different strategic styles of exploration behaviour (i.e., the tasks involved in creating, maintaining or reconstructing one's identity) that underlie the process of identity development: informational style, normative style and diffuse-avoidant style. It has been shown that people have the cognitive ability to utilize all of these social-cognitive strategic styles (Berzonsky \& Ferrari, 1996; Berzonsky \& Kuk, 2005), so that self-reported individual differences derive from a dominant orientation to information processing and problem solving strategies rather than from developmental differences in social and cognitive capacity (Berzonsky, 2008).

Individuals who process on an informational basis are more effective in many social and cognitive areas (e.g. cognitive complexity, cognitive motivation, thinking style, decision making, coping). They are open to explore alternatives as well as to actively seek and evaluate self-relevant information. They are motivated by perceived discrepancies between their own and others' views (Berzonsky, 1989, 2008). People who score highly on a measure of informational style are also willing to engage in identity revision, and they are usually characterized by a well-differentiated and well-integrated identity structure that is anchored in personal self-esteem (Berzonsky et al., 2003).

Normative individuals report a high level of self-control, intolerance for ambiguity, need for closure, and resistance to change (Duriez \& Soenens, 2006). Their behaviour is directed by social norms, order, traditions, obligations, and the enhancement of stability (Berzonsky, Cieciuch, Duriez, \& Soenens, 2011). Perceived discrepancies between their own values and the values of significant others often result in frustration and anxiety; hence, they try to maintain their self-views through an automatic internalization of norms, rules and value systems (Berzonsky, 1989). A normative style is effective in familiar environments where goals and values of the person and those of the institutions do not differ and conflict.

Strategic avoidance of negative self-relevant feedback and procrastination are the key components of the diffuse-avoidant style (Berzonsky \& Ferrari, 2009). Diffuse-avoidant people typically delay exploration of alternatives and decision making as long as possible; therefore, they make short-term decisions and often use pro-decisional rationalization as well as excuse-making (Berzonsky \& Ferrari, 2009). Because of weak commitments the diffuse identity style concatenates with a loosely integrated and present-oriented identity structure (Berzonsky et al., 2003) as well as with a diffuse identity (Berzonsky, 1989). 
A growing body of research has shown that identity formation styles lead to differences not only in the structure, but also in the evaluation (i.e., affective elements) and perception (i.e., cognitive elements) of identity. Although research findings are not completely conclusive, studies converge to link the informational and normative styles to higher levels of self-esteem (Crocetti et al., 2009; Eryigit \& Kerpelman, 2009; Nurmi, Berzonsky, Tammi, \& Kinney, 1997; Soenens, Berzonsky, Dunkel, Papini, \& Vansteenkiste, 2011), self-concept clarity (Eryigit \& Kerpelman, 2009; Nurmi et al., 1997), self-acceptance (Crocetti \& Shokri, 2010), identity integration (Luyckx et al., 2007) and identity balance (Beaumont \& Pratt, 2011). In contrast, diffuse-avoidance has been associated with increments in identity distress (Beaumont \& Scammell, 2012) and decreased levels of self-esteem (Crocetti et al., 2009; Nurmi et al., 1997; Passmore, Fogarty, Bourke, \& Baker-Evans, 2005; Soenens et al., 2011), self-concept clarity (Johnson \& Nozick, 2011; Nurmi et al., 1997), selfacceptance (Vleioras \& Bosma, 2005), identity balance (Beaumont \& Pratt, 2011), and identity integration (Luyckx et al., 2007). Although the links between identity styles and a variety of identity outcomes have been established, the magnitude of the correlations ranges between .10 and .37 indicating only weak to moderate associations. On the other hand, in the abovementioned investigations, identity commitment has revealed moderate to strong correlations with both identity styles and identity outcomes. In addition, a meta-analysis by Bosch and Card (2012) has shown that identity styles are moderate to strong predictors of identity commitment.

These findings suggest that the three sets of variables (i.e., identity styles, identity commitment and identity outcomes) are strongly interrelated and that, even though identity styles consistently show a direct effect on identity outcomes, the ability to make commitments plays a stronger role in predicting a positive and coherent identity. Berzonsky (2003) proposed three conceptual frameworks to investigate the relationship among identity styles, identity commitment and various outcome variables. The direct-effects model suggests that identity styles and identity commitment show a direct and independent link to outcome variables. The moderational-effects model postulates that the direct associations between identity styles and the dependent measures can either be exacerbated or buffered by identity commitment. According to the mediational-effects model, the relationship between identity styles and outcome variables can be mediated by identity commitment.

The majority of studies have examined direct effects between identity styles and identity outcomes, but there is also evidence that identity commitment mediates the influence of identity styles on outcomes such as personal agency and self-acceptance (Berzonsky, 2003; 
Crocetti \& Shokri, 2010). These findings suggest that the mediational approach might be the key to understanding how identity styles contribute to the development of a coherent, balanced and positively evaluated identity. According to Marcia (1966), making strong commitments is a prerequisite to achieve a new identity; therefore, it is expected that identity commitment serves as a mediator, through which identity styles exert positive or negative effects on identity outcomes.

\section{Identity Conflict and Acculturation}

While Erikson's (1968) definition implies that identity crises are evoked by developmental changes, Baumeister et al. (1985) discussed that identity crises can be caused by situational changes and play out as identity conflicts. They argued that identity conflict derives from the pressure to make decisions in situations when existing commitments are perceived as incompatible or conflicting. More specifically, an identity conflict develops if two established and so far compatible commitments become conflicting due to changed situational factors or contradictory expectations of the social environment. Baumeister et al. (1985) suggested that immigrants would be a potential group to test the theory of identity conflict empirically, because identity crises are common in connection with acculturation, and immigrants often confront contradictory demands from the heritage and host cultures.

The theory of identity styles provides a potential framework to explain identity development in cultural transition. Based on Berzonsky's $(1989,1992)$ work,Szabo, Ward, and Fletcher (2015) have developed a new version of the Identity Style Inventory to test whether identity styles are in operation in a situation-induced identity crisis. They have identified a diffuse-avoidant style and two distinct types of informational (analytical and exploratory) and normative (host and heritage) processing during cultural transition. More specifically, it has been demonstrated that the normative style can play out in two different ways during cultural transition: One can either be normative toward the host society or have a normative orientation to the country of origin.

Although Szabo et al.'s (2015) research has shown that immigrants use similar socialcognitive strategies to deal with a situation-induced identity crises as have been identified with the general population in periods of normative identity crises, no studies have been previously conducted to explore how these different social-cognitive strategies affect the identity reconstruction of immigrants and whether the relationship between identity styles and identity outcomes is mediated by identity commitment. 


\section{The Present Study}

Migration affects aspects of both personal and social identity, and challenges the basis of self-definition. As a result of social-cultural transition, a comprehensive structural change occurs in the identity of the acculturating individual, which can result in an identity crisis (Baumeister et al., 1985). In a new social context identity issues and the differences between the cultural and societal expectations of the original and the host country become salient and activate identity processes.

Figure 3.1. Proposed model of immigrant identity development in cultural transition.

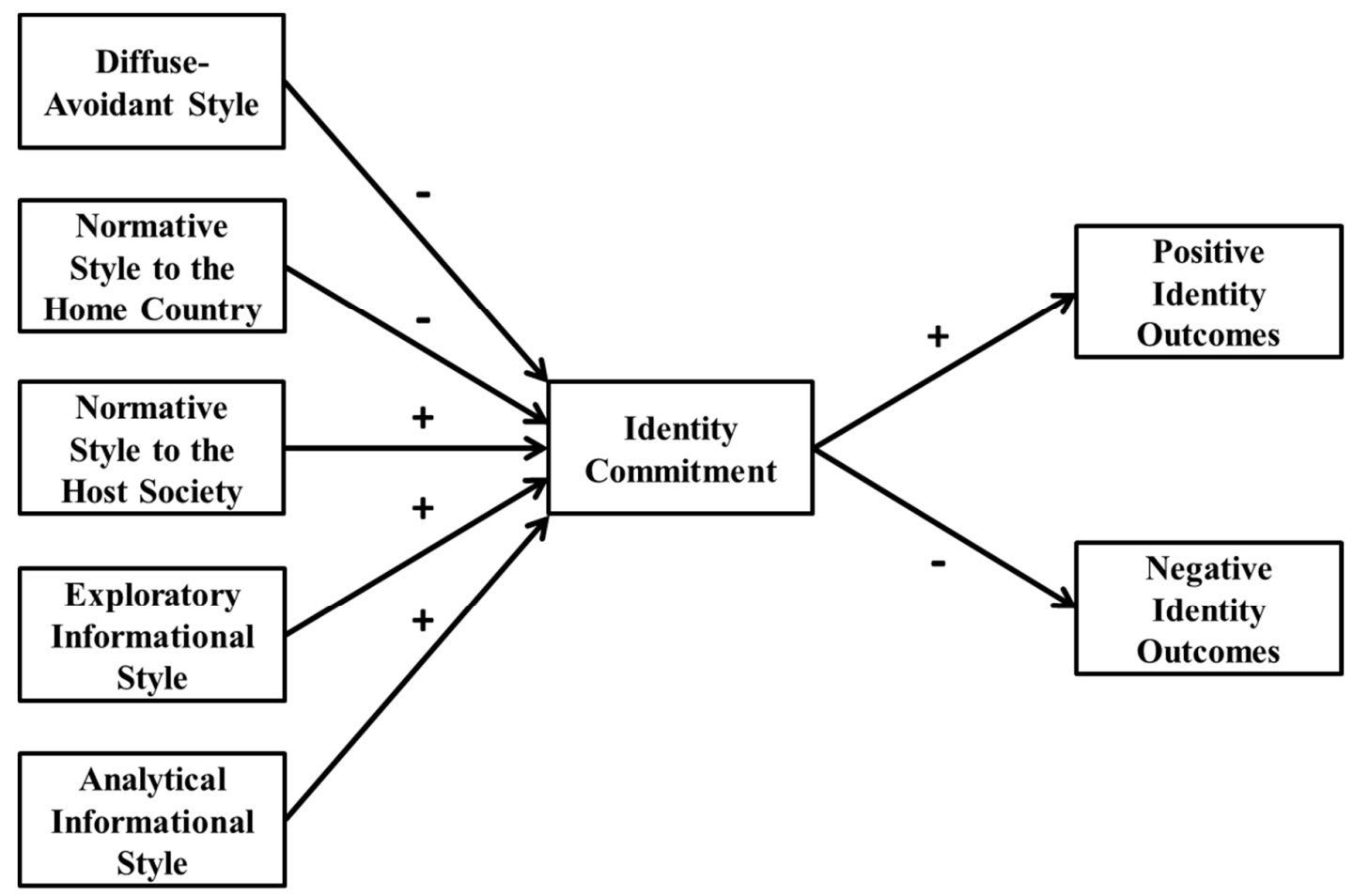

The present research is designed to gain a better understanding of identity processes of acculturating individuals and to explore how different cognitive strategies can facilitate positive identity development in a cross-cultural context. A mediational model is proposed, in which identity commitment mediates the link between identity processing styles and identity outcomes. More specifically, it is hypothesized that informational styles and normative orientation to the host society will predict higher levels of identity commitment (H1/A), which in turn will lead to increased self-esteem and self-concept clarity (H1/B), and decreased ethno-cultural identity conflict (H1/C). In contrast, normative orientation to the 
host society and diffuse-avoidance are hypothesized to be associated with lower levels of identity commitment (H2/A), which in turn will lead to more negative evaluations of the identity (i.e., decreased self-esteem and self-concept clarity; H2/B) and higher levels of ethno-cultural identity conflict (H2/C, see Figure 3.1).

The focus of our investigation is on recent immigrants, who have been living in the host society for no longer than one year. Due to increased levels of both intragroup (i.e., with other immigrants) and intergroup (i.e., with host nationals) contact (Sussman, 2000; Turner, Oakes, Haslam, \& Mcgarty, 1994), identity issues are more salient during earlier stages of acculturation, and perceived differences in values, norms and rules are likely to be intensified and excessive. In addition, we aim to capture the period when identity formation strategies potentially show the highest variability and influence. Therefore, we decided to constrain our sample to newly arrived immigrants.

\section{Method}

\section{Procedure and design}

A subject variable design was employed for this study, with the variables of interest being that of identity styles during cultural transition and identity commitment, self-esteem, self-concept clarity, and ethno-cultural identity conflict. Participants were invited to complete an anonymous online survey on immigrant identity through social media and various immigrant organizations and networks. The survey was administered in English. At the end of the survey, participants had the chance to go into a lucky draw for Amazon vouchers. Respondents, who wanted to go into the draw, were redirected to a website unrelated to the survey, where they could provide their contact details. We collected this information in a way that did not allow us to match the participants' contact details to their survey responses.

\section{Sample}

The sample included 218 recent immigrants who had resided in the host society no longer than one year $(M=7.56$ months, $S D=4.03$ months $)$ at the time of data collection. Participants (48.6\% male) ranged in age from 17 to $66(M=27.67$ years, $S D=7.65$ years). The vast majority of respondents $(72.5 \%)$ were non-native speakers of the official language of the country of residence. They also had a high level of proficiency in English, the survey language, with a mean score of $7.2(S D=1.06$, on an 8 -point scale anchored by $1=$ poor, $7=$ excellent, and $8=$ native speaker). More than half of the sample (54.1\%) reported a previous sojourning experience.

The sample included respondents originating from North America (37.2\%), Western Europe (24.8\%), Eastern Europe (17\%), Australia and New Zealand (6.4\%), South America 
(6.4\%), South-, East-, and Southeast Asia (4.6\%), Africa (1.8\%), and the Middle East (1.8\%). Most participants were residing in a Western European country (41.7\%) followed by immigrants living in South-, East-, and Southeast Asia (21.6\%), Australia and New Zealand (11.9\%), North America (9.6\%), Eastern Europe (6\%), South America (4.6\%), Africa (3.2\%), and the Middle East (1.4\%). When asked if they considered themselves to be members of visible ethnic minorities in their country of residence, $37.2 \%$ of the sample replied "yes."

\section{Materials}

The current study was part of a larger survey about the factors contributing to immigrant identity. The survey included questions pertaining to demographic information, the modified version of the Identity Style Inventory, and various measures of identity outcomes.

\section{Identity Style Inventory - Cultural Transition Version (ISI-CT)}

Identity processing styles and commitment were measured by a modified version of the Identity Style Inventory (Berzonsky, 1989). The ISI-CT was specifically developed to assess information processing and decision making strategies as well as identity commitment of acculturating individuals from diverse ethnic and cultural backgrounds (Szabo, Ward, \& Fletcher, 2015). Identity styles capture five distinct social-cognitive orientations to problem solving and information processing during cultural transition, whereas the identity commitment scale assesses clarity in values, strength of adherence to the host society, and certainty in the decision to move abroad. The ISI-CT consists of 28 items that measure these constructs. The items were prefaced by the following instruction: "Please, think about the difficulties that you have to face in the country where you currently live. Read each statement carefully and judge the extent to which it describes you and your behaviour during crosscultural transition. Circle the number that indicates the extent to which you think the statement represents your views and your feelings," and participants were asked to give responses on a 5 -point scale $(1=$ not like me at all and $5=$ very much like me $)$. The ISI-CT includes two subscales assessing informational style: Analytical Informational Style (AI, "When facing a decision, I take into account different points of view before making a choice.”) and Exploratory Informational Style (EI, "I've spent a good deal of time reading and talking to others about the values and rules of the country in which I currently live."), each measured by four items. Similarly, the normative style is captured by two separate subscales: Normative Orientation to the Country of Origin (NCO, "I prefer to deal with situations according to the social norms and standards of my home country.") and Normative Orientation to the Host Society (NHS, "I prefer to deal with situations according to the social 
norms and standards of the country in which I live."), measured by five and four items, respectively. Finally, the ISI-CT contains one scale assessing the Diffuse-avoidant Style (DA, "I try not to think about or deal with problems as long as I can." - measured by five items) and a separate measure for identity commitment in the context of the host society (COM, "I know what I want to do with my future in this country." - measured by six items). During the validation process, the scales revealed acceptable internal consistency, with alphas ranging from .60 to .87 across four samples (Szabo et al., 2014). In this study, all subscales yielded good reliability (NHS: $\alpha=.72$, NCO: $\alpha=.70$, AI: $\alpha=.76$, EI: $\alpha=.60$, DA: $\alpha=.72$, COM: $\alpha$ $=.80)$. Although the EI style showed somewhat lower internal consistency, the Cronbach's alpha is a function of the number of items a scale contains, therefore it is not necessarily a reliable measure of consistency for measures consisting of a few items (Nunnally, 1978). Furthermore, the item-total correlations (ranging from $r=.24$ to $r=.51$ ) and the average inter-item correlation $(r=.26)$ were in the expected interval indicating acceptable internal consistency (Clark \& Watson, 1995; Nunnally \& Bernstein, 1994).

\section{Self-esteem}

Participants completed the Rosenberg Self-esteem Scale (SE) that was designed to assess global self-esteem, individuals' feelings of self-worth and evaluative attitudes toward the self (Rosenberg, 1965). The scale includes 10 items with five statements being reversedkeyed. Sample items are "On the whole, I am satisfied with myself" and "At times, I think I am no good at all" (reversed). The measure was rated on a 5-point scale ranging from $1=$ Strongly Disagree to 5 = Strongly Agree with higher scores indicating higher levels of self-

esteem. Previous studies have reported reliability coefficients for SE ranging from .72 to .82 (Gray-Little, Williams, \& Hancock, 1997). In the present sample, the scale revealed acceptable reliability $(\alpha=.89)$.

\section{Self-concept clarity}

The Self-concept Clarity Scale (SCC) was designed to measure individuals' beliefs about the clarity, consistency and stability of the self (J. D. Campbell et al., 1996). The instrument is a composition of twelve positively and negatively phrased items measuring aspects of consistency and stability of self-beliefs, generic self-certainty, goal-directedness, and decisiveness. Participants gave responses to statements, such as "Sometimes I feel that I am not really the person that I appear to be" (reversed) or "In general, I have a clear sense of who I am and what I am," on a 5-point scale (anchored by $1=$ Strongly Disagree and $5=$ Strongly Agree). During the validation process, the scale yielded acceptable levels of 
reliability (average $\alpha=.86$ ), and it demonstrated good internal consistency $(\alpha=.90)$ in the present study as well.

\section{Ethno-cultural identity conflict}

The Ethno-cultural Identity Conflict Scale (EIC) was developed and validated by Ward et al. (2011) to measure identity conflict experienced by individuals confronting acculturative demands. The 20-item measure includes both negatively and positively phrased items (e.g., "I feel confident moving between cultures" and "I sometimes do not know where I belong") that are rated on a 5-point scale (anchored of $1=$ Strongly Disagree and $5=$ Strongly Agree). Participants who report higher scores on the scale experience higher levels of ethno-cultural identity conflict. Ward et al.'s (2011) validation studies revealed high levels of internal consistency for the EIC (alphas ranged from .89 to .92 across three samples). In the present investigation the scale was also highly reliable $(\alpha=.92)$.

\section{Statistical Analyses}

First, preliminary analyses were conducted to investigate differences between males and females, visible and non-visible ethnic minorities, and native and non-native speakers in the use of identity styles, levels of identity commitment, SE, SCC, and EIC. Next, bivariate correlations were calculated to examine the interrelationship of the variables of interest, as well as their associations with demographic variables, including age and length of stay. Finally, a path model was applied to test the theoretical model depicted by Figure 3.1. Five indices are used to assess the model fit. Models with a chi square/df ratio lower than 2 (Tabachnick \& Fidell, 2007), a CFI and NFI value higher than .95, RMSEA lower than .06, and SRMR lower than .08 were considered to indicate acceptable fit to the data ( $\mathrm{Hu} \&$ Bentler, 1999).

\section{Results}

\section{Preliminary Analyses}

\section{Group comparisons}

Independent samples t-tests were conducted to test group differences in identity processing styles. There was no significant difference between males and females in any of the identity styles. Similarly, no differences were found between native and non-native speakers of the official language of the country of residence. Visible ethnic minorities $(M=$ $3.79, S D=.64)$, however, scored significantly higher in the use of the exploratory informational style compared to non-visible ethnic minorities $(M=3.58, S D=.71), t(216)=$ $2.22, p=.027$, Cohen's $d=.30$. 
Table 3.1. Descriptive statistics and correlations among the variables of interest.

\begin{tabular}{|c|c|c|c|c|c|c|c|c|c|c|c|c|c|c|c|}
\hline & $M$ & $S D$ & $\alpha$ & 1 & 2 & 3 & 4 & 5 & 6 & 7 & 8 & 9 & 10 & 11 & 12 \\
\hline 1. AI & 4.28 & .59 & .76 & 1 & & & & & & & & & & & \\
\hline 2. EI & 3.66 & .69 & .60 & $.29^{* * *}$ & 1 & & & & & & & & & & \\
\hline 3. DA & 2.64 & .75 & .72 & $-.26^{* *}$ & -.10 & 1 & & & & & & & & & \\
\hline 4. NHS & 3.10 & .73 & .72 & .08 & $.15^{*}$ & .12 & 1 & & & & & & & & \\
\hline 5. $\mathrm{NCO}$ & 2.50 & .72 & .70 & -.08 & -.06 & $.31^{* *}$ & .08 & 1 & & & & & & & \\
\hline 6. COM & 3.37 & .85 & .80 & $.19^{* *}$ & -.06 & $-.35^{* *}$ & $.18^{* *}$ & $-.15^{*}$ & 1 & & & & & & \\
\hline 7. SE & 3.67 & .73 & .89 & $.15^{*}$ & -.12 & $-.43^{* *}$ & -.07 & $-.23^{* *}$ & $.42^{* * *}$ & 1 & & & & & \\
\hline 8. SCC & 3.14 & .81 & .90 & $.18^{*}$ & $-.18^{*}$ & $-.48^{* *}$ & -.07 & $-.18^{*}$ & $.53^{* * *}$ & $.67^{* *}$ & 1 & & & & \\
\hline 9. EIC & 2.36 & .71 & .92 & -.11 & $.29^{* *}$ & $.25^{* *}$ & -.05 & .00 & $-.49^{* *}$ & $-.52^{* *}$ & $-.67^{* *}$ & 1 & & & \\
\hline 10. $\mathrm{AGE}^{\mathrm{a}}$ & 27.67 & 7.65 & - & .06 & .07 & $-.25^{* *}$ & -.03 & $-.16^{*}$ & $.21^{* *}$ & $.23^{* *}$ & $.34^{* * *}$ & $-.19^{* *}$ & 1 & & \\
\hline 11. $\operatorname{LOS}^{\mathrm{b}}$ & 7.56 & 4.03 & - & -.11 & .06 & -.06 & -.05 & -.09 & -.12 & -.10 & -.05 & .06 & $.18^{* *}$ & 1 & \\
\hline 12. VEM $^{\mathrm{c}}$ & - & - & - & .01 & $.13^{*}$ & .07 & .00 & -.04 & -.07 & -.05 & $-.16^{*}$ & $.15^{*}$ & .04 & .09 & 1 \\
\hline
\end{tabular}

Note. $* p<.05$ (two tailed), $* * p<.01$ (two tailed). AI = Analytical Informational Style; EI = Exploratory Informational Style, DA = Diffuse-avoidant Style; NHS = Normative Style to the Host Society; NCO = Normative Style to the Country of Origin; COM = Identity Commitment; SE = Self-esteem; SCC = Self-concept Clarity; EIC = Ethno-cultural Identity Conflict; LOS = Length of Stay; VEM = Visible Ethnic Minority $(0=$ no, $1=$ yes $) .{ }^{a}$ Age is measured in years. ${ }^{b}$ Length of stay is measured in months. ${ }^{c}$ Kendall's tau-b correlations are reported with respect to ethnic visibility. 
Three MANOVAs were employed to test group differences in identity commitment and identity outcomes. No significant main effects were found for gender and language proficiency. However, results revealed a significant main effect for visibility, $\lambda=.95, F(4$, 174) $=2.48, p=.045, \eta_{p}{ }^{2}=.05$. The examination of univariate effects revealed a significant difference between visible and non-visible minorities in EIC and SCC, $F(1,177)=5.73, p=$ $.017, \eta_{p}{ }^{2}=.03$ and $F(1,177)=5.85, p=.018, \eta_{p}{ }^{2}=.03$, respectively. More specifically, visible ethnic minorities reported higher levels of EIC $(M=2.51, S D=.69)$ and lower levels of $\operatorname{SCC}(M=2.97, S D=.72)$ than their non-visible counterparts (EIC: $M=2.26, S D=.72$, SCC: $M=3.26, S D=.85)$.

\section{Correlational analysis}

Correlation coefficients, means and standard deviations of the measures are presented in Table 3.1. Bivariate correlations revealed significant relationships between identity commitment and all identity styles, except for EI. AI and NHS positively and weakly related to commitment ( $r=.19, p=.005$ and $r=.18, p=.007$, respectively), while DA and NCO showed small to moderate negative associations with commitment $(r=-.35, p<.001$ and $r=$ $-.15, p=.023$, respectively). In addition, commitment strongly correlated with measures of identity outcomes. More specifically, it had a strong positive relationship with self-esteem ( $r$ $=.42, p<.001)$ and self-concept clarity $(r=.53, p<.001)$ and was negatively associated with ethno-cultural identity conflict $(r=-.49, p<.001)$. Length of stay was unrelated to all measures, whereas age was negatively and weakly related to DA $(r=-.25, p<.001)$, NCO $(r$ $=-.16, p=.018)$ and $\mathrm{EIC}(r=-.19, p=.011)$, while positively associated with $\operatorname{COM}(r=.21$, $p=.002), \operatorname{SE}(r=.23, p=.001)$ and $\operatorname{SCC}(r=.34, p<.001)$.

\section{Model Testing}

The initial path analysis yielded a poor fit to the data. Parameter estimates were examined and results revealed that $\mathrm{NCO}$ was not significantly related to either commitment or the identity outcomes. In addition, modification indices indicated direct links between DA and SE, DA and SCC as well as between EI and EIC. Therefore, we decided to eliminate $\mathrm{NCO}$ from the model and added the direct links between identity styles and the outcome variables. The modified model demonstrated good fit to the data $\left(\chi^{2}=19.93, \mathrm{df}=11, \chi^{2} / \mathrm{df}=\right.$ $1.81, \mathrm{CFI}=.983, \mathrm{NFI}=.963, \mathrm{RMSEA}(90 \% \mathrm{CI})=.061(.008 ; .103), \mathrm{SRMR}=.04$, Figure $3.2)$.

EI $(\beta=-.17, p=.007)$ and DA $(\beta=-.36, p<.001)$ negatively, while $\mathrm{AI}(\beta=.13, p=$ $.050)$ and NHS $(\beta=-.24, p<.001)$ positively predicted commitment. Commitment significantly predicted all measures of identity outcomes and mediated the links between 
identity styles and identity outcomes. More specifically, commitment showed a positive link to $\operatorname{SE}(\beta=.33, p<.001)$ and $\operatorname{SCC}(\beta=.46, p<.001)$ and a negative link to $\operatorname{EIC~}(\beta=-.52, p<$ .001). A full mediation was obtained for NHS and AI (indirect effects are presented in Table 3.2.). In contrast, the links between EI and DA and the identity outcomes were partially mediated by commitment. EI directly predicted EIC $(\beta=.16, p<.001)$ and had an indirect effect on SCC, SE and EIC through commitment. Similarly, DA revealed a direct link to SE $(\beta=-.30, p<.001)$ and SCC $(\beta=-.28, p<.001)$ and indirectly affected EIC, SE and SCC via commitment.

Figure 3.2. Standardized coefficients for the path model testing the proposed theoretical model.

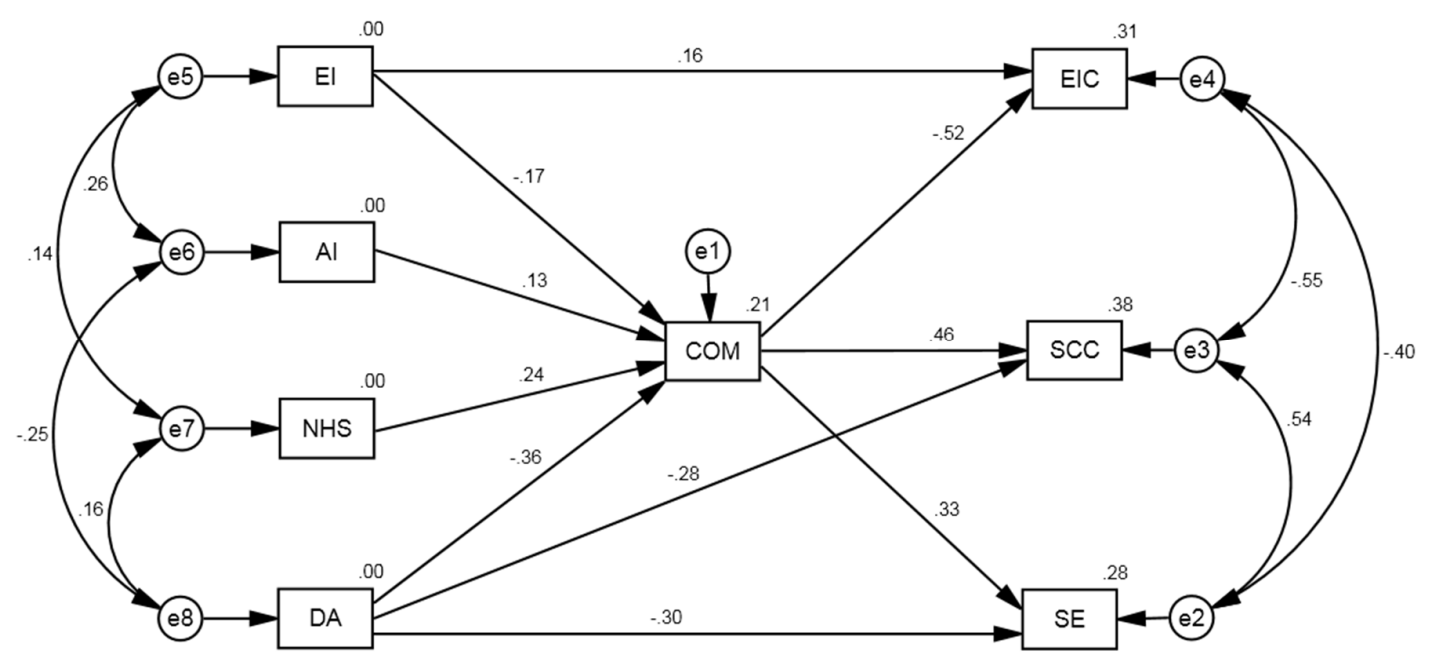

Note. All coefficients are statistically significant at the 5\% level. Fit indices are reported in text. Additional analyses were conducted to test for the potential moderating effect of ethnic visibility. Results revealed that the links among identity styles, identity commitment and identity outcomes were not moderated by ethnic visibility. AI, Analytical Informational Style; EI, Exploratory Informational Style, DA, Diffuse-avoidant Style; NHS, Normative Style to the Host Society; COM, Identity Commitment; SE, Self-esteem; SCC, Self-concept Clarity; EIC, Ethno-cultural Identity Conflict. 
Table 3.2. Indirect effects between identity styles and identity outcomes through identity commitment.

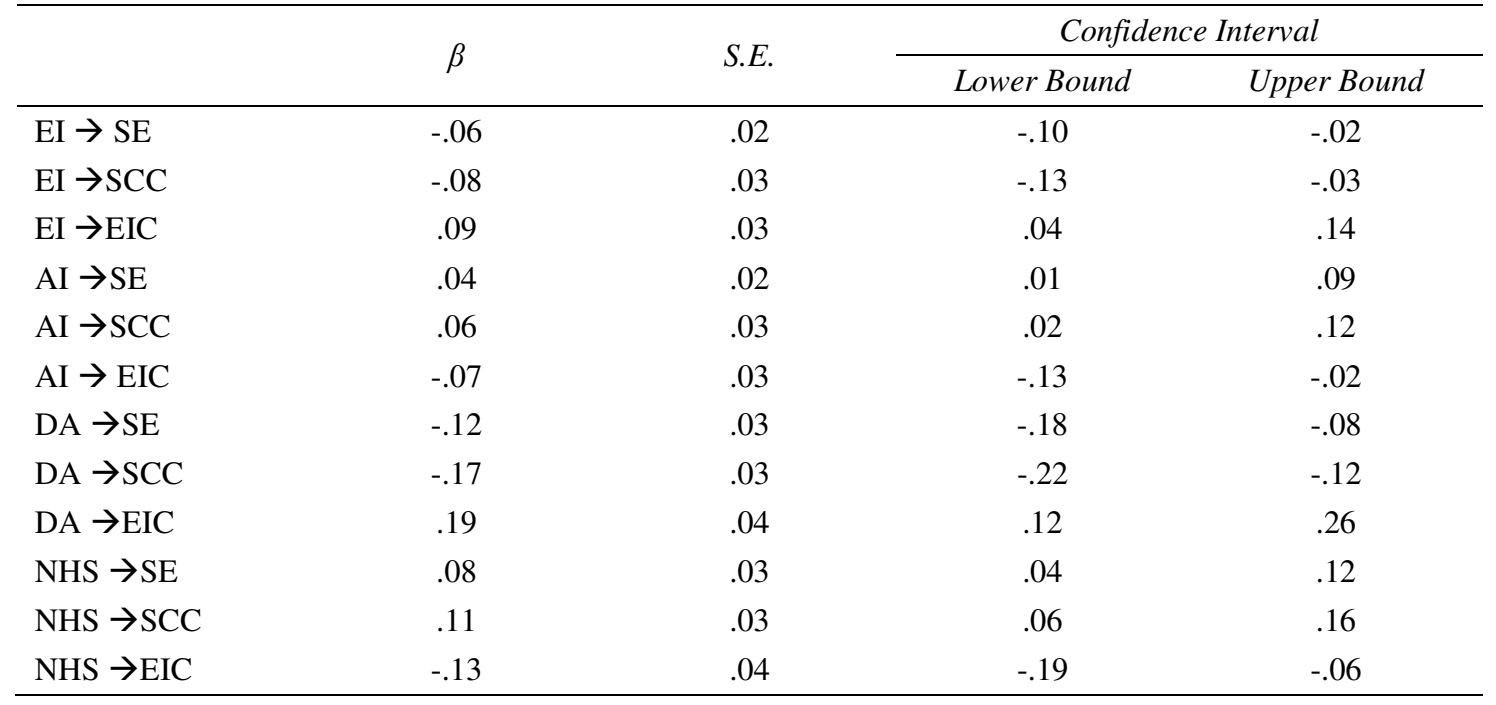

Note. AI, Analytical Informational Style; EI, Exploratory Informational Style, DA, Diffuse-avoidant Style; NHS, Normative Style to the Host Society; NCO, Normative Style to the Country of Origin; COM, Identity Commitment; SE, Self-esteem; SCC, Self-concept Clarity; EIC, Ethno-cultural Identity Conflict.

\section{Additional Analyses}

Additional analyses were applied to further investigate the relationship among NCO, identity commitment and the identity outcomes. Although the path analysis revealed neither mediated nor direct effects between NCO and measures of identity outcomes, the bivariate correlations showed significant associations with SE and SCC. More specifically, NCO was negatively, albeit weakly, related to both SE $(r=-.23, p=.001)$ and $\operatorname{SCC}(r=-.18, p=.016)$. With the path analysis a mediational model was tested, in which commitment was expected to mediate the relationship between identity styles and the outcome variables. However, according to the moderational framework suggested by Berzonsky (2003), it is possible that in the case of NCO commitment functions as a moderator, such that it exacerbates or buffers the impact of $\mathrm{NCO}$ on different outcome variables. To test this alternative hypothesis, three three-step regression analyses were employed with dependent variables being that of SE, SCC and EIC. In the first step AI, EI, NHS and DA were entered to control for the effect of other identity styles. Next, COM and NCO were added to the model to examine their direct impact on identity. Finally, an interaction term between COM and NCO was introduced to test the potential moderating effect.

The models, in which EIC and SCC functioned as dependent variables, revealed no significant interaction effects. However, COM and NCO significantly interacted and 
explained unique amounts of variance in SE above and beyond the direct effects of commitment and the five identity styles (Table 3.3.). The three-step model explained $31.5 \%$ of the total variance in $\mathrm{SE}, F(7,193)=12.69, p<.001$. The four identity styles explained $22.1 \%$ of the variance, which increased by $7.9 \%$ after the introduction of COM and NCO, $F$ $(2,194)=10.88, p<.001$. The interaction term contributed another $1.6 \%$ of variance to the overall model, $F(1,193)=4.41, p=.037$. In the final step, DA $(\beta=-.30, p<.001)$ and $\mathrm{EI}(\beta$ $=-.13, p=.049)$ negatively predicted SE, whereas COM had a positive main effect $(\beta=.29$, $p<.001)$. Furthermore, COM significantly interacted with $\mathrm{NCO}(\beta=.13, p=.037)$ to predict SE. Results obtained were imputed into ModGraph (Jose, 2013) to analyse and display the interaction effect. Post hoc tests revealed that identity commitment significantly buffered the negative effects of NCO on SE (Figure 3.3.). More specifically, NCO was negatively related to SE only under low $(t(198)=-2.63, p=.009)$ and medium $(t(198)=-1.69, p=.092)$ levels of identity commitment. In contrast, NCO did not impact SE under high levels of identity commitment $(t(198)=.06, \mathrm{~ns}$.$) .$

Table 3.3. Hierarchical regression of the prediction of self-esteem.

\begin{tabular}{lllccc}
\hline & & $\mathrm{R}^{2}$ & $\Delta \mathrm{R}^{2}$ & Beta & $\mathrm{t}$ \\
\hline \multirow{4}{*}{ Step 1 } & AI & .22 & $.22^{* *}$ & .09 & 1.32 \\
& DA & & & -.43 & $-6.51^{* *}$ \\
& NHS & & .02 & 0.26 \\
& EI & & -.19 & $-2.81^{* *}$ \\
\hline \multirow{5}{*}{ Step 2 } & AI & .30 & $.08^{* *}$ & .05 & 0.82 \\
& DA & & & -.30 & $-4.22^{* *}$ \\
& NHS & & -.05 & -0.74 \\
& EI & & -.13 & $-2.05^{*}$ \\
& NCO & & -.09 & -1.42 \\
& COM & & .30 & $4.40^{* *}$ \\
\hline \multirow{3}{*}{ Step 3 } & AI & & $.02^{*}$ & .05 & 0.80 \\
& DA & & -.30 & $-4.36^{* *}$ \\
& NHS & EI & & -.06 & -0.92 \\
& NCO & & -.13 & $-1.98^{*}$ \\
& COM & & -.11 & $-1.74^{+}$ \\
& NCOXCOM & & .29 & $4.29^{* *}$ \\
\hline
\end{tabular}

Note. Dependent variable was Self-esteem. ${ }^{* *} \mathrm{p}<.01 ;{ }^{*} \mathrm{p}<.05 ;{ }^{+} \mathrm{p}<.01$. AI, Analytical Informational Style; EI, Exploratory Informational Style, DA, Diffuse-avoidant Style; NHS, Normative Style to the Host Society; NCO, Normative Style to the Country of Origin; COM, Identity Commitment 
Figure 3.3. Identity commitment buffers the negative impact of normative orientation to the country of origin on self-esteem.

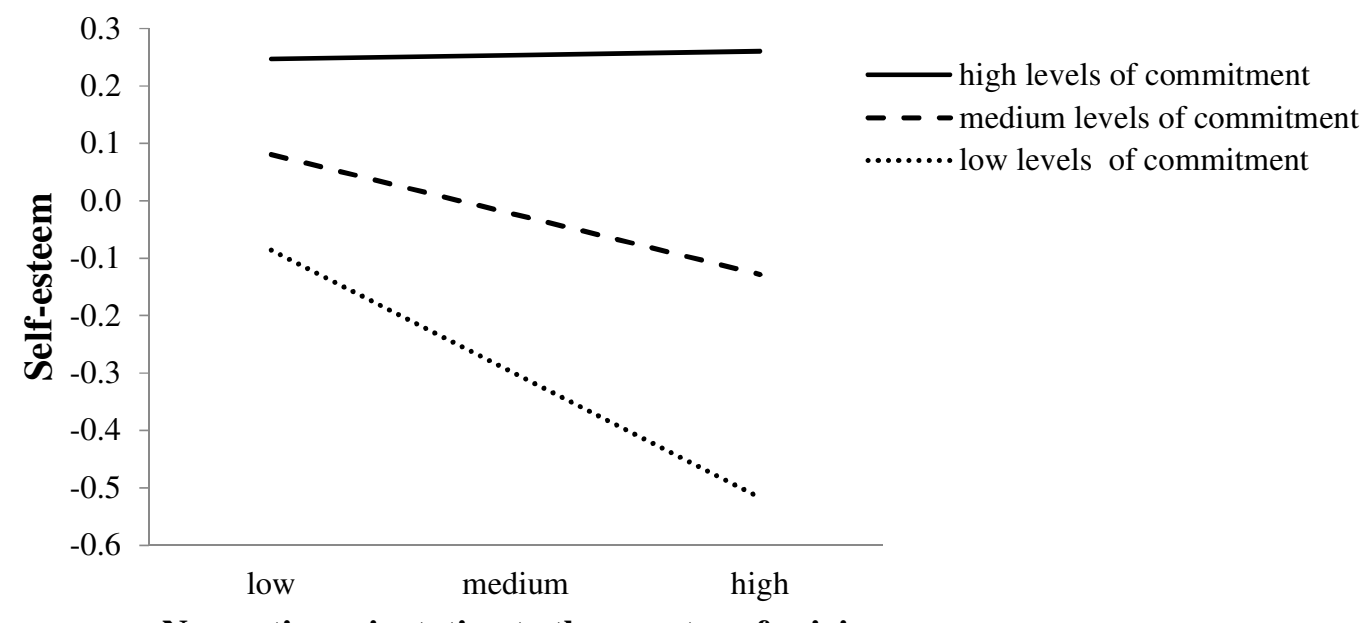

Normative orientation to the country of origin

\section{Discussion}

The present study aimed to investigate the mechanisms underlying identity development in cultural transition and to identify pathways to positive immigrant identity. Findings have shown that identity commitment plays a central role in identity development during acculturation by either mediating or moderating the link between identity styles and a combination of positive and negative identity outcomes. The study has tested five identity styles that capture social-cognitive orientations to decision making and identity exploration in the context of cultural transition (i.e., diffuse-avoidance, exploratory informational, analytical informational, normative orientation to the host society, and normative orientation to the country of origin) and concentrated on three identity outcomes by measuring the evaluation of the identity as a whole (self-esteem), the perception of identity coherence (self-concept clarity) and the experience of conflict over the identity (ethno-cultural identity conflict).

It was hypothesized that the diffuse-avoidant style and the normative style to the country of origin would negatively impact identity outcomes through commitment, whereas the informational styles and the normative style to the host society were expected to serve as positive pathways to immigrant identity. Three out of the five predictions were confirmed; however, findings with respect to the exploratory informational style and the normative style to the country of origin showed a different pattern to what was originally expected. 


\section{Diffuse-avoidant Style}

In line with our hypothesis, the diffuse-avoidant style negatively predicted identity commitment, and thus indirectly led to increased ethno-cultural identity conflict and decreased self-concept clarity and self-esteem. In addition to the indirect effects, diffuseavoidance showed a direct negative link to self-concept clarity and self-esteem. This confirms past research findings with the general population showing that diffuse-avoidance is commonly related to more negative identity outcomes (Beaumont \& Pratt, 2011; Beaumont \& Scammell, 2012; Johnson \& Nozick, 2011; Luyckx et al., 2007; Vleioras \& Bosma, 2005). Previous literature has also shown that those who score higher on diffuse-avoidance experience more problems in many social, emotional and cognitive areas (Adams et al., 2001; Adams et al., 2005; Berzonsky \& Luyckx, 2008; Doumen et al., 2012; Soenens et al., 2005) and are characterized by a diffuse and loosely integrated identity (Berzonsky, 1989, 2003; Berzonsky \& Kuk, 2000; Berzonsky \& Neimeyer, 1994; Berzonsky et al., 2013; Krettenauer, 2005). Similarly, our results suggest that recent immigrants who are processing on a diffuseavoidant basis are at higher risk to develop a conflicted identity and presumably more vulnerable to cross-cultural difficulties.

\section{Informational Styles}

Findings with respect to the informational styles were mixed. Those who score high on the informational style are motivated to consider alternatives and revise their identity, but also willing to make decisions and commitments after critically thinking about their options (Berzonsky, 2008). Consequently, the style has been found to be strongly associated with an achieved identity (Berman et al., 2001; Berzonsky, 1989, 2008; Berzonsky et al., 2013; Krettenauer, 2005). In the present study, findings with respect to the analytical informational style showed a very similar pattern of relationships, such that it positively related to identity commitment, which, in turn, led to lower levels of conflict and increased levels of selfconcept clarity and self-esteem.

On the other hand, the exploratory informational style negatively predicted identity commitment and revealed a positive direct link to ethno-cultural identity conflict. This was unexpected, as the informational style generally has been associated with higher levels of commitment (Bosch \& Card, 2012) and more positive identity outcomes (Berzonsky \& Luyckx, 2008; Crocetti \& Shokri, 2010; Eryigit \& Kerpelman, 2009; Luyckx et al., 2007). Previous studies have shown that, although in the majority of cases the informational style results in an achieved identity with strong commitments, it can also lead to moratorium, which is characterized by high levels of exploration and low levels of commitment (Berman 
et al., 2001; Berzonsky \& Kuk, 2000; Berzonsky \& Neimeyer, 1994). When people are in the process of exploring and revising their identity, it is natural that they are not necessarily ready to commit themselves to specific values and norms; therefore, they may report high levels of exploratory informational style and low levels of commitment. Familiarizing and confronting oneself with sometimes fundamentally different values and beliefs to what is represented by the heritage culture can be an intense experience and prompt self-examination. This might result in a temporary conflict over the identity that can, however, be solved later by moving from exploration to commitment after careful consideration of alternatives and ways in which to resolve conflict. A longitudinal study with immigrants by Szabo et al. (2015) has indeed shown that the exploratory informational style predicts increased use of analytical informational style six months later, which is related to stronger commitment and positive identity outcomes. Seeking information about the values and norms of the receiving society can be an adaptive tool to explore alternative options, which helps immigrants form strong commitments in the long haul.

\section{Normative Styles}

Regarding the two normative orientations, we predicted that the normative style to the host society would increase identity commitment, thus leading to positive identity outcomes, whereas an opposite effect was hypothesized for the normative style to the country of origin, namely that $\mathrm{NCO}$ would be related to decreased levels of identity commitments during cultural transition and more negative identity outcomes. Although a large body of research has shown that those who process on a normative basis are motivated to make commitments (Bosch \& Card, 2012), we expected a negative relationship with NCO, as in the current investigation the measure of identity commitment was highly contextualized reflecting commitments to an immigrant identity and the host society. Therefore, it was predicted that those who expressed a strong normative orientation to the heritage culture would be less willing to commit themselves to the society of residence.

In accordance with our hypothesis, the relationship between normative orientation to the host society and identity outcomes was fully mediated by identity commitment. Recent immigrants, who reported a greater use of NHS, were also more committed to the receiving country, which, in turn, resulted in higher levels of self-esteem and self-concept clarity as well as decreased levels of ethno-cultural identity conflict. Our results indicate that being normative to the host society is associated with a coherent and positively evaluated identity, at least during the first year of cultural transition. This is in line with previous findings in the literature showing that the normative style is often related to an achieved identity (Berzonsky, 
1989, 2008; Berzonsky \& Kuk, 2000; Krettenauer, 2005) and more positive identity outcomes (Beaumont \& Pratt, 2011; Crocetti et al., 2009; Luyckx et al., 2007; Nurmi et al., 1997; Passmore et al., 2005).

In contrast, the relationship between normative orientation to the country of origin and identity outcomes was not mediated by identity commitment. Although the bivariate correlations indicated a significant negative relationship between NCO and identity commitment, this association became non-significant when we controlled for the shared variance between NCO and other identity processing styles, thus the mediational hypothesis was not supported for this style. Instead, we found a moderational effect. More specifically, identity commitment buffered the negative impact of the style on self-esteem. This suggests that being normative to the heritage culture is not necessarily problematic, if acculturating individuals are willing and able to commit themselves to the host society. However, if identity commitment is low, being normative to the heritage culture can have detrimental effects on the evaluation of the self. For a highly normative individual, moving to a new society but not being able to internalize the values and norms represented by the host country and anticipating a meaningful future as an immigrant can cause high levels of stress and anxiety, thus negatively affecting feelings about self-worth and beliefs about the self in general.

It is important to note that previous research findings regarding the relationship between the normative strategy and self-esteem are not completely conclusive. Although most investigations have found positive, weak to medium correlations between the normative style and self-esteem (Crocetti et al., 2009; Eryigit \& Kerpelman, 2009; Johnson \& Nozick, 2011; Luyckx et al., 2007; Passmore et al., 2005), some studies revealed no or, in fact, negative associations between the two constructs (Eryigit, 2010; Miklikowska, 2012). In our study, NCO was negatively related to both self-esteem and self-concept clarity, but identity commitment only buffered the negative effect on self-esteem. Berzonsky (2003) argues that normative commitments are more emotional, compared to informational commitments that are cognitively grounded, which could explain why we found the moderational effect for selfesteem (i.e., affective component) but not for self-concept clarity (i.e., cognitive component). These results show that acculturating individuals who score high on NCO are at risk of developing an unbalanced identity at the early stages of cultural transition; however, identity commitment can successfully attenuate these negative effects. Furthermore, our data suggest that NCO and identity commitment are independent factors of identity development in cultural transition. Findings indicate that it is possible for acculturating individuals to show a 
strong preference to the values and norms of the heritage culture but at the same time develop commitments to the receiving society and the immigrant identity.

\section{Concluding Comments}

In conclusion, the mediational hypothesis was confirmed for the diffuse-avoidant style, the normative style to the host society, the analytical informational style as well as for the exploratory informational style, albeit with an opposite effect to what was predicted.

Furthermore, additional analyses revealed an interaction between identity commitment and the normative style to the country of origin, indicating the buffering effect of commitment. Although commitment functions differently for NCO compared to other identity processing styles during cultural transition, both the moderational and mediational effects point to the essential role of identity commitment in the development of a positive and balanced immigrant identity.

It is, however, important to note that our findings do not provide information about whether our participants developed a new identity or resolved their identity conflicts. We investigated positive and negative identity outcomes to illustrate how social-cognitive identity formation strategies impact the evaluation and perception of the self during the process of identity development. For example, our results indicate that when people undergo an identity crisis in cultural transition, the analytical informational style predicts stronger commitment to the host society, and higher levels of self-esteem and self-concept clarity, and lower levels of perceived conflict over the ethnic and cultural aspects of the identity. This suggests that the analytical informational strategy contributes to a more coherent personal identity, which can potentially buffer the negative effects of acculturative stress and help people reconstruct their social identities as immigrants (Schwartz et al., 2006).

The limitation of this research rests in its cross-sectional design. Identity development occurs over time, and the present study provided only a snapshot of processes involved in identity reconstruction of recent immigrants. Future studies should employ a longitudinal design to investigate the causality of the proposed model and the long-term effects of identity styles. For example, the exploratory informational style was negatively associated with identity commitment in new immigrants; however, as it is suggested by Szabo et al.'s (2015) study, this relationship may change over time and information seeking and exploration can be related to positive identity outcomes in a long-term context. However, it is clearly another research question, for which longitudinal investigations can provide the answer.

It is also worth to note that identity reconstruction occurs in response to a socialcultural environment, therefore other contextual (e.g., immigration policies, cultural 
distance), interpersonal (e.g., perceived discrimination, social support) and intrapersonal variables (e.g., coping styles) can influence the processes examined in the current study. Future studies should explore the antecedents of identity styles and examine potential third variables moderating the relationships among identity styles, identity commitment and identity outcomes during cultural transition.

In spite of the fact that the study used concurrent data, it possesses some clear strengths. Firstly, a specific sample of newly arrived adult immigrants participated in the study, which allowed us to capture identity processes at a very early stage of cultural transition. Previous studies have primarily concentrated on identity development of acculturating adolescents and youth (Phinney, 1990), but significantly less attention has been paid to the intrapersonal processes involved in identity reconstruction of individuals who decided to change cultures as adults.

Secondly, the current research has specifically aimed to explore the dynamic processes contributing to a coherent and positively evaluated immigrant identity. We did not constrain our investigation to specific ethno-cultural identity aspects, i.e., the structural components of identity, but we rather focused on the impact of identity processes on the general evaluation and perception of identity as a whole in new immigrants. This is a novel element in our research, since former studies have aimed attention mainly to ethnic identity formation (Umaña-Taylor, 2001). Newly arrived immigrants confront the acculturative demands of the receiving country that drives them to examine their identity and actively explore identity options in the new country, including but not limited to the ethno-cultural aspects of identity. We argue that, during the early periods of cultural transition, identity exploration is generally focused on identity elements that form the basis of self-definition, such as values and norms.

Thirdly and most importantly, the present research used a developmental approach to investigate the processes underlying identity development and capture the dynamism of identity reconstruction, instead of merely focusing on structural differences in identity. The findings of the study highlight that the integration of developmental theory into research on the identity of acculturating individuals, including both immigrants and ethnic minorities, can fundamentally contribute to the better understanding of intrapersonal mechanisms underpinning identity reconstruction during cultural transition. 


\section{Chapter 4}

Negotiating identity stress during cultural transition: The moderating role of identity styles on the effects of stress appraisal on psychological adjustment over time 


\begin{abstract}
This study investigated the appraisal (threat or challenge) of identity stress in immigrants and its impact on changes in psychological symptoms over time. It also examined how identity styles (informational, normative, and diffuse-avoidant) can moderate these longitudinal relationships. Threat appraisal was expected to facilitate psychological maladjustment, whereas challenge appraisal was predicted to lead to decreased psychological problems over time. In addition, informational styles were hypothesized to enhance, while the diffuseavoidant style was expected to dampen, the positive effects of challenge appraisal on psychological adaptation over time. A six-month longitudinal study with two measuring points was conducted with an international sample of immigrants $(N=137)$. Longitudinal interaction effects between appraisal and identity styles were examined using hierarchical regression analysis. Findings showed that threat appraisal was related to higher levels of psychological symptoms, whereas challenge appraisal was associated with lower levels of psychological problems at both time points. In addition, results revealed that the analytical and exploratory informational styles enhanced the positive effects of challenge appraisal over time. In contrast, styles involving avoidance and normative orientation to one's country of origin dampened the positive effects of challenge appraisal on psychological symptoms six months later. Findings are interpreted within an integrative framework of developmental and acculturation theories.
\end{abstract}




\section{Introduction}

Immigration is on the rise worldwide with more than 230 million people residing outside of their countries of origin (OECD, 2013). Considering the size of the immigrant population, and the increasing cultural diversity in the settlement societies, understanding the acculturation experience of people in cultural transition is likely to be beneficial for individuals and societies. Acculturation refers to the cultural and psychological changes that occur in response to intercultural contact (Berry, 2005). The stress and coping theory provides a theoretical framework, within which the immigrant experience and the changes accompanying cultural transition can be understood, particularly in the prediction of psychological adjustment/well-being (Ward et al., 2001). In the current study, we integrate aspects of developmental and acculturation theory to understand the longitudinal impact of acculturative identity stress on psychological adaptation and to identify strategies fostering positive psychological outcomes in the context of acculturation. More specifically, we examine how threat and challenge appraisals to personal identity during cross-cultural transition influence self-reported psychological problems over time, with particular interest in the moderating role of identity processing styles.

\section{The Transactional Model of Stress and Coping}

According to the transactional model of stress and coping, "psychological stress is a particular relationship between the person and the environment that is appraised by the person as taxing or exceeding his or her resources and endangering his or her wellbeing" (Lazarus \& Folkman, 1984, p. 19). More specifically, when people experience potentially stressful events, they need to make a judgment about the significance of the stressor, evaluate the potential harm it might pose to their psychological functioning (primary appraisal), and assess available resources (secondary appraisal). Depending on whether an event is appraised as an uncontrollable threat versus a controllable challenge, specific coping strategies are mobilized to eliminate the stress-provoking features of the environment.

Not only are appraisals affected by perceptions of controllability of the stressinducing situation, but they also affect emotional reactions to stressors and the coping mechanisms applied to reduce, control or eliminate stress. With respect to threat appraisal, people evaluate the risks and dangers involved in the stressful situation and the potential for loss to be substantially higher than the possibility of successfully coping with the stressor. In contrast, those who appraise a stressor as a challenge tend to interpret the situation as an opportunity to grow and reach mastery; thus, they anticipate more positive future outcomes, 
and most importantly, believe that they have the necessary resources to overcome the stressful experience (Lazarus \& Folkman, 1984).

Threat appraisal has been consistently found to predict negative psychological outcomes in concurrent (Folkman, Lazarus, Gruen, \& Delongis, 1986; Mak, Blewitt, \& Heaven, 2004; Pakenham \& Rinaldis, 2001; Pan, 2011; Skinner \& Brewer, 2002), longitudinal (Florian, Mikulincer, \& Taubman, 1995) and experimental studies (Harvey, Nathens, Bandiera, \& LeBlanc, 2010). Although findings are less conclusive, there is some evidence supporting the positive impact of challenge appraisal on psychological adjustment (Giancola, Grawitch, \& Borchert, 2009; Lazarus \& Folkman, 1984; Mak et al., 2004; Skinner \& Brewer, 2002).

\section{Stress and Coping during Cultural Transition}

Crossing cultures is intrinsically stressful due to the adjustment demands placed on individuals arising from life changes (Holmes \& Rahe, 1967). In addition, many of the changes involved in cultural transition evoke difficulties, including unpleasant experiences due to cultural differences, instrumental and social stressors, and negative emotional factors (Yakhnich, 2008). Specifically, acculturative stress arises when challenges and major life events related to the acculturation experience, including, but not limited to, problems with communication, perceived discrimination or difficulties arising from unfamiliar social norms and rules, become overwhelming and taxing (Berry, 2006). Empirical research demonstrates that the appraisal of these issues as threatening is associated with negative psychological outcomes (Crockett et al., 2007; Lee, Koeske, \& Sales, 2004; Mak \& Neil, 2006; Pan, 2011; Smith \& Khawaja, 2011; Yakhnich \& Ben-Zur, 2008). For example, a study undertaken with Soviet immigrants in Israel found a direct link between threat appraisal of acculturative stressors and increased levels of psychological problems, such as depression and anxiety (Yakhnich \& Ben-Zur, 2008). Similarly, Pan (2011) and Mak and Neil (2006) reported negative associations between threat appraisal and psychological adjustment in international students. In contrast, challenge appraisal has been found to facilitate positive psychological outcomes during the acculturation of international students (Pan, 2008).

Importantly, cultural transition can pose a major threat to immigrants' self-concept and identity integrity. In fact, a large body of research has shown that higher levels of stress accompanying the acculturation experience are associated with lower levels of self-esteem (Abouguendia \& Noels, 2001; Berry \& Sabatier, 2010; Kim \& Omizo, 2005; Navara \& James, 2002; Zamboanga, Schwartz, Jarvis, \& Van Tyne, 2009), sense of coherence (Jibeen $\&$ Khalid, 2010), and self-efficacy (Chen et al., 2008) in immigrant adults and youth. 
Building on the stress and coping framework, Berjot, Girault-Lidvan, and Gillet (2012) found that threat appraisal to personal identity was related to lower levels of selfesteem and increments in stress and suffering. In contrast, those who appraised a situation as a challenge to personal identity reported higher levels of self-esteem and an increased motivation to compete and stand up for their rights. Based on this work, we predicted that threat appraisal to personal identity would be positively related to psychological symptoms (H1). In contrast, challenge appraisal to personal identity was predicted to be negatively related to psychological problems $(\mathrm{H} 2)$.

\section{Negotiating Identity Stress: Identity Styles}

Although cognitive appraisal plays a crucial role in successfully managing difficulties and daily hassles related to the acculturation experience, previous research has revealed that relatively stable individual differences/dispositions can affect psychological outcomes directly (e.g., Ward et al., 2001; Ward, Leong, \& Low, 2004) and moderate the effects of threat and challenge appraisals (van der Zee, van Oudenhoven, \& de Grijs, 2004). For example, an experimental study by van der Zee et al. (2004) indicated that the personality trait of open-mindedness influenced the appraisal of intercultural stressors as threats versus challenges. Specifically, those who scored high on open-mindedness perceived intercultural situations as safer and less threatening. Furthermore, results revealed moderation effects in the stress-adjustment relationship. That is, open-mindedness was related to higher levels of positive affect only under the condition of high intercultural stress.

In developmental psychology, identity styles have been identified as relatively stable dispositions that influence stress management processes and general wellbeing. According to the social-cognitive theory of identity development (Berzonsky, 1989, 2011), people approach identity distress in distinctive ways by using specific information processing and decision making strategies. Berzonsky (1989) distinguished among three social-cognitive identity styles, which refer to specific ways of negotiating identity-relevant issues and coping with identity stress: informational, normative, and diffuse-avoidant.

Those who prefer informational processing tend to evaluate stressors as manageable and solvable; therefore, they apply problem-focused and proactive coping, seek social support, and engage in less wishful thinking and distancing (Beaumont \& Seaton, 2011; Berzonsky, 1992a; Eryigit \& Kerpelman, 2009; Pittman, Kerpelman, Lamke, \& Sollie, 2009; Soenens \& Beaumont, 2008; Soenens et al., 2005). In addition, they react to stressful situations with facilitative anxiety, such that they are motivated to overcome difficulties by trying harder and doing their best (Berzonsky, 1992).The informational style has been 
generally linked to positive psychological outcomes, such as wellbeing (Smits, Soenens, Vansteenkiste, Luyckx, \& Goossens, 2010; Vleioras \& Bosma, 2005), happiness (Beaumont, 2009), and positive affect (Bosch, Segrin, \& Curran, 2012).

The normative style is related to debilitative anxiety (Berzonsky, 1992) and a combination of adaptive and maladaptive coping strategies, such as wishful thinking (Eryigit \& Kerpelman, 2009), distancing (Berzonsky, 1992), and seeking emotional and instrumental social support (Beaumont \& Seaton, 2011; Eryigit \& Kerpelman, 2009). Studies have shown that people with a predominantly normative processing style also use problem-focused and proactive strategies (Beaumont \& Seaton, 2011; Soenens \& Beaumont, 2008; Soenens et al., 2005 ) to a moderate extent. In addition, the style has been associated with lower levels of negative psychological outcomes, including depression (Luyckx et al., 2007; Nurmi et al., 1997), negative affect (Bosch et al., 2012), and psychological symptoms (Faramarzi, Jahanian, Zarbakhsh, Salehi, \& Pasha, 2014).

Those who process on a diffuse and avoidant basis tend to deny problems and try to avoid identity stress. The style has been linked to maladaptive coping strategies, including wishful thinking, distancing, behavioural and mental disengagement, low levels of problemfocused coping and empathy (Beaumont \& Seaton, 2011; Berzonsky, 1992; Pittman et al., 2009; Soenens \& Beaumont, 2008; Soenens et al., 2005). Studies also link the diffuseavoidant style to poorer psychological adjustment in the forms of increased levels of depression (Berzonsky, 2003; Nurmi et al., 1997; Soenens et al., 2011), negative affect (Bosch et al., 2012), psychological symptoms (Faramarzi et al., 2014), and general life distress (Seaton \& Beaumont, 2011) as well as decreased levels of wellbeing (Smits et al., 2010; Vleioras \& Bosma, 2005).

Research has demonstrated that acculturating individuals use similar social-cognitive strategies when dealing with identity stress during cultural transition: analytical and exploratory informational, diffuse-avoidant, normative to the host society, and normative to the country of origin (Szabo, Ward, \& Fletcher, 2015). The exploratory informational style refers to information seeking behaviour and exploration of alternatives through interpersonal contact. In contrast, the analytical informational style captures consideration of alternatives through careful analysis and reasoning. Those who are normative to the country of origin prefer to solve problems and make decisions according to the norms and values represented by their heritage cultures, whereas immigrants with a predominantly normative orientation to the host society tend to automatically internalize the norms and values of the receiving country. Finally, the diffuse-avoidant style involves avoidance of conflicting situations and 
self-relevant feedback during cultural transition. These styles have been associated with aspects of identity in different ways with some styles (e.g., analytical informational and normative to the host society) seeming to be more functional than others (e.g., diffuseavoidant style and normative style to the country of origin) (Szabo \& Ward, 2015).

Studies undertaken with immigrants and members of the mainstream point to the central role of identity styles in the prediction of wellbeing and highlight their differential impact on stress management processes. These findings indicate that social-cognitive information processing styles function as moderators in the stress-adjustment relationship. The moderational hypothesis is further supported by Berzonsky's (1992) theorizing who argued that "the manner in which individuals deal with events or stressors that may invalidate or force revisions in their self-views will vary with their identity style" (pp. 774). Consequently, we propose that stress appraisal to personal identity will interact with identity styles in predicting psychological adjustment over time in acculturating individuals.

More specifically, we hypothesized that the informational styles (exploratory and analytical) would buffer the negative impact of threat appraisal (H3a and H4a) and enhance the positive effects of challenge appraisal ( $\mathrm{H} 3 \mathrm{~b}$ and $\mathrm{H} 4 \mathrm{~b})$ in reducing psychological symptoms over time. The diffuse-avoidant style was predicted to exacerbate the negative impact of threat appraisal (H5a) and to dampen the positive effects of challenge appraisal (H5b) on psychological adjustment over time. Research findings are inconclusive with respect to the normative styles, as associations have been identified with both adaptive and maladaptive stress management and positive and negative identity outcomes (Beaumont $\&$ Seaton, 2011; Berzonsky, 1992; Eryigit, 2010; Eryigit \& Kerpelman, 2009; Szabo \& Ward, 2015). Consequently, we will investigate the interactions between stress appraisals and the normative styles as research questions (RQ1 and RQ2).

\section{Overview}

This study examines the longitudinal influence of threat and challenge appraisal to personal identity on the psychological adjustment of immigrants and the moderating role of identity styles in the stress appraisal-adaptation relationship. In contrast to previous studies, where the focus of research was on general acculturative stress, in the current study we investigate stress management processes related to the identity domain, specifically. In addition, we adopt a longitudinal perspective to assess how stress appraisal and identity processing strategies increase or decrease psychological symptoms of immigrants over time. Although a large body of research has examined stress and coping processes in the context of 
cultural transition, most of these studies employed a cross-sectional design. Relatively little is known about how stress management influences changes in immigrants' wellbeing.

\section{Method}

\section{Design and Sample}

The study employed a two-point longitudinal design with six months in between measuring points. To participate in the study, respondents must have 1) been 18 years of age or older, 2) been living in a country different to their country of origin, and 3) moved to the host society as adults. People living abroad were invited to complete an online survey on immigrant identity, at the end of which respondents could sign up to take part in the second round of data collection. Those who agreed to participate in the longitudinal study were asked to create a unique code by answering four questions (e.g., "What are the first two letters of your mother's first name?", "In which month were you born?"). Next, they were directed to a website unrelated to the survey, where they could provide their email addresses. Six months later participants were contacted with a link to the second online survey. At the beginning of the second survey participants were asked to answer the same four questions. This method allowed us to match survey responses while keeping them anonymous. At the end of both surveys, participants had the chance to go into a lucky draw for Amazon vouchers. Contact information for the draw was collected in a way that did not allow us to match names with survey responses.

At the first measuring point, 280 respondents agreed to take part in the longitudinal study; however, the drop-out rate was $51 \%$ by the second point of measurement. Attrition analysis indicated that the only difference between those who dropped out and those who remained in the study was in immigrant identification; $t(277)=-2.72, p=.007$, Cohen's $d=$ .36. More specifically, participants who completed both surveys reported higher levels of identification with the immigrant category $(M=3.96, S D=2.10$, on a 7-point scale) compared to those who dropped out $(M=3.29, S D=2.02)$.

The final sample included 137 participants (55\% male) who ranged in age from 20 to 65 years $(M=30$ years, $S D=9$ years $)$. Respondents had been living abroad for an average of $M=34$ months $(S D=40.5$ months $)$ at the time of the second round of data collection. The sample included participants from North America (28.5\%), Western Europe (28.5\%), Eastern Europe (12.4\%), South America (10.9\%), Australia and New Zealand (8\%), East-, South-, Southeast Asia and the Pacific (6.6\%), Africa (3.6\%), and the Middle East (1.5\%).

Participants were residing in countries located in Western Europe (41.6\%), East-, South- and Southeast Asia (23\%), North America (16.8\%), Australia and New Zealand (11.7\%), Eastern 
Europe (3.6\%), Africa (1.5\%), and South America (1.5\%). Most participants (82.5\%) were non-native speakers of the official language of the society of residence (average language proficiency: $M=4.12$, and $S D=2.44$ on an 8-point scale). Self-reported English language proficiency (the language of the survey) was high $(M=7.25$ and $S D=.93$ on an 8-point scale). The majority of the sample had previous experience with living abroad (52.6\%) and $38.7 \%$ considered themselves to be visibly different from the majority ethnic group in the receiving country.

\section{Materials}

Identity Style Inventory - Cultural Transition Version (ISI-CT). The ISI-CT is a modified version of the Identity Style Inventory (Berzonsky, 1989). It is specifically designed to assess information processing and decision making strategies underlying identity reconstruction during cultural transition with culturally diverse research participants (Szabo, Ward, \& Fletcher, 2015). The measure includes five scales measuring normative style to the host society (e.g., "I automatically adopt and follow the values of the society in which I live." - NHS, 4 items), normative style to country of origin (e.g., "I automatically adopt and follow the values I was brought up with." - NCO, 5 item), exploratory informational style (e.g., "I've spent a good deal of time reading and talking to others about the values and rules of the country in which I currently live." - EI, 4 items), analytical informational style (e.g., "When facing a decision, I take into account different points of view before making a choice." - AI, 4 items), and diffuse-avoidant style (e.g., "I try not to think about or deal with problems as long as I can." - DA, 5 items).

Participants read the following instruction: "You will find a number of statements about values, attitudes, and ways of dealing with issues as an immigrant in a foreign country. Please, think about your life in the country where you currently live and read each statement carefully." Next, they are asked to indicate on a 5-point scale (anchored by $1=$ not at all like me and $5=$ very much like me) the extent to which they think a statement describes them and their behaviour during cultural transition. The scales have demonstrated acceptable internal consistency, construct, convergent and discriminant validity during the validation process (Szabo et al., 2014). In the present investigation, the reliability of the scales was .78, .70, .58, .70, and .75 for NHS, NCO, EI, AI, and DA, respectively. The exploratory informational style yielded a somewhat lower Cronbach's alpha; however, the item-total correlations were in the expected range $(r=.23-.46)$ and the inter-item correlation was sufficiently high $(r=$ .25) (Briggs \& Cheek, 1986). Since the Cronbach's alpha is a function of the number of item a scale contains, for scales consisting of only a few items, lower alpha values are acceptable 
as long as the item-total correlations and inter-item correlation indicate acceptable internal consistency (Clark \& Watson, 1995; Nunnally, 1978).

Primary Appraisal of Identity Threats (PAIT). The PAIT was developed by Berjot et al. (2012) and consists of four scales assessing the primary appraisal of situations as threats versus challenges to the personal and social identity. Given that the focus of our investigation was on personal identity, we only used the Threat Appraisal to Personal Identity (TPI; 6 items) and Challenge Appraisal to Personal Identity (CPI; 4 items) subscales of the measure. Items were prefaced with the following instruction: "Please, think about the difficulties that you have to face in the country where you currently live. Read each statement carefully and judge the extent to which it describes you and your behaviour during cross-cultural transition". Items were phrased in the context of cultural transition and participants were asked to give responses to statements, such as "When I experience a difficulty during cultural transition, I say to myself that I can't measure up" (TPI) or "When I experience a difficulty during cultural transition, I experience this situation as if I had to take up a challenge" (CPI), on a 5 -point Likert scale (anchored by $1=$ strongly disagree and $5=$ strongly agree). The scales have revealed good internal consistency in previous studies with alphas ranging from .62 to .92 across four studies (Berjot, Altintas, Lesage, \& Grebot, 2013; Berjot et al., 2012). In the present study, both TPI $(\alpha=.82)$ and CPI $(\alpha=.66$; inter-item correlation: $r=.33$; itemtotal correlations: $r=.37-.52$ ) demonstrated acceptable reliability.

Psychological Problems. The measure of psychological problems used in the current study was originally developed for the International Comparative Study of Ethno-cultural Youth (Berry et al., 2006). The scale contains 15 items and participants are asked to rate on a 5 -point scale (anchored by $1=$ never, $5=$ often) how often they experience symptoms like "feeling tired", " feeling sick in the stomach" or "feeling weak all over". The measurement has revealed good reliability in former studies involving participants of mixed cultural backgrounds (Berry et al., 2006), and demonstrated good internal consistency in the present study as well (T1: $\alpha=.92, \mathrm{~T} 2: \alpha=.92)$.

\section{Analytic Plan}

First, preliminary analyses were conducted using bivariate correlations and independent samples t-tests to investigate the relationship between demographic variables (e.g., age, gender, length of stay, language proficiency, ethnic visibility, and previous overseas experience) and the dependent measure. Next, we examined the correlations among the variables of interest, which was followed by testing longitudinal moderation effects. Five sets (one for each style) of three-step hierarchical regression analyses were performed with 
psychological problems at time 2 functioning as the dependent variable. All analyses followed the same procedure. In the first step, we residualized the DV by entering psychological problems at time 1 . This method allowed us to measure change in psychological adjustment over time. Next, we introduced the main effects (i.e., threat and challenge appraisal to personal identity and identity styles). In the last step, interaction terms between appraisal and identity styles were added to the model. Significant interaction effects were imputed into ModGraph (Jose, 2013) and simple slope analyses were employed.

\section{Results}

\section{Preliminary Analysis}

\section{Relationships between demographic variables and the dependent measure.}

Bivariate correlations revealed that psychological problems were unrelated to age, length of stay, English language proficiency, and the proficiency in the official language of the country of residence at both time points. Independent samples t-tests were calculated to examine group differences in psychological symptoms at time 1 and time 2 based on gender, ethnic visibility, and previous sojourner experience. Analyses yielded no significant group differences.

Correlations among the variables of interest. As hypothesized, bivariate correlations indicated that threat appraisal was positively related to psychological problems (see Table 4.1.), whereas challenge appraisal revealed a negative relationship with psychological symptoms at both time points. Therefore, $\mathrm{H} 1$ and $\mathrm{H} 2$ were confirmed. Of the five identity styles, only the diffuse-avoidant strategy showed a significant relationship with psychological maladjustment. More specifically, it was positively related to psychological problems at both time points. In addition, the exploratory informational style yielded a positive correlation with psychological problems at time 2 . The analytical informational and the two normative styles were unrelated to the outcome variable. 
Table 4.1. Correlations and descriptive statistics

\begin{tabular}{|c|c|c|c|c|c|c|c|c|c|c|c|c|}
\hline & 1 & 2 & 3 & 4 & 5 & 6 & 7 & 8 & 9 & $\alpha$ & $M$ & $S D$ \\
\hline 1. Threat Appraisal & 1 & & & & & & & & & .82 & 2.33 & .87 \\
\hline 2. Challenge Appraisal & $-.23^{* *}$ & 1 & & & & & & & & .66 & 3.62 & .72 \\
\hline 3. Exploratory Informational & .14 & $.21^{*}$ & 1 & & & & & & & .58 & 3.76 & .67 \\
\hline 4. Analytical Informational & -.10 & $.24^{* *}$ & $.36^{* *}$ & 1 & & & & & & .70 & 4.34 & .50 \\
\hline 5. Diffuse-avoidant & $.22^{* *}$ & $-.20^{*}$ & $-.22^{* *}$ & $-.26^{* *}$ & 1 & & & & & .75 & 2.65 & .79 \\
\hline 6. Normative to the Host Society & -.10 & .12 & .12 & .03 & .05 & 1 & & & & .78 & 3.18 & .83 \\
\hline 7. Normative to the Country of Origin & $.18^{*}$ & $-.23^{* *}$ & $-.24^{* *}$ & -.10 & $.29^{* *}$ & -.01 & 1 & & & .70 & 2.43 & .70 \\
\hline 8. Psychological Symptoms T1 & $.50^{* *}$ & $-.21^{*}$ & .16 & .00 & $.24^{* *}$ & -.12 & .11 & 1 & & .92 & 2.52 & .77 \\
\hline 9. Psychological Symptoms T2 & $.45^{* *}$ & $-.26^{* *}$ & $.18^{*}$ & -.02 & $.19^{*}$ & -.05 & .10 & $.75^{* *}$ & 1 & .92 & 2.53 & .75 \\
\hline
\end{tabular}

Note. $* \mathrm{p}<.05, * * \mathrm{p}<.01$ 


\section{Regression Analysis}

Results of the regression models are presented in Table 4.2.

Table 4.2. Hierarchical regression of the prediction of change in psychological problems

\begin{tabular}{|c|c|c|c|c|c|c|c|c|c|}
\hline & \multicolumn{3}{|c|}{ Step 1} & \multicolumn{3}{|c|}{ Step 2} & \multicolumn{3}{|c|}{ Step 3} \\
\hline & $\Delta R^{2}$ & $\beta$ & $t$ & $\Delta R^{2}$ & $\beta$ & $t$ & $\Delta R^{2}$ & $\beta$ & $t$ \\
\hline \multicolumn{10}{|l|}{ Exploratory Informational } \\
\hline Psychological Problems T1 & .557 & .75 & $12.84 * *$ & .021 & .67 & $9.94 * *$ & .025 & .68 & $10.24 * *$ \\
\hline Threat Appraisal & & & & & .08 & 1.13 & & .02 & .34 \\
\hline Challenge Appraisal & & & & & -.11 & -1.83 & & -.13 & $-2.06^{*}$ \\
\hline Identity Style & & & & & .08 & 1.34 & & .09 & 1.51 \\
\hline Threat X Style & & & & & & & & .10 & 1.53 \\
\hline Challenge X Style & & & & & & & & -.12 & $-1.99 *$ \\
\hline \multicolumn{10}{|l|}{ Analytical Informational } \\
\hline Psychological Problems T1 & .557 & .75 & $12.84 * *$ & .015 & .68 & $10.10^{* *}$ & .015 & .69 & $10.27 * *$ \\
\hline Threat Appraisal & & & & & .09 & 1.28 & & .07 & 1.00 \\
\hline Challenge Appraisal & & & & & -.09 & -1.51 & & -.09 & -1.44 \\
\hline Identity Style & & & & & .00 & .07 & & .01 & .11 \\
\hline Threat X Style & & & & & & & & -.01 & -.15 \\
\hline Challenge X Style & & & & & & & & -.12 & $-2.12 *$ \\
\hline \multicolumn{10}{|l|}{ Diffuse-avoidant } \\
\hline Psychological Problems T1 & .557 & .75 & $12.84 * *$ & .016 & .69 & $10.09^{* *}$ & .030 & .70 & $10.49^{* *}$ \\
\hline Threat Appraisal & & & & & .09 & 1.31 & & .10 & 1.45 \\
\hline Challenge Appraisal & & & & & -.09 & -1.56 & & -.11 & -1.86 \\
\hline Identity Style & & & & & -.02 & -.33 & & -.01 & -.20 \\
\hline Threat X Style & & & & & & & & .12 & 1.86 \\
\hline Challenge X Style & & & & & & & & .19 & $3.02 * *$ \\
\hline \multicolumn{10}{|l|}{ NCO } \\
\hline Psychological Problems T1 & .557 & .75 & $12.84 * *$ & .016 & .68 & $10.15^{* *}$ & .016 & .69 & $10.22 * *$ \\
\hline Threat Appraisal & & & & & .09 & 1.31 & & .10 & 1.41 \\
\hline Challenge Appraisal & & & & & -.09 & -1.56 & & -.10 & -1.73 \\
\hline Identity Style & & & & & -.02 & -.30 & & -.02 & -.42 \\
\hline Threat X Style & & & & & & & & .02 & .35 \\
\hline Challenge X Style & & & & & & & & .13 & $2.11 *$ \\
\hline \multicolumn{10}{|l|}{ NHS } \\
\hline Psychological Problems T1 & .557 & .75 & $12.84 * *$ & .019 & .69 & $10.24 * *$ & .003 & 0.69 & $10.19^{* *}$ \\
\hline Threat Appraisal & & & & & .09 & 1.32 & & 0.09 & 1.28 \\
\hline Challenge Appraisal & & & & & -.10 & -1.61 & & -0.09 & -1.55 \\
\hline Identity Style & & & & & .06 & 1.09 & & 0.07 & 1.12 \\
\hline Threat X Style & & & & & & & & -0.04 & -0.60 \\
\hline Challenge X Style & & & & & & & & -0.05 & -0.78 \\
\hline
\end{tabular}

Note. ${ }^{* *} \mathrm{p}<.01,{ }^{*} \mathrm{p}<.05$; Dependent measure: Psychological Problems T2; NCO, Normative Style to the Country of Origin; NHS, Normative Style to the Host Society 
Exploratory Informational Style. The three-step regression model explained $60.4 \%$ of variance in psychological problems at time $2 ; F(6,126)=31.96, p<.001$. In the final step, challenge appraisal had a significant main effect $(\beta=-.13, p=.048)$, which was qualified by a significant challenge appraisal x exploratory informational style interaction $(\beta$ $=-.12, p=.049)$. In line with our hypothesis $(\mathrm{H} 3 \mathrm{~b})$, simple slope analyses indicated that the exploratory informational strategy enhanced the positive effects of challenge appraisal on psychological adjustment (see Figure 4.1). More specifically, challenge appraisal predicted a decrease in psychological problems over time under medium $(t(130)=-2.05, p=.042)$ and high $(t(130)=-2.56, p=.012)$ levels of the exploratory informational style.

Analytical Informational Style. The main effects and interactions explained 58.7\% of variance in psychological problems at time $2 ; F(6,126)=29.90, p<.001$. In the final step, none of the variables showed a significant main effect; however, challenge appraisal and the analytical informational style interacted in predicting changes in psychological problems over time $(\beta=-.12, p=.036$; Figure 4.2). Post hoc analyses revealed that challenge appraisal led to decrements in psychological symptoms over time when participants reported increased uses of the analytical informational style $(t(130)=-2.57, p=.011)$. Under low and medium levels of the analytical style challenge appraisal was unrelated to changes in psychological problems. Consequently, H4b was confirmed.

Diffuse-avoidant Style. The three-step model was significant and explained a total of $60.3 \%$ variance in psychological problems at time $2 ; F(6,126)=31.93, p<.001$. There were no significant main effects, but challenge appraisal yielded a significant interaction with the diffuse-avoidant style $(\beta=.19, p=.003)$. As depicted by Figure 4.3 , the diffuse-avoidant style dampened the positive effects of challenge appraisal over time, which supported H5b. In particular, challenge appraisal predicted decreased psychological symptoms over time only when diffuse-avoidance was low $(t(130)=-3.23, p=.001)$.

Normative Style to the Country or Origin (NCO). The regression model was significant and explained $58.8 \%$ of variance in the outcome variable; $F(6,126)=30.02, p<$ .001 . In the third step, only the challenge appraisal x NCO interaction was significant $(\beta=$ $.13, p=.037)$. Subsequent simple slope analyses indicated that challenge appraisal was associated with decreased psychological problems over time under low levels of $\mathrm{NCO}(t$ $(130)=-3.64, p=.009$, see Figure 4.4.) indicating that NCO dampened the positive effects of challenge appraisal.

Normative Style to the Host Society. The three-step regression model yielded neither significant main effects, nor significant interactions. 
Figure 4.1. The exploratory informational style at time 1 enhanced the positive effects of challenge appraisal at time 1 on psychological adjustment over time.

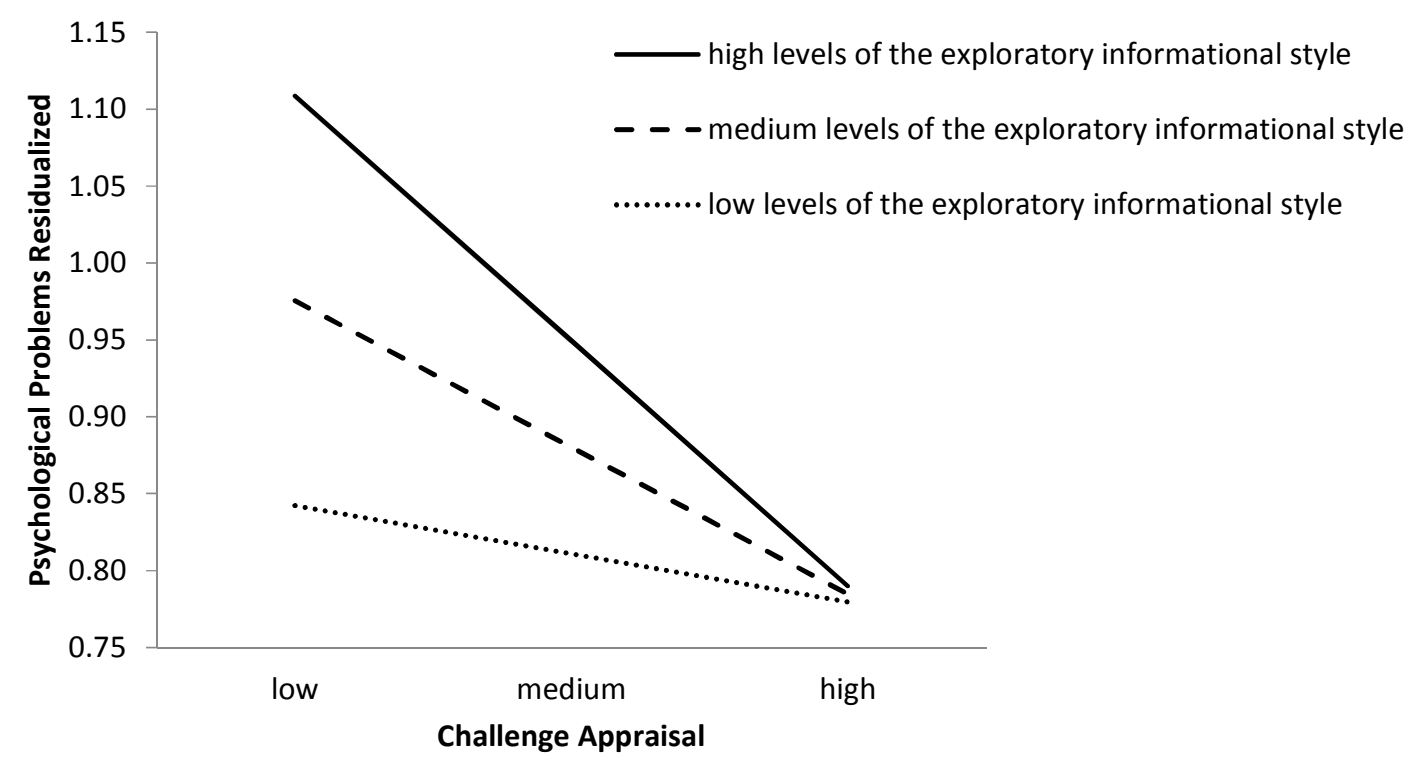

Note. Simple slope analysis for low levels of the exploratory informational style; $t(130)=-.63, n s$, for medium levels of the exploratory informational style; $t(130)=-2.05, p=.042$, and for high levels of the exploratory informational style; $t(130)=$ $-2.56, p=.012$.

Figure 4.2. The analytical informational style at time 1 enhanced the positive effects of challenge appraisal at time 1 on psychological adjustment over time.

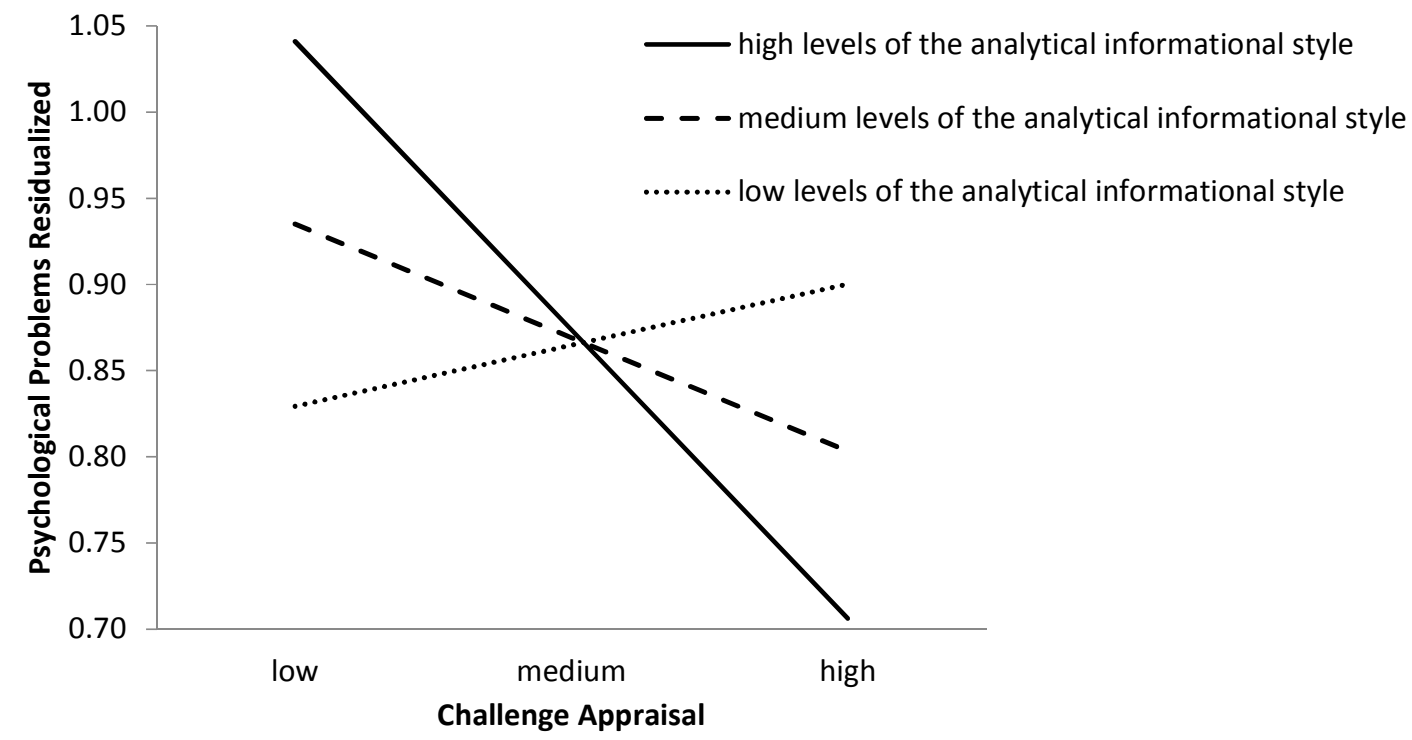

Note. Simple slope analysis for low levels of the analytical informational style; $t(130)=.53, n s$, for medium levels of the analytical informational style; $t(130)=-1.45, n s$, and for high levels of the analytical informational style; $t(130)=-2.57, p=$ .011 . 
Figure 4.3. The diffuse-avoidant style at time 1 dampened the positive effects of challenge appraisal at time 1 on psychological adjustment over time.

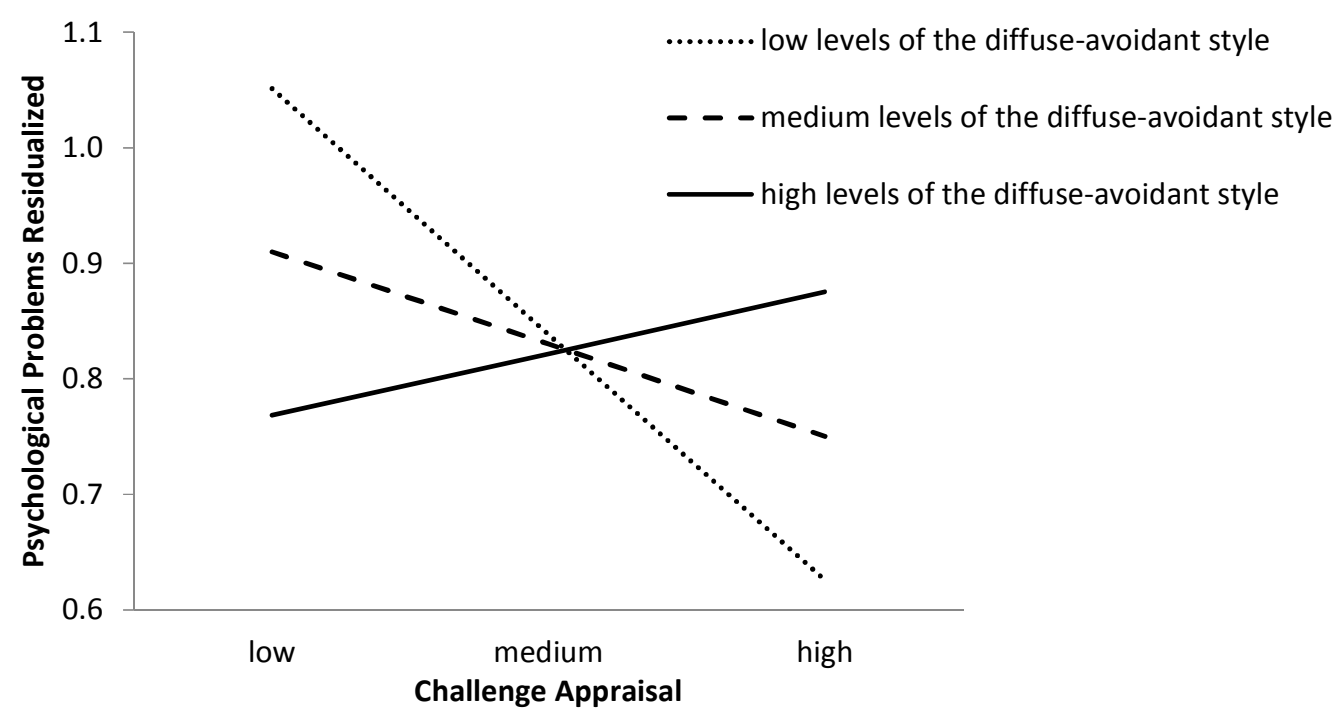

Note. Simple slope analysis for low levels of the diffuse-avoidant style; $t(130)=-3.23, p=.001$, for medium levels of the diffuse-avoidant style; $t(130)=-1.81, p=.072$, and for high levels of the diffuse-avoidant style; $t(130)=.89, n s$.

Figure 4.4. The normative style to the country of origin at time 1 dampened the positive effects of challenge appraisal at time 1 on psychological adjustment over time.

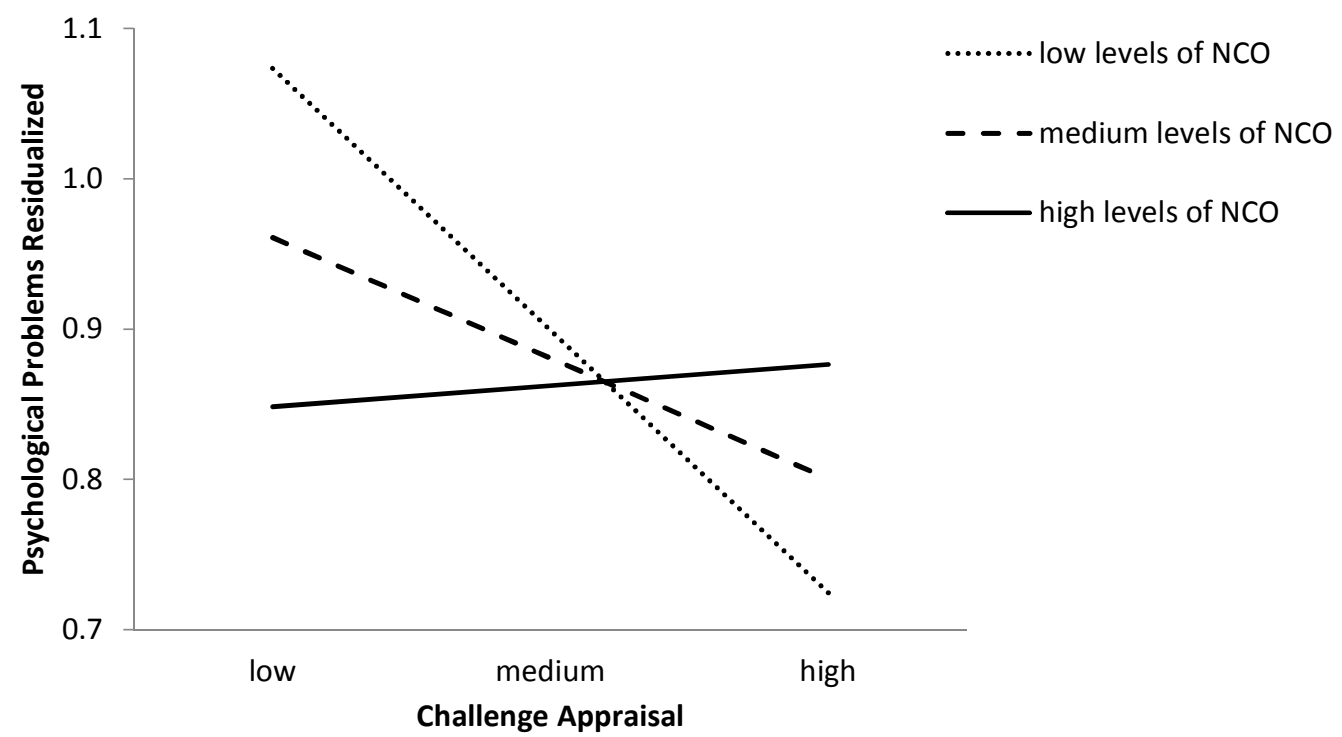

Note. Simple slope analysis for low levels of the normative style to the country of origin; $t(130)=-3.64, p=.009$, for medium levels of the normative style to the country of origin style; $t(130)=-1.77, p=.078$, and for high levels of the normative style to the country of origin style; $t(130)=.23, n s$. 


\section{Discussion}

The current study investigated the longitudinal impact of identity stress appraisals and strategic identity processing styles on the psychological adjustment of people in cultural transition. In particular, we tested interactions between appraisal and identity strategies in predicting changes in psychological symptoms over time. First, bivariate relationships were examined between appraisal and psychological outcomes. In line with our hypotheses, threat appraisal was associated with increased levels of psychological symptoms, whereas challenge appraisal yielded negative relationships with psychological problems. These results support previous studies linking threat appraisal to negative, and challenge appraisal to positive, psychological outcomes (Berjot et al., 2012; Folkman et al., 1986; Giancola et al., 2009; Lazarus \& Folkman, 1984; Mak \& Neil, 2006; Pakenham \& Rinaldis, 2001; Pan, 2008, 2011; Skinner \& Brewer, 2002).

Next, the longitudinal effects of appraisals on psychological problems were tested. No significant main effects were found for threat appraisal. Threat appraisal was initially associated with higher levels of symptoms, and this association remained stable over time. This finding is in line with a previous study by Florian et al. (1995) who investigated the relationship between appraisal and psychological outcomes using both cross-sectional and longitudinal designs. They found that threat appraisal was related to lower levels of wellbeing and increased levels of psychological distress when measured concurrently. Threat appraisal also predicted decrements in wellbeing over time; however, it had no longitudinal effects on changes in psychological symptoms. Although threat appraisal did not increase problems over time, perceiving obstacles during cultural transition as threats to one's personal identity was associated with higher levels of psychological difficulties at both time points suggesting that threat appraisal contributed to sustained levels of stress in acculturating individuals.

In contrast, the ability to appraise stressors accompanying cultural transition as challenges, as opposed to threats, was linked to lower levels of initial problems, and most importantly, had the capacity to reduce symptoms over time, albeit this effect was found only in one of the regression models. This provides supporting evidence for Lazarus and Folkman's (1984) contention that challenge appraisal plays a crucial role in successful adaptation. Only a few studies have been previously undertaken to investigate the longitudinal effects of challenge appraisal on psychological outcomes, and results are not conclusive. For example, in Florian et al.'s (1995) investigation challenge appraisal had no significant main effects on either wellbeing or psychological distress over time. However, in another study undertaken by Skinner and Brewer (2002) to examine temporal patterns of 
stress appraisals during performance tasks, challenge appraisal was found to predict more confident coping expectancies, increments in positive emotions, and better performance. Our findings indicate that challenge appraisal can be functional during acculturation to facilitate positive adjustment; however, as only one of the regression models yielded a significant longitudinal effect, this needs to be replicated by future studies.

In addition to its main effect, challenge appraisal interacted with four (out of five) identity styles investigated in the present research. In line with our hypotheses, increased uses of the exploratory and analytical informational strategies enhanced the positive effects of challenge appraisal in reducing psychological symptoms over time. Those who show a preference for the exploratory strategy are motivated to seek information through interpersonal contact in order to make sense out of issues that arise during stressful times in cultural transition. The analytical style is also related to increased needs for information and understanding of the stress-inducing factors; however, people who score high on this strategy tend to use more inward directed information processing, such as careful consideration of alternatives and thinking thoroughly about options, as opposed to asking help from others (Szabo et al., 2015).

The separation of the two styles is specific to the cross-cultural context, as in the general population they form one general strategy of informational processing. Previous studies undertaken with immigrants have shown that the two styles are moderately correlated and that the exploratory often precedes the analytical one (Szabo \& Ward, in press; Szabo et al., 2014). Our findings indicate that challenge appraisal in conjunction with informational strategies, whether they are manifested in interpersonal information seeking or intrapersonal information processing, are adaptive in reducing psychological symptoms in the context of cross-cultural transition.

With respect to the diffuse-avoidant style and the normative style to the country of origin, analyses indicated a dampening effect, such that increased uses of these strategies attenuated the positive impact of challenge appraisal on psychological problems over time. Those with a predominantly diffuse-avoidant style are inclined to ignore and avoid dealing with identity distressing situations; therefore, they try to delay taking actions and tend to deny that problems exist (Berzonsky \& Ferrari, 2009). In contrast, the normative style to the country of origin involves high levels of conformity to one's heritage culture; thus, people characterised by this style have a tendency to negotiate identity issues according to the rules, norms, and values with which they were brought up, even if they are not necessarily adaptive in the society of residence (Szabo et al., 2015). 
One thing the two strategies have in common is that they involve avoidance of the stressor and withdrawal of one's resources. However, challenge appraisal requires approach behaviours, as obstacles cannot be normally overcome unless they are acknowledged and addressed. Consequently, our findings indicate that perceiving a stressful situation as a challenge is unsuccessful at diminishing psychological problems over time, if people mobilize inappropriate strategies to approach and address the stressor. These interaction effects highlight the importance of the compatibility between appraisal and the strategies used to address the stressor, and thus provide further support for the goodness of fit hypothesis (Forsythe \& Compas, 1987).

The goodness of fit hypothesis postulates that the effectiveness of stress management depends on the compatibility between stress appraisal and the strategies applied in response to the stressor. For example, it is suggested that secondary control strategies (e.g., acceptance and positive reinterpretation) are effective at buffering the negative effects of stress when stressors are appraised as uncontrollable (Compas, Connor-Smith, Saltzman, Thomsen, \& Wadsworth, 2001; Connor-Smith \& Compas, 2004; Szabo, Ward, \& Jose, in press; Thurber $\&$ Weisz, 1997). By contrast, problem-focused and active strategies are more adaptive when the stressful experience is appraised as controllable (Folkman \& Lazarus, 1980; Forsythe \& Compas, 1987; Park, Armeli, \& Tennen, 2004). Similarly, our findings indicate that perceiving acculturative stressors as controllable challenges lead to positive psychological outcomes when combined with active, informational identity strategies. However, when passive and avoidant strategies are in use, challenge appraisal per se does not have the capacity to reduce symptoms and promote adjustment. In short, challenge appraisal tends to be adaptive and functional, but its effects are moderated by control responses.

No significant results were found in relation to the normative style to the host society indicating that the strategy has neither positive nor negative effects on short-term psychological adjustment. This strategy can help people become socially successful through the automatic internalization of the norms and values represented by the receiving culture. Although this style can have positive implications in terms of commitment and self-esteem (Szabo \& Ward, 2015), automatically adopting the value- and norm system of the society of settlement may involve the rejection of certain features of the heritage culture, which can have negative effects in terms of mental health and psychological adjustment (Ward \& Kennedy, 1994; Ward \& Rana-Deuba, 1999). We propose that a third variable, such as cultural similarity, might also influence the interaction between appraisal and the normative style to the host society. For immigrants whose heritage culture is highly similar to the culture 
of the receiving country, following the rules represented by the locals can be an obvious choice, providing an easy, effortless and smooth transition. However, for those coming from a culture that is greatly different from the host cultural environment, being normative to the settlement society can involve a number of difficulties socially and psychologically. Consequently, automatically conforming to the host norms might have both positive and negative influences, which cancel each other out in the current study employing a culturally diverse sample.

\section{Limitations, Strengths, and Conclusions}

In the present investigation, psychological adjustment was exclusively assessed in the form of negative outcomes. Our findings indicate that challenge appraisal is effective at reducing psychological symptoms over time, especially when combined with appropriate identity processing strategies. However, it remains unclear whether it is a protective factor only or if it can also facilitate positive adjustment, such as increased levels of wellbeing and life satisfaction. This ambiguity might also account for the lack of results with threat appraisal to personal identity, as it has been shown that threat appraisal predicts decrements in wellbeing, but not increments in psychological symptoms, over time (Florian et al., 1995).

In addition, we evaluated the impact of identity stress appraisal and identity processing styles on psychological adjustment. Crucially, cross-cultural adaptation has both psychological and sociocultural indicators (Searle \& Ward, 1990; Ward et al., 2001). Studies have shown that sociocultural adjustment is affected by acculturative stress (Ataca \& Berry, 2002; Sochos \& Diniz, 2012; Yijala, Lonnqvist, Jasinskaja-Lahti, \& Verkasalo, 2012) -- it is fair to assume that stress appraisal and identity processing styles could also play an important role in the long term sociocultural adaptation of immigrants. These research questions should be tested in future investigations.

Notable strengths of the present inquiry include the longitudinal design, which allowed us to investigate change in psychological symptoms over a six months period, and the diverse sample of international immigrants participating in the study. In addition, instead of measuring acculturative stress, we assessed stress appraisal to personal identity, i.e., the perceived controllability and significance of the stressor. Cultural transition is intrinsically stressful; however, there are substantial individual differences in the intensity and kinds of reactions to the difficulties involved in the acculturation process. The evaluation of a stressor as challenging vs. threatening or controllable vs. uncontrollable determines not only emotional and behavioural responses, but ultimately may influence the outcome of the stressadjustment relationship. 
We focused on a specific aspect of acculturative stress, namely stressors affecting the identity domain. Previous studies have mainly concentrated on how interpersonal stressors, such as discrimination and communication difficulties, influence psychological outcomes during transition. Identity stress and its long term impact on cross-cultural adjustment have received considerably less attention in acculturation research, even though a substantial body of literature has highlighted the role of a positive, integrated and balanced identity in the cross-cultural adaptation of immigrants (Aycan \& Berry, 1996; Costigan et al., 2010; Jasinskaja-Lahti \& Liebkind, 2001; H. H. Nguyen et al., 1999; Safdar et al., 2006; Usborne \& Taylor, 2010; Ying et al., 2007).

The present study also broadens our understanding of the functionality of identity styles in general. On one hand, our findings draw attention to identity stress as a form of acculturative difficulties and point to the key role of appraisal in the stress management and adjustment process. On the other hand, our results emphasize the importance of identity processing styles and their functionality in facilitating, or interfering with, the stressadjustment process. Previous investigations have mainly focused on how identity styles promote specific coping mechanisms (Berzonsky, 1992); however, the interaction effects indicate that social-cognitive identity styles can be adopted as control strategies when confronting identity stress.

These findings also have applications for health care professionals, counsellors and trainers working with immigrant clients. Cognitive reframing of stressors as challenges, instead of threats, greatly impacts the psychological wellbeing of immigrants. However, identity processes can facilitate, or interfere with, the stress management process, such that the positive effects of challenge appraisal can be enhanced or diminished depending on the identity styles utilized in response to acculturative stress. Thus, stress management trainings and workshops specifically designed to promote the cross-cultural adaptation of acculturating individuals could greatly benefit from placing emphasis on coping with acculturative stressors specifically linked to identity.

In conclusion, these findings provide novel information about the longitudinal impact of identity stress appraisals on the psychological adjustment of acculturating individuals, and point to the significance of challenge appraisal in adaptation. Furthermore, our results suggest that it is not enough to frame difficulties simply in positive and favourable terms; it is also important for identity distressing situations to be approached with effective and appropriate social-cognitive identity styles. The present investigation highlights the need for more process-oriented approaches in the study of acculturation. We believe that integrating 
approaches for developmental and cross-cultural psychology to investigate identity stress management in cultural transition can give deeper insights into the mechanisms underlying the cross-cultural adaptation of immigrants, sojourners, and ethnic minorities. 


\section{Chapter 5}

General discussion 


\section{General Discussion}

The present research programme set out to provide an in-depth empirical examination of three key aspects of early acculturation. In particular, the series of studies reported in chapters 2, 3, and 4 aimed to: 1) identify the range of social-cognitive identity processing styles applied in response to an acculturation identity crisis; 2) determine positive and negative pathways to the development of a committed and consolidated immigrant identity; and 3) understand the role of identity styles in acculturative identity stress management and the prediction of immigrant wellbeing over time.

In the current thesis, each chapter was presented with its own discussion, which included a general summary of main findings; consideration of the limitations and strengths of the research conducted; implications of findings for theory, research, and application as well as general conclusions. Therefore, instead of giving a detailed and exhaustive rehash of the main outcomes of each study, the goal of the general discussion is to review the underlying theoretical assumptions of the research programme, outline its conceptual and empirical contributions to acculturation research, delimit the boundaries of my research project, define implications for the immigrant population and the host society, and formulate recommendations for future research.

\section{Theoretical Underpinnings and Key Findings}

\section{Examining Acculturation and Identity Through the Lens of Developmental Theory}

This research programme was undertaken as a response to a growing need for a more process-oriented approach to acculturation and identity. After reviewing the acculturation literature, I came to the realization that, although mechanisms and strategies underlying identity development in cultural transition and acculturation have been theorized (e.g., Schwartz et al., 2006), they mostly remained as plausible hypotheses lacking empirical testing. In the field of developmental psychology, however, studying processes involved in identity development has formed the scope of investigations for decades.

Drawing on the psychosocial theory of identity development (Erikson, 1968), two theoretical assumptions served as the main pillars of the research series reported in this thesis. According to the first assumption, the development and reconstruction of identity is fundamentally influenced, and to some extent constrained, by the wider socio-cultural context, in which the individual is embedded (Bosma \& Kunnen, 2001). Changes in the socio-cultural context function as triggers for identity reconstruction, hence acculturating individuals - immigrants, in particular - form a unique population for the study of situationinduced identity crises. Secondly, it is argued that identity development is a life-long project 
that continues beyond adolescence. Changes in aspects of identity and the examination of identity-relevant issues in adult immigrants post transition should serve as an important focus of research on acculturation and identity. I believe that in order to understand the impact of cultural transition on the identity of adult immigrants, developmental theory needs to be integrated into acculturation research.

As a first step to achieve this goal, Berzonsky's (1989) social-cognitive theory of identity styles has been adapted to the immigrant population in order to investigate the strategies underlying the development of a committed immigrant identity. One of the main outcomes of my effort to incorporate elements of developmental theory in to the study of acculturation was the construction and validation of a new assessment tool that is capable of capturing the social-cognitive processes underpinning exploration during an acculturation identity crisis. Furthermore, Berzonsky's framework has been extended to the study of acculturation and identity by testing a model of identity development with recent immigrants. Identifying both positive and negative pathways to identity outcomes (in the forms of selfesteem, self-concept clarity, and ethno-cultural identity conflict), the research provided evidence for identity processing styles as driving mechanisms in the identity reconstruction of new immigrants and shed light on the central role of developing commitments to an immigrant identity.

\section{Examining Acculturation and Identity Over Time}

Another reason why the process-oriented approach has been relatively neglected in the acculturation arena is that it requires longitudinal research. Notwithstanding that conducting studies with multiple measuring points is expensive and time consuming, longitudinal designs give us the opportunity to identify trends across a period of time and observe changes in a more accurate way. Furthermore, repeated observations of the same individuals allow researchers to make conclusions about the temporal order of events. In the context of this research programme, conducting a longitudinal study as an addition to the larger crosssectional investigations was crucial in order to examine the stability of identity processing styles and to uncover predictors of immigrant wellbeing over time.

The longitudinal investigation served two important objectives. First, it aimed to examine variability or change in the utilization of specific social-cognitive identity processing styles over a six month period. The findings supported theoretical assumptions in that individuals demonstrated relatively stable preferences in the use of identity strategies; however, the research also elucidated the relationship between exploratory and analytical informational styles by indicating the temporal primacy of the former. This finding allowed 
us to gain a more accurate understanding of why the two types of informational processing emerged as separate strategies in the context of cultural transition. The second goal was to gain a clearer perspective on the role identity styles play in the prediction of psychological adjustment. Examining identity styles in connection with acculturative identity stress management uncovered new ways of enhancing immigrant wellbeing over time.

\section{Conceptual and Empirical Contributions}

Applying elements of developmental theory within the existing frameworks of acculturation opened up a new perspective on the study of identity in acculturating individuals. Each empirical paper presented in this thesis has the potential to make important conceptual and empirical contributions to both areas of psychology. First, I discuss the implications of findings for the field of acculturation with a particular emphasis on the benefits of the developmental approach. The following section focuses on the other side of the coin by drawing attention to how acculturation research can enhance developmental psychology in the understanding of human development in cultural context.

\section{What Can Acculturation Research Offer to Developmental Theory?}

In the globalizing world where intercultural contact has become an important facet of daily life in most developed countries, acculturation should be considered as a significant factor in human development. Ethnic identity development has received substantial attention in developmental psychology; however, these investigations focused mainly on adolescents and young adults. Relatively little research has been concerned with identity processes in first generation immigrants and individuals who decided to change cultures as adults.

Furthermore, acculturation is a bidirectional process that affects not just immigrants and members of ethnic minorities, but also involves psychological and socio-cultural changes for host nationals. In short, in order to gain a deeper understanding of identity formation in context, developmental theory and research should address issues of social change in the global era.

The findings of the current thesis make contributions to developmental theory in at least two important ways. On one hand, results provide a significant empirical contribution to the social-cognitive theory of identity styles. Although studies have been undertaken with immigrant and ethnic minority youth, these investigations examine the role of identity processing strategies in normative identity crises evoked by developmental changes (Schwartz et al., 2005; Schwartz \& Montgomery, 2002). This is the first research programme that examines identity processing styles in connection with acculturation identity crises in samples of adult immigrants. 
Identity processing strategies unique to the acculturating population have been uncovered. The research suggests that the normative style can play out in two different ways in a cross-cultural context; normative orientation to the host society and normative orientation to the country of origin. In addition, the exploratory and analytical informational styles emerged as independent dimensions and showed temporal separation as well. It is also important to highlight that the two informational styles predicted identity outcomes in opposite ways. These are novel findings providing important information for the evolving line of research on the components of exploration behaviour. Syed and colleagues (2013) have indicated that ethnic identity search is related to increased levels of identity confusion, whereas ethnic identity participation is linked to positive outcomes. Similarly, the results of the present research suggest that the exploratory informational style is associated with negative identity outcomes, at least in a short-term context.

On a more conceptual level, two main points need to be emphasized. The studies presented in this thesis included samples of adult immigrants with a wide age range from 18 to 78 years old. This indicates that identity development is likely to be a life-long project that continues beyond adolescence and emerging adulthood. Although in the current research the moderating role of age was not investigated, it is fair to assume that one's age at the time of cultural transition might influence the overall process and the outcome of immigrant identity reconstruction.

The research programme also highlights that situational and contextual changes often serve as triggers for identity reconstruction. As the pace of migration increases, acculturation identity crises will become a common experience for most acculturating individuals. It should be an urgent issue for developmental psychology to respond to this changing phenomenon by generating theories that are inclusive of adult immigrant populations and by conducting empirical research in order to assist professionals working with acculturating individuals. This project can form a launching pad for a new line of research on situation-induced identity crises and the processes involved in immigrant identity development.

\section{The Benefits of Applying Developmental Theory in the Context of Acculturation Research}

Adopting theories from other areas of psychology to explain social and psychological changes involved in cultural transition is not new in acculturation research. For example, the ABCs of acculturation (Ward et al., 2001) provides an integrative framework of stress and coping (Lazarus \& Folkman, 1984), culture learning theory (Argyle, 1967), social identity theory (Tajfel, 1978; Turner, Hogg, Oakes, Reicher, \& Wetherell, 1987), and integrative 
threat theory (Stephan, Ybarra, \& Bachman, 1999) to explain the affective, behavioural and cognitive aspects of cross-cultural adaptation. However, it has been relatively neglected how developmental mechanisms influence the immigrant experience and interplay with the affective, behavioural and cognitive processes of acculturation. This has resulted in a paucity of longitudinal investigations and research focusing on intrapersonal strategies and mechanisms underlying psychological change, such as identity development, during cultural transition.

The fact that identity theory has been researched for many decades by developmental psychologists provides an unparalleled opportunity for acculturation researchers to use already existing and widely validated assessment tools to study identity in a cross-cultural context. Of course, these measures often need to be modified and adapted for a certain population; however, they can function as important starting points for the development of scales specifically designed for assessing identity processes in acculturating individuals.

The lack of valid and reliable measurements is often a reason why certain fields do not progress in a timely fashion. Without having the necessary tools to assess processes underlying identity development during acculturation, it will remain an under-researched area of inquiry. Chapter 2 presented a series of studies that was undertaken to adapt a measure of identity strategies to assess processes involved in identity exploration during cultural transition. The development of the Identity Style Inventory - Cultural Transition Version was the first step to supplement the toolboxes of acculturation researchers and enable the study of identity processing styles in the context of cultural transition.

Identity outcomes and structural differences in identity have been studied in the fields of acculturation and cross-cultural psychology (see Benet-Martinez \& Haritatos, 2005; Ward et al., 2011). These investigations not only offered valid measures for the assessment of identity structure and outcomes, but also made valuable conceptual contributions to identity theory in a cross-cultural context. At the same time, however, it remains unclear what factors account for individual differences in identity structures and outcomes. Why is it that some people achieve a positive identity during cultural transition, whereas others with similar demographic backgrounds are characterized by poor outcomes? The second paper, presented in Chapter 3, provided empirical evidence for the utility of the developmental approach in predicting identity outcomes among newly arrived adult immigrants. The findings highlight how a developmental perspective can help us gain insights into the mechanisms underpinning individual differences in identity structure and outcomes. 
Finally, the longitudinal study on acculturative identity stress management provides a successful example of incorporating developmental theory into an existing framework of acculturation research. The ABC model (Ward, 2001) suggests stress and coping as a useful framework for investigating the affective components of cross-cultural adjustment. Drawing on Lazarus and Folkman's (1984) paradigm of stress and coping, we examined the capability and efficiency of identity processing styles to buffer the negative effects of acculturative identity stress and to predict positive psychological adjustment over time. In conclusion, the results of the studies highlight that developmental theories are compatible with acculturation frameworks and have the potential to broaden the horizons of research with acculturating individuals and groups.

\section{Immigrant Identity: An Alternative Framework}

The present thesis introduces the concept of immigrant identity as an alternative framework for the study of identity development in the context of cultural transition with mobile acculturating individuals. This is a novel perspective on acculturation and identity that allows the study of identity exploration and commitment with a focus on personal identity aspects. In addition, it allows the study of acculturating groups for whom the reconciliation of ethnic and national identity aspects is not of primary concern (e.g., newly arrived or recent immigrants and economic migrants).

The immigrant identity is a special case of identification, as it is automatically assigned and initially based on a legal category, but can evolve into a strong and influential social identity. This is corroborated by the fact that a reasonable proportion of participants were permanent residents, or even citizens, in the society of settlement, but still self-identified as immigrants and willingly took part in a study on immigrant identity.

Many researchers have argued that social and cultural changes can give rise to identity crises (see Baumeister et al., 1985 or Erikson, 1968). However, I argue that moving to a new cultural system alone is not necessarily a trigger for acculturation identity crises. What makes identity-relevant issues particularly salient for immigrants is cultural transition combined with fundamental changes in one's legal status, social mobility, and group memberships. Furthermore, it is important to emphasize that the immigrant identity is created through interactions with members of the dominant group. Host nationals' perceptions of and attitudes toward diversity and immigration determine how they treat, communicate with, and approach newcomers. Ultimately, intergroup relations have a substantial influence on how and by what means immigrants engage in identity reconstruction and develop new commitments in the context of the settlement society. 


\section{Minding the Gap: The Limits of the Contribution}

The current research programme possesses limitations on two counts. A developmental perspective was adopted to identify the mechanisms underlying the identity development of immigrants. Although both longitudinal and concurrent data were collected to answer the research questions, in Chapter 3 the proposed model of immigrant identity development was tested with a cross-sectional sample, thus providing only a snap-shot of the dynamic interplay among identity processes, commitment, and identity outcomes. The longitudinal investigation indicated significant findings with respect to the temporal stability of identity processing styles and the utility of these strategies in managing acculturative identity stress. However, the longitudinal design used in the current research included only two measuring points, so that conclusions could not be made regarding developmental trends.

On the second account, it is important to highlight that the diversity of the samples is both a strength and a limitation. On one hand, inviting participants with diverse cultural backgrounds, as opposed to focusing on specific migrant groups or receiving societies, allowed us to give a more realistic representation of the immigrant population and test predictions that potentially generalize across ethnic and cultural groups. On the other hand, this diversity, constrained by the overall sample size did not permit meaningful cultural group comparisons or equivalence testing across host societies and countries of origin. Further, investigating cultural differences (i.e., perceived cultural distance) and the impact of wider contextual socio-cultural factors (i.e., immigration policies or population diversity) could make valuable contributions that strengthen the theoretical underpinnings of research on acculturation and identity.

\section{Three Avenues for Future Research}

Based on the data in hand, I foresee three distinct paths through which the body of knowledge on acculturation and immigrant identity could be advanced through future research. First, it is necessary to broaden the range of designs and methods used to investigate identity development in acculturating individuals. In order to capture trends of identity development and change over time, more long-term longitudinal research projects are needed. In addition, the field of acculturation could greatly benefit from utilizing multi-method designs, i.e., the combination of qualitative, longitudinal and cross-sectional research strategies, to gain a more comprehensive understanding of identity-relevant processes during cultural transition.

It is also important to recognize that identity processes do not operate in a vacuum, but rather that they interact with more general cognitive and affective processing (e.g., approach- 
avoidance) and other forms of psychological functioning (e.g., motivations or control mechanisms). Many researchers have argued that classification and cluster analytical techniques provide an effective tool to determine functional configurations and to examine the dynamic interplay of the operating factors (Bergman, Magnusson, \& El Khouri, 2003; Magnusson \& Allen, 1983). Similarly, it is suggested that a person-oriented approach to data analysis could offer important information about typical patterns of underlying processes that facilitate, or obstruct, optimal psychological functioning during acculturation.

On a conceptual-theoretical level, there is significant potential in expanding theoretical frameworks to accommodate the developmental perspective. The current work demonstrates how identity processing styles can be applied within the stress and coping paradigm to explain changes in the psychological wellbeing of immigrants. This was an attempt to incorporate the developmental perspective in to the $\mathrm{ABC}$ model of acculturation.

Acculturation research distinguishes between psychological and sociocultural forms of cross-cultural adjustment (Searle \& Ward, 1990; Ward et al., 2001). The current research concentrated on the psychological domain of adaptation. A next step could be to elucidate how developmental mechanisms might interplay with socio-cultural adjustment, i.e., the behavioural component of cross-cultural adaptation. It is fair to assume that information processing and decision making strategies exert a differential effect on cultural skill acquisition. For example, it is very likely that individuals with a normative style to the host society will learn the official language and the culturally appropriate behaviours much faster compared to their peers with a predominantly normative orientation to the country of origin. In a similar way, it would be worth having a closer look at the potential impact of identity strategies on immigrant identification in the framework of social identity theory (i.e., the cognitive component of adjustment). Although the studies reported in this thesis assessed identity commitment and identity outcomes in the context of migration and cultural transition, it remains a question for future research whether being an immigrant eventually evolves into a new social identity.

In addition to assessing psychological and socio-cultural adaptation, linking identity processes to indicators of behavioural and health outcomes could have important implications for professionals working with immigrant populations, thus improving practice through research. For example, previous studies have linked the diffuse-avoidant orientation to increased health problems and medical symptoms, delinquent behaviour, and substance use (Adams et al., 2001; Adams et al., 2005; Jorgensen, 2009; Wheeler, Adams, \& Keating, 2001; White \& Jones, 1996). The inability to overcome acculturation identity crises can have 
harmful effects not only for immigrants' general and mental health, but may also lead to reduced productivity, withdrawal from the labour market, or sustained reliance on the welfare and health care systems. Arguably, it is the best interest of host societies to assist newcomers with their settlement, as well as to provide appropriate support services to smooth the transition process and advance pathways to immigrant wellbeing. Along these lines, future investigations of acculturation and identity might be undertaken with an explicit objective to inform policy. Cross-cultural comparative studies could offer an idea of how the identity development of immigrants can be promoted or hindered through policies related to immigration, diversity or equality in various socio-political contexts.

\section{Concluding Comments}

Immigration is on the rise globally, leading to a marked increase in population diversity and intercultural contact in most regions of the world. Crossing cultures and moving to a new society involve a variety of psychological changes with a particular relevance to identity development. Confronting unfamiliar values and social norms, communicating using a foreign language, and interacting with members of the host culture prompt immigrants for self-examination. Identity crises commonly arise during periods of active identity exploration, which require comprehensive identity management. However, so far relatively little research has been done to examine identity management strategies among first generation, adult immigrants.

The purpose of the present research programme was to address this gap in the literature and provide a comprehensive investigation of the identity processing strategies operating during an acculturation identity crisis. By adapting Berzonsky's $(1989,2011)$ theory of identity styles to the immigrant population, I was able to analyse identity development in an integrated framework representing an intersection of acculturation and developmental research. The series of studies reported here answered important questions about the development of a committed and positively evaluated immigrant identity and contributed to our knowledge about the role of intrapsychic mechanisms, such as individual differences in information processing and decision making, in the cross-cultural adjustment of immigrants.

In conclusion, I would like to emphasize three main outcomes of this research project. First, acculturation identity crises are normative and expected periods of development for acculturating individuals. Secondly, the research presented in this thesis points out that cultural transition can lead to positive growth, if individuals in transition manage to resolve the identity stress arising from identity crisis experienced in connection with 
immigration/intercultural contact. There are, however, deeper individual differences in the ways people approach identity stress, which greatly impact whether and how successfully immigrants overcome an acculturation identity crisis. Finally, developmental theories provide useful and effective frameworks for the study of identity processes in a cross-cultural context. The integration of developmental and acculturation models offer us an unparalleled opportunity to advance our understanding of human development in context and to gain insights into aspects of cross-cultural transition that have been overlooked by acculturation researchers. 


\section{References}

Abouguendia, M., \& Noels, K. A. (2001). General and acculturation-related daily hassles and psychological adjustment in first- and second-generation South Asian immigrants to Canada. International Journal of Psychology, 36(3), 163-173. doi: $10.1080 / 741930008$

Adams, G. R., \& Marshall, S. K. (1996). A developmental social psychology of identity: Understanding the person-in-context. Journal of Adolescence, 19, 429-442.

Adams, G. R., Munro, B., Doherty-Poirer, M., Munro, G., Petersen, A.-M. R., \& Edwards, J. (2001). Diffuse-avoidance, normative, and informational identity styles: Using identity theory to predict maladjustment. Identity: An International Journal of Theory and Research, 1, 307-320.

Adams, G. R., Munro, B., Munro, G., Doherty-Poirer, M., \& Edwards, J. (2005). Identity processing styles and canadian adolescents' self-reported delinquency. Identity: An International Journal of Theory and Research, 5, 57-65.

Argyle, M. (1969). Social interaction. London: Meuthen and Co. Ltd.

Ataca, B., \& Berry, J. W. (2002). Psychological, sociocultural, and marital adaptation of Turkish immigrant couples in Canada. International Journal of Psychology, 37(1), 13 26. doi: 10.1080/00207590143000135

Aycan, Z., \& Berry, J. W. (1996). Impact of employment-related experiences on immigrants' psychological well-being and adaptation to Canada. Canadian Journal of Behavioural Science, 28(3), 240-251. doi: 10.1037/0008-400x.28.3.240

Bagozzi, R. P., \& Edwards, J. R. (1998). A general approach for rpresenting constructs in organizational research. Organizational Research Methods, 1, 45-87. doi: $10.1177 / 109442819800100104$

Baumeister, R. F., Shapiro, J. P., \& Tice, D. M. (1985). Two kinds of identity crisis. Journal of Personality 53, 407-424. doi: 10.1111/j.1467-6494.1985.tb00373.x

Beaumont, S. L. (2009). Identity processing and personal wisdom: An information-oriented identity style predicts self-actualization and self-transcendence. Identity: An International Journal of Theory and Research, 9, 95-115.

Beaumont, S. L., \& Pratt, M. M. (2011). Identity processing styles and psychosocial balance during early and middle adulthood: The role of identity in intimacy and generativity. Journal of Adult Development, 18(4), 172-183. doi: 10.1007/s10804-011-9125-Z

Beaumont, S. L., \& Scammell, J. (2012). Patterns of spirituality and meaning in life related to identity. Identity: An International Journal of Theory and Research, 12, 345-367. 
Beaumont, S. L., \& Seaton, C. L. (2011). Patterns of coping associated with identity processing styles. Identity: An International Journal of Theory and Research, 11, 348361. doi: 10.1080/15283488.2011.613590

Benet-Martinez, V., \& Haritatos, J. (2005). Bicultural identity integration (BII): Components and psychosocial antecedents. Journal of Personality, 73(4), 1015-1049. doi: 10.1111/j.1467-6494.2005.00337.x

Bergman, L. R., Magnusson, D., \& El Khouri, B. M. (2003). Studying individual development in an interindividual context: A person-oriented approach (paths through life series) Mahwah, NJ: Erlbaum.

Berjot, S., Altintas, E., Lesage, F.-X., \& Grebot, E. (2013). The impact of work stressors on identity threats and perceived stress: An exploration of sources of difficulty at work among French psychologists. Sage Open, 3(1-11). doi: 10.1177/2158244013505292

Berjot, S., Girault-Lidvan, N., \& Gillet, N. (2012). Appraising stigmatization and discrimination: Construction and validation of a questionnaire assessing threat and challenge appraisals to personal and social identity. Identity: An International Journal of Theory and Research, 12, 191-216.

Berman, A. M., Schwartz, S. J., Kurtines, W. M., \& Berman, S. L. (2001). The process of exploration in identity formation: The role of style and competence. Journal of Adolescence, 24, 513-528. doi: 10.1006/jado.2001.0386

Berry, J. W. (1990). Psychology of acculturation. In R. W. Brislin (Ed.), Applied crosscultural psychology (pp. 232-253). Newbury Park, CA: Sage.

Berry, J. W. (2005). Acculturation: Living successfully in two cultures. International Journal of Intercultural Relations, 29(6), 697-712. doi: DOI 10.1016/j.ijintrel.2005.07.013

Berry, J. W. (2006). Stress perspective on acculturation. In D. L. Sam \& J. W. Berry (Eds.), The Cambridge handbook of acculturation psychology (pp. 43-57). UK: Cambridge University Press.

Berry, J. W. (2009). A critique of critical acculturation. International Journal of Intercultural Relations, 33(5), 361-371. doi: 10.1016/j.ijintrel.2009.06.003

Berry, J. W., Phinney, J. S., Sam, D. L., \& Vedder, P. (2006). Immigrant youth: Acculturation, identity, and adaptation. Applied Psychology: An International Review, 55(3), 303-332. doi: 10.1111/j.1464-0597.2006.00256.x

Berry, J. W., \& Sabatier, C. (2010). Acculturation, discrimination, and adaptation among second generation immigrant youth in Montreal and Paris. International Journal of Intercultural Relations, 34(3), 191-207. doi: 10.1016/j.ijintrel.2009.11.007 
Berzonsky, M. D. (1989). Identity style: Conceptualization and measurement. Journal of Adolescent Research, 4, 268-282.

Berzonsky, M. D. (1992a). Identity style and coping strategies. Journal of Personality 60(4), 771-788.

Berzonsky, M. D. (1992b). Identity style inventory (ISI-3): Revised version. Unpublished Measure. State University of New York. Department of Psychology, State University of New York, Cortland, NY.

Berzonsky, M. D. (2003). Identity style and well-being: Does commitment matter? Identity: An International Journal of Theory and Research, 3, 131-142.

Berzonsky, M. D. (2008). Identity formation: The role of identity processing style and cognitive processes. Personality and Individual Differences, 44(3), 645-655. doi: 10.1016/j.paid.2007.09.024

Berzonsky, M. D. (2011). A social-cognitive perspective on identity construction. In S. J. Schwartz, K. Luyckx \& V. L. Vignoles (Eds.), Handbook of identity theory and research. New York: Springer.

Berzonsky, M. D., \& Adams, G. R. (1999). Reevaluating the identity status paradigm: Still useful after 35 years. Developmental Review, 19(4), 557-590. doi:

10.1006/drev.1999.0495

Berzonsky, M. D., Cieciuch, J., Duriez, B., \& Soenens, B. (2011). The how and what of identity formation: Associations between identity styles and value orientations. Personality and Individual Differences, 50(2), 295-299. doi: 10.1016/j.paid.2010.10.007

Berzonsky, M. D., \& Ferrari, J. R. (1996). Identity orientation and decisional strategies. Personality and Individual Differences, 20(5), 597-606. doi: 10.1016/01918869(96)00001-3

Berzonsky, M. D., \& Ferrari, J. R. (2009). A diffuse-avoidant identity processing style: Strategic avoidance or self-confusion? Identity: An International Journal of Theory and Research, 9, 145-158.

Berzonsky, M. D., \& Kuk, L. S. (2000). Identity status, identity processing style, and the transition to university. Journal of Adolescent Research, 15(1), 81-98. doi: 10.1177/0743558400151005

Berzonsky, M. D., \& Kuk, L. S. (2005). Identity style, psychosocial maturity, and academic performance. Personality and Individual Differences, 39(1), 235-247. doi:

10.1016/j.paid.2005.01.010 
Berzonsky, M. D., \& Luyckx, K. (2008). Identity styles, self-reflective cognition, and identity processes: A study of adaptive and maladaptive dimensions of self-analysis. Identity: An International Journal of Theory and Research, 8, 205-219.

Berzonsky, M. D., Macek, P., \& Nurmi, J. E. (2003). Interrelationships among identity process, content, and structure: A cross-cultural investigation. Journal of Adolescent Research, 18(2), 112-130. doi: 10.1177/0743558402250344

Berzonsky, M. D., \& Neimeyer, G. J. (1994). Ego identity status and identity processing orientation - the moderating role of commitment. Journal of Research in Personality, 28(4), 425-435. doi: DOI 10.1006/jrpe.1994.1030

Berzonsky, M. D., Soenens, B., Luyckx, K., Smits, I., Papini, D. R., \& Goossens, L. (2013). Development and validation of the revised identity style inventory (ISI-5): factor structure, reliability, and validity. Psychological Assessment, 25(3), 893-904. doi: $10.1037 / \mathrm{a} 0032642$

Berzonsky, M. D., \& Sullivan, C. (1992). Social-cognitive aspects of identity style: Need for cognition, experiemtial openness, and introspection. Journal of Adolescent Research, 7, 140-155. doi: 10.1177/074355489272002

Birman, D., Trickett, E. J., \& Vinokurov, A. (2002). Acculturation and adaptation of Soviet Jewish refugee adolescents: Predictors of adjustment across life domains. American Journal of Community Psychology, 30(5), 585-607. doi: UNSP pp545-ajcp-376933

DOI 10.1023/A:1016323213871

Bosch, L. A., \& Card, N. A. (2012). A meta-analytic review of Berzonsky's Identity Style Inventory (ISI). Journal of Adolescence, 35(2), 333-343. doi: 10.1016/j.adolescence.2011.08.007

Bosch, L. A., Segrin, C., \& Curran, M. (2012). Identity Style during the transition to adulthood: The role of family communication patterns, perceived support, and affect. Identity: An International Journal of Theory and Research, 12, 275-295. doi: $10.1080 / 15283488.2012 .716379$

Boski, P. (2013). A Psychology of Economic Migration. Journal of Cross-Cultural Psychology, 44(7), 1067-1093. doi: 10.1177/0022022112471895

Bosma, H. A., \& Kunnen, E. S. (2001). Determinants and mechanisms in ego identity development: A review and synthesis. Developmental Review, 21(1), 39-66. doi: 10.1006/drev.2000.0514 
Briggs, S. R., \& Cheek, J. M. (1986). The role of factor-analysis in the development and evaluation of personality scales. Journal of Personality, 54(1), 106-148. doi: 10.1111/j.1467-6494.1986.tb00391.x

Bronfenbrenner, U., \& Morris, P. A. (2006). The bioecological model of human development. In W. Damon \& R. M. Lerner (Eds.), Handbook of child psychology: Vol. 1. theoretical models of human development (pp. 793-828). Hoboken, NJ: Wiley.

Brubaker, R., \& Cooper, F. (2000). Beyond "identity". Theory and Society, 29(1), 1-47. doi: 10.1023/A:1007068714468

Cacioppo, J. T., \& Petty, R. E. (1982). The need for cognition. Journal of Personality and Social Psychology, 42(1), 116-131. doi: 10.1037//0022-3514.42.1.116

Campbell, D. T., \& Fiske, D. W. (1959). Convergent and discriminant validation by the multitrait-multimethod matrix. Psychological Bulletin, 56, 81-105. doi: $10.1037 / \mathrm{H} 0046016$

Campbell, J. D., Trapnell, P. D., Heine, S. J., Katz, I. M., Lavallee, L. F., \& Lehman, D. R. (1996). Self-concept clarity: Measurement, personality correlates, and cultural boundaries. Journal of Personality and Social Psychology, 70, 1114-1114.

Chen, S. X., Benet-Martinez, V., \& Bond, M. H. (2008). Bicultural identity, bilingualism, and psychological adjustment in multicultural societies: Immigration-based and globalization-based acculturation. Journal of Personality, 76(4), 803-837. doi: 10.1111/j.1467-6494.2008.00505.x

Chirkov, V. (2009). Critical psychology of acculturation: What do we study and how do we study it, when we investigate acculturation? International Journal of Intercultural Relations, 33(2), 94-105. doi: 10.1016/j.ijintrel.2008.12.004

Chung, R. H., Kim, B. S., \& Abreu, J. M. (2004). Asian American multidimensional acculturation scale: development, factor analysis, reliability, and validity. Cultur Divers Ethnic Minor Psychol, 10(1), 66-80. doi: 10.1037/1099-9809.10.1.66

Clark, L. A., \& Watson, D. (1995). Constructing validity: Basic issues in objective scale development. Psychological Assessment, 7, 309-319. doi: Doi 10.1037//10403590.7.3.309

Compas, B. E., Connor-Smith, J. K., Saltzman, H., Thomsen, A. H., \& Wadsworth, M. E. (2001). Coping with stress during childhood and adolescence: problems, progress, and potential in theory and research. Psychological Bulletin, 127, 87-127. 
Connor-Smith, J. K., \& Compas, B. E. (2004). Coping as a moderator of relations between reactivity to interpersonal stress, health status, and internalizing problems. Cognitive Therapy and Research, 28, 347-368.

Costigan, C. L., Koryzma, C. M., Hua, J. M., \& Chance, L. J. (2010). Ethnic identity, achievement, and psychological adjustment: Examining risk and resilience among youth from immigrant chinese families in Canada. Cultural Diversity \& Ethnic Minority Psychology, 16, 264-273. doi: 10.1037/A0017275

Cote, J. E. (1997). An empirical test of the identity capital model. Journal of Adolescence, 20(5), 577-597. doi: 10.1006/jado.1997.0111

Côté, J. E., \& Levine, C. (1987). Aformulation of Erikson's theory of ego identity formation. Developmental Review, 7, 273-325.

Crocetti, E., Rubini, M., Berzonsky, M. D., \& Meeus, W. (2009). Brief report: the identity style inventory - validation in Italian adolescents and college students. Journal of Adolescence, 32(2), 425-433. doi: 10.1016/j.adolescence.2008.04.002

Crocetti, E., \& Shokri, O. (2010). Iranian validation of the identity style inventory. International Journal of Testing, 10, 185-199.

Crockett, L. J., Iturbide, M. I., Stone, R. A. T., McGinley, M., Raffaelli, M., \& Carlo, G. (2007). Acculturative stress, social support, and coping: Relations to psychological adjustment among Mexican American college students. Cultural Diversity \& Ethnic Minority Psychology, 13(4), 347-355. doi: 10.1037/1099-9809.13.4.347

Cuéllar, I., Harris, L. C., \& Jasso, R. (1980). An acculturation scale for Mexican-Americans normal and clinical populations. Hispanic Journal of Behavioral Sciences, 2, 199217.

Dimitrova, R., Bender, M., Chasiotis, A., \& van de Vijver, F. J. R. (2013). Ethnic identity and acculturation of Turkish-Bulgarian adolescents. International Journal of Intercultural Relations, 37(1), 1-10. doi: 10.1016/j.ijintrel.2012.04.005

Dollinger, S. M. C. (1995). Identity styles and the five-factor model of personality. Journal of Research in Personality, 29(4), 475-479. doi: 10.1006/jrpe.1995.1028

Doumen, S., Smits, I., Luyckx, K., Duriez, B., Vanhalst, J., Verschueren, K., \& Goossens, L. (2012). Identity and perceived peer relationship quality in emerging adulthood: The mediating role of attachment-related emotions. Journal of Adolescence, 35(6), $1417-$ 1425. doi: 10.1016/j.adolescence.2012.01.003 
Duriez, B., Smits, I., \& Goossens, L. (2008). The relation between identity styles and religiosity in adolescence: Evidence from a longitudinal perspective. Personality and Individual Differences, 44(4), 1022-1031. doi: 10.1016/j.paid.2007.10.028

Duriez, B., \& Soenens, B. (2006). Personality, identity styles and authoritarianism: An integrative study among late adolescents. European Journal of Personality, 20(5), 397-417. doi: 10.1002/Per.589

Duriez, B., Soenens, B., \& Beyers, W. (2004). Personality, identity styles, and religiosity: An integrative study among late adolescents in Flanders (Belgium). Journal of Personality, 72(5), 877-910. doi: 10.1111/j.0022-3506.2004.00284.x

Epstein, S., Pacini, R., Denes-Raj, V., \& Heier, H. (1996). Individual differences in intuitiveexperiential and analytical-rational thinking styles. Journal of Personality and Social Psychology, 71(2), 390-405. doi: 10.1037/0022-3514.71.2.390

Erikson, E. H. (1950). Childhood and society (1st ed.). New York: Norton.

Erikson, E. H. (1968). Identity, youth, and crisis (1st ed.). New York: Norton.

Eryigit, S. (2010). Identity formation in context. (PhD), Auburn University, Auburn, Alabama.

Eryigit, S., \& Kerpelman, J. (2009). Using the identity processing style Q-sort to examine identity styles of Turkish young adults. Journal of Adolescence, 32(5), 1137-1158. doi: 10.1016/j.adolescence.2009.02.001

Fadjukoff, P., Pulkkinen, L., \& Kokko, K. (2005). Identity processes in adulthood: Diverging domains. Identity: An International Journal of Theory and Research, 5, 1-20.

Faramarzi, M., Jahanian, K., Zarbakhsh, M., Salehi, S., \& Pasha, H. (2014). The role of moral intelligence and identity styles in prediction of mental health problems in healthcare students. Health, 6, 664-672.

Florian, V., Mikulincer, M., \& Taubman, O. (1995). Does hardiness contribute to mentalhealth during a stressful real-life situation? The roles of appraisal and coping. Journal of Personality and Social Psychology, 68(4), 687-695. doi: 10.1037/00223514.68.4.687

Folkman, S., \& Lazarus, R. S. (1980). An analysis of coping in a middle-aged community sample. Journal of Health and Social Behavior, 21(3), 219-239. doi: 10.2307/2136617

Folkman, S., Lazarus, R. S., Gruen, R. J., \& Delongis, A. (1986). Appraisal, coping, health status, and psychological symptoms. Journal of Personality and Social Psychology, 50(3), 571-579. doi: 10.1037//0022-3514.50.3.571 
Forsythe, C. J., \& Compas, B. E. (1987). Interaction of cognitive appraisals of stressful events and coping: Testing the goodness of fit hypothesis. Cognitive Therapy and Research, 11(4), 473-485. doi: 10.1007/Bf01175357

Galchenko, I., \& van de Vijver, F. J. R. (2007). The role of perceived cultural distance in the acculturation of exchange students in Russia. International Journal of Intercultural Relations, 31(2), 181-197. doi: 10.1016/j.ijintrel.2006.03.004

Ghavami, N., Fingerhut, A., Peplau, L. A., Grant, S. K., \& Wittig, M. A. (2011). Testing a model of minority identity achievement, identity affirmation, and psychological wellbeing among ethnic minority and sexual minority individuals. Cultural Diversity \& Ethnic Minority Psychology, 17(1), 79-88. doi: 10.1037/A0022532

Giancola, J. K., Grawitch, M. J., \& Borchert, D. (2009). Dealing with the stress of college: A model for adult students. Adult Education Quarterly, 59(3), 246-263. doi: $10.1177 / 0741713609331479$

Graves, T. D. (1967). Psychological acculturation in a tri-ethnic community. Southwestern Journal of Anthropology, 23(4), 337-350.

Gray-Little, B., Williams, V. S. L., \& Hancock, T. D. (1997). An item response theory analysis of the Rosenberg self-esteem scale. Personality and Social Psychology Bulletin, 23(5), 443-451. doi: 10.1177/0146167297235001

Harvey, A., Nathens, A. B., Bandiera, G., \& LeBlanc, V. R. (2010). Threat and challenge: cognitive appraisal and stress responses in simulated trauma resuscitations. Medical Education, 44(6), 587-594. doi: 10.1111/j.1365-2923.2010.03634.x

Heron, A. \& Kroeger, E. (1981). Introduction to developmental psychology. In H.C. Triandis, \& A. Heron (Eds.), Handbook of cross-cultural psychology. Vol. IV. (pp. 1-15). Boston, MA: Allyn \& Bacon.

Holmes, T. H., \& Rahe, R. H. (1967). Social Readjustment Rating Scale. Journal of Psychosomatic Research, 11(2), 213-\&. doi: 10.1016/0022-3999(67)90010-4

Hong, Y. Y., Morris, M. W., Chiu, C. Y., \& Benet-Martinez, V. (2000). Multicultural minds: A dynamic constructivist approach to culture and cognition. American Psychologist, 55(7), 709-720. doi: 10.1037//0003-066x.55.7.709

Hu, L. T., \& Bentler, P. M. (1999). Cutoff criteria for fit indexes in covariance structure analysis: Conventional criteria versus new alternatives. Structural Equation Modeling: A Multidisciplinary Journal, 6(1), 1-55. doi: 10.1080/10705519909540118 
Jasinskaja-Lahti, I., \& Liebkind, K. (2001). Perceived discrimination and psychological adjustment among Russian-speaking immigrant adolescents in Finland. International Journal of Psychology, 36(3), 174-185.

Jibeen, T., \& Khalid, R. (2010). Predictors of psychological well-being of Pakistani immigrants in Toronto, Canada. International Journal of Intercultural Relations, 34(5), 452-464. doi: 10.1016/j.ijintrel.2010.04.010

Johnson, E. A., \& Nozick, K. J. (2011). Personality, adjustment, and identity style influences on stability in identity and self-concept during the transition to university. Identity: An International Journal of Theory and Research, 11, 25-46.

Jorgensen, C. R. (2009). Identity style in patients with borderline personality disorder and normal controls. Journal of Personality Disorders, 23(2), 101-112.

Jose, P. E. (2013). ModGraph-I: A programme to compute cell means for the graphical display of moderational analyses: The internet version, Version 3.0. Wellington, New Zealand: Victoria University of Wellington. Retrieved from http://pavlov.psyc.vuw.ac.nz/paul-jose/modgraph/

Kaplan, R. M., \& Saccuzzo, D. P. (1982). Psychological testing: principles, applications, and issues. Monterey, Calif.: Brooks/Cole Pub. Co.

Kim, B. S. K., \& Omizo, M. M. (2005). Asian and European American cultural values, collective self-esteem, acculturative stress, cognitive flexibility, and general selfefficacy among Asian American college students. Journal of Counseling Psychology, 52(3), 412-419. doi: 10.1037/0022-0167.52.3.412

King, M. F., \& Bruner, G. C. (2000). Social desirability bias: A neglected aspect of validity testing. Psychology \& Marketing, 17(2), 79-103. doi: 10.1002/(Sici)15206793(200002)17:2<79::Aid-Mar2>3.0.Co;2-0

Konig, J. (2009). Moving Experience: Dialogues between Personal Cultural Positions. Culture \& Psychology, 15(1), 97-119. doi: 10.1177/1354067x08099617

Kosmitzki, C. (1996). The reaffirmation of cultural identity in cross-cultural encounters. Personality and Social Psychology Bulletin, 22(3), 238-248. doi:

$10.1177 / 0146167296223002$

Krettenauer, T. (2005). The role of epistemic cognition in adolescent identity formation: Further evidence. Journal of Youth and Adolescence, 34(3), 185-198. doi: $10.1007 / \mathrm{s} 10964-005-4300-9$ 
Krishnan, A., \& Berry, J. W. (1992). Acculturative stress and acculturation attitudes among Indian Immigrants to the United States. Psychology \& Developing Societies, 4, 187212.

Lackzo, F., \& Appave, G. (2014). World migration report 2013: Migrant well-being and development. Geneva.

Lazarus, R. S., \& Folkman, S. (1984). Stress, appraisal and coping. New York: Springer.

Lee, J. S., Koeske, G. F., \& Sales, E. (2004). Social support buffering of acculturative stress: a study of mental health symptoms among Korean international students. International Journal of Intercultural Relations, 28(5), 399-414. doi: 10.1016/j.ijintrel.2004.08.005

Leong, C. H., \& Ward, C. (2000). Identity conflict in sojourners. International Journal of Intercultural Relations, 24(6), 763-776. doi: 10.1016/S0147-1767(00)00030-4

Liebkind, K. (2006). Ethnic identity and acculturation. In D. L. Sam \& J. W. Berry (Eds.), The Cambridge handbook of acculturation psychology (pp. 78-96). New York: Cambridge University Press.

Lim, K. V., Heiby, E., Brislin, R., \& Griffin, B. (2002). The development of the Khmer acculturation scale. International Journal of Intercultural Relations, 26(6), 653-678. doi: Pii S0147-1767(02)00040-8

Little, T. D., Cunningham, W. A., Shahar, G., \& Widaman, K. F. (2002). To parcel or not to parcel: Exploring the question, weighing the merits. Structural Equation Modeling, 9(2), 151-173. doi: 10.1207/S15328007sem0902_1

Luyckx, K., Klimstra, T. A., Duriez, B., Van Petegem, S., \& Beyers, W. (2013). Personal identity processes from adolescence through the late 20s: Age trends, functionality, and depressive symptoms. Social Development, 22, 701-721. doi: 10.1111/sode.12027

Luyckx, K., Lens, W., Smits, I., \& Goossens, L. (2010). Time perspective and identity formation: Short-term longitudinal dynamics in college students. International Journal of Behavioral Development, 34(3), 238-247. doi: 10.1177/0165025409350957

Luyckx, K., Soenens, B., Berzonsky, M. D., Smas, I., Goossens, L., \& Vansteenkiste, M. (2007). Information-oriented identity processing, identity consolidation, and wellbeing: The moderating role of autonomy, self-reflection, and self-rumination. Personality and Individual Differences, 43(5), 1099-1111. doi: 10.1016/j.paid.2007.03.003

Magnusson, D., \& Allen, V. L. (1983). Human development: An interactional perspective. New York, NY: Academic Press. 
Mak, A. S., Blewitt, K., \& Heaven, P. C. L. (2004). Gender and personality influences in adolescent threat and challenge appraisals and depressive symptoms. Personality and Individual Differences, 36(6), 1483-1496. doi: 10.1016/S0191-8869(03)00243-5

Mak, A. S., \& Neil, A. L. (2006). Prevention, protection and promotion. Proceedings of the Second International Asian Health and Wellbeing Conference. Paper presented at the The second international Asian health and wellbeing conference: Precention, protection and promotion, Auckland, NZ.

Marcia, J. E. (1966). Development and validation of ego-identity status. Journal of Personality and Social Psychology, 3(5), 551-\&. doi: 10.1037/H0023281

Miklikowska, M. (2012). Psychological underpinnings of democracy: Empathy, authoritarianism, self-esteem, interpersonal trust, normative identity style, and openness to experience as predictors of support for democratic values. Personality and Individual Differences, 53(5), 603-608. doi: 10.1016/j.paid.2012.04.032

United Nations (2013). International migration report 2013. New York.

Navara, G. S., \& James, S. (2002). Sojourner adjustment: does missionary status affect acculturation? International Journal of Intercultural Relations, 26(6), 695-709. doi: Pii S0147-1767(02)00042-1

Nguyen, A. M. D., \& Benet-Martinez, V. (2013). Biculturalism and adjustment: A metaanalysis. Journal of Cross-Cultural Psychology, 44, 122-159. doi: $10.1177 / 0022022111435097$

Nguyen, H. H., Messe, L. A., \& Stollak, G. E. (1999). Toward a more complex understanding of acculturation and adjustment - Cultural involvements and psychosocial functioning in Vietnamese youth. Journal of Cross-Cultural Psychology, 30(1), 5-31. doi: $10.1177 / 0022022199030001001$

Nunnally, J. C., \& Bernstein, I. H. (1994). Psychometric theory (3rd ed.). New York: McGraw-Hill.

Nunnaly, J. C. (1978). Psychometric theory (2nd ed.). New York: McGraw-Hill.

Nurmi, J. E., Berzonsky, M. D., Tammi, K., \& Kinney, A. (1997). Identity processing orientation, cognitive and behavioural strategies and well-being. International Journal of Behavioral Development, 21(3), 555-570.

OECD. (2013). World migration in figures. Retrieved 01/06/2014, from http://www.oecd.org/els/mig/World-Migration-in-Figures.pdf 
Oetting, E. R., \& Beauvais, F. (1991). Orthogonal cultural identification theory: The cultural identification of minority adolescents. International Journal of the Addictions, 25(5A6A), 655-685.

Pakenham, K. I., \& Rinaldis, M. (2001). The role of illness, resources, appraisal, and coping strategies in adjustment to HIV/AIDS: The direct and buffering effects. Journal of Behavioral Medicine, 24(3), 259-279. doi: 10.1023/A:1010718823753

Pan, J. Y. (2008). Acculturation and resilience of mainland Chinese postgraduate students in Hong Kong. (PhD), University of Hong Kong, Hong Kong.

Pan, J. Y. (2011). A resilience-based and meaning-oriented model of acculturation: A sample of mainland Chinese postgraduate students in Hong Kong. International Journal of Intercultural Relations, 35(5), 592-603. doi: 10.1016/j.ijintrel.2011.02.009

Park, C. L., Armeli, S., \& Tennen, H. (2004). Appraisal-coping goodness of fit: A daily Internet study. Personality and Social Psychology Bulletin, 30(5), 558-569. doi: $10.1177 / 0146167203262855$

Passmore, N. L., Fogarty, G. J., Bourke, C. J., \& Baker-Evans, S. F. (2005). Parental bonding and identity style as correlates of self-esteem among adult adoptees and nonadoptees. Family Relations, 54(4), 523-534. doi: 10.1111/j.1741-3729.2005.00338.x

Pellegrino Aveni, V. A. (2005). Study abroad and second language use: Constructing the self. Cambridge: Cambridge University Press.

Phinney, J. S. (1989). Stages of ethnic identity development in minority group adolescence. The Journal of Early Adolescence, 9, 34-49.

Phinney, J. S. (1990). Ethnic identity in adolescents and adults: review of research. Psychological Bulletin, 108(3), 499-514. doi: 10.1037/0033-2909.108.3.499

Phinney, J. S. (1991). Ethnic identity and self-esteem: A review and integration. Hispanic Journal of Behavioral Sciences, 13(2), 193-208. doi: 10.1177/07399863910132005

Phinney, J. S. (1992). The multigroup ethnic identity measure: A new scale for use with diverse groups. Journal of Adolescent Research, 7, 156-176.

Phinney, J. S., \& Ong, A. D. (2007). Conceptualization and measurement of ethnic identity: Current status and future directions. Journal of Counseling Psychology, 54(3), 271281. doi: 10.1037/0022-0167.54.3.271

Pittman, J. F., Kerpelman, J. L., Lamke, L. K., \& Sollie, D. L. (2009). Development and validation of a Q-sort measure of identity processing style: The identity processing style Q-sort. Journal of Adolescence, 32(5), 1239-1265. doi: 10.1016/j.adolescence.2009.01.002 
Raftery, A. E. (1995). Bayesian model selection in social research. Sociological Methodology, 25, 111-163.

Redfield, R., Linton, R., \& Herskovits, M. (1936). A memorandum for the study of acculturation. American Anthropologist, 38, 149-152.

Reynolds, W. M. (1982). Development of reliable and valid short forms of the MarloweCrowne social desirability scale. Journal of Clinical Psychology, 38(1), 119-125. doi: 10.1002/1097-4679(198201)38:1<119::Aid-Jclp2270380118>3.0.Co;2-I

Roberts, R., Phinney, J. S., Masse, L., Chen, Y., Roberts, C., \& Romero, A. (1999). The structure of ethnic identity in young adolescents from diverse ethnocultural groups. Journal of Early Adolescence, 19, 301-322.

Roets, A., \& Van Hiel, A. (2011). Item selection and validation of a brief, 15-item version of the Need for Closure Scale. Personality and Individual Differences, 50(1), 90-94. doi: 10.1016/j.paid.2010.09.004

Rosenberg, M. (1965). Society and the adolescent self-image. Princeton, N.J.,: Princeton University Press.

Ryder, A. G., Alden, L. E., \& Paulhus, D. L. (2000). Is acculturation unidimensional or bidimensional? A head-to-head comparison in the prediction of personality, selfidentity, and adjustment. Journal of Personality and Social Psychology, 79(1), 49-65. doi: 10.1037//0022.3514.79.1.49

Safdar, S., Lewis, J. R., \& Daneshpour, M. (2006). Social axioms in Iran and Canada: Intercultural contact, coping and adjustment. Asian Journal of Social Psychology, 9(2), 123-131. doi: 10.1111/j.1467-839X.2006.00189.x

Sayegh, L., \& Lasry, J. C. (1993). Immigrants adaptation in Canada: Assimilation, acculturation, and orthogonal cultural identification. Canadian PsychologyPsychologie Canadienne, 34(1), 98-109. doi: 10.1037/H0078777

Schwartz, A. L., Galliher, R. V., \& Rodriguez, M. M. D. (2011). Self-disclosure in Latinos' intercultural and intracultural friendships and acquaintanceships: Links with collectivism, ethnic identity, and acculturation. Cultural Diversity \& Ethnic Minority Psychology, 17(1), 116-121. doi: 10.1037/A0021824

Schwartz, S. J. (2001). The evolution of Eriksonian and Neo-Eriksonian identity theory and research: A review and integration. Identity: An International Journal of Theory and Research, 1, 7-58. 
Schwartz, S. J. (2005). A new identity for identity research: Recommendations for expanding and refocusing the identity literature. Journal of Adolescent Research, 20(3), 293-308. doi: $10.1177 / 0743558405274890$

Schwartz, S. J., Cote, J. E., \& Arnett, J. J. (2005). Identity and agency in emerging adulthood: Two development routes in the individualization process. Youth \& Society, 37(2), 201-229. doi: 10.1177/0044118x05275965

Schwartz, S. J., Klimstra, T. A., Luckx, K., Hale W. W., Meeus, W. H. J. (2012). Characterizing the self-system over time in adolescence: Internal structure and associations with internalizing symptoms. Journal of Youth anmd Adolescence, 41, 1208-1221. doi: 10.1007/s10964-012-9751-1

Schwartz, S. J., \& Montgomery, M. J. (2002). Similarities or differences in identity development? The impact of acculturation and gender on identity process and outcome. Journal of Youth and Adolescence, 31(5), 359-372. doi: 10.1023/A:1015628608553

Schwartz, S. J., Montgomery, M. J., \& Briones, E. (2006). The role of identity in acculturation among immigrant people: Theoretical propositions, empirical questions, and applied recommendations. Human Development, 49, 1-30.

Searle, W., \& Ward, C. (1990). The prediction of psychological and sociocultural adjustment during cross-cultural transitions. International Journal of Intercultural Relations, 14(4), 449-464. doi: 10.1016/0147-1767(90)90030-Z

Shrout, P. E., \& Lane, S. P. (2012). Reliability. In H. Cooper (Ed.), APA Handbook of Research Methods in Psychology: Vol. 1. Foundations, Planning, Measures, and Psychometrics. Washington: American Psychological Association.

Skinner, N., \& Brewer, N. (2002). The dynamics of threat and challenge appraisals prior to stressful achievement events. Journal of Personality and Social Psychology, 83(3), 678-692. doi: 10.1037//0022-3514.83.3.678

Smith, R. A., \& Khawaja, N. G. (2011). A review of the acculturation experiences of international students. International Journal of Intercultural Relations, 35(6), 699713. doi: 10.1016/j.ijintrel.2011.08.004

Smits, I. (2009). Identity styles in adolescence: Measurement and associations with perceived parenting, personal well-being, and interpersonal functioning. Katholike Universiteit Leuven, Leuven.

Smits, I., Soenens, B., Vansteenkiste, M., Luyckx, K., \& Goossens, L. (2010). Why do adolescents gather information or stick to parental norms? Examining autonomous 
and controlled motives behind adolescents' identity style. Journal of Youth and Adolescence, 39(11), 1343-1356. doi: 10.1007/s10964-009-9469-х

Snauwaert, B., Soenens, B., Vanbeselaere, N., \& Boen, F. (2003). When integration does not necessarily imply integration: Different conceptualizations of acculturation orientations lead to different classifications. Journal of Cross-Cultural Psychology, 34(2), 231-239. doi: 10.1177/0022022102250250

Sochos, A., \& Diniz, M. (2012). The role of attachment in immigrant sociocultural adaptation and psychological distress. Journal of Community \& Applied Social Psychology, 22(1), 75-91. doi: 10.1002/Casp.1102

Soenens, B., \& Beaumont, S. L. (2008). Individual differences in identity styles predict proactive forms of positive adjustment. Identity: An International Journal of Theory and Research, 8, 249-268. doi: 10.1080/15283480802181891

Soenens, B., Berzonsky, M. D., Dunkel, C. S., Papini, D. R., \& Vansteenkiste, M. (2011). Are all identity commitments created equally? The importance of motives for commitment for late adolescents' personal adjustment. International Journal of Behavioral Development, 35(4), 358-369. doi: 10.1177/0165025411405954

Soenens, B., Duriez, B., \& Goossens, L. (2005). Social-psychological profiles of identity styles: attitudinal and social-cognitive correlates in late adolescence. Journal of Adolescence, 28(1), 107-125. doi: 10.1016/j.adoleseence.2004.07.001

Statistics New Zealand. (2014). 2013 census quickstats about cultural diversity. Retrieved 10.10.2014, from http://www.stats.govt.nz/Census/2013-census/profile-and-summaryreports/quickstats-about-national-highlights/cultural-diversity.aspx

Stephan, W., Ybarra, O., \& Bachman, G. (1999). Prejudice towards immigrants: An integrated threat theory. Journal of Applied Social Psychology, 29, 2221-2237.

Stiglitz, J. E., Sen, A., \& Fitoussi, J.-P. (2009). Report by the commission on the measurement of economic performance and social progress.

Suinn, R. M., Ahuna, C., \& Khoo, G. (1992). The Suinn-Lew Asian self-identity acculturation scale: Concurrent and factorial validation. Educational and Psychological Measurement, 52(4), 1041-1046. doi: 10.1177/0013164492052004028

Sussman, N. M. (2000). The dynamic nature of cultural identity throughout cultural transitions: Why home is not so sweet. Personality and Social Psychology Review, 4(4), 355-373. doi: Doi 10.1207/S15327957pspr0404_5

Syed, M., \& Azmitia, M. (2009). Longitudinal trajectories of ethnic identity during the college years. Journal of Research on Adolescence, 19(4), 601-624. 
Syed, M., Walker, L. H. M., Lee, R. M., Umana-Taylor, A. J., Zamboanga, B. L., Schwartz, S. J., . . Huynh, Q. L. (2013). A two-factor model of ethnic identity exploration: Implications for identity coherence and well-being. Cultural Diversity \& Ethnic Minority Psychology, 19(2), 143-154. doi: 10.1037/A0030564

Szabo, A., \& Ward, C. (2015). Identity development during cultural transition: The role of social-cognitive identity processes. International Journal of Intercultural Relations. doi: 10.1016/j.ijintrel.2015.03.019

Szabo, A., Ward, C., \& Fletcher, G. (2015). Identity processing styles during cultural transition: Construct and measurement. Manuscript submitted for publication.

Szabo, A., Ward, C., \& Jose, P. E. (in press). Uprooting stress, coping and anxiety: A longitudinal study of international students. International Journal of Stress Management.

Tabachnick, B. G., \& Fidell, L. S. (2007). Using multivariate statistics (5th ed.). Boston: Pearson/Allyn \& Bacon.

Tajfel, H. (1978). Differentiation between social groups: Studies in the social psychology of intergroup relations. London: Academic Press.

Tajfel, H., \& Turner, J. C. (1986). The social identity theory of intergroup behavior. In S. Worchel \& W. Austin (Eds.), Psychology of intergroup relations (pp. 7-24). Chicago: Nelson-Hall.

Thurber, C. A., \& Weisz, J. R. (1997). "You can try or you can just give up": The impact of perceived control and coping style on childhood homesickness. Developmental Psychology, 33(3), 508-517. doi: 10.1037/0012-1649.33.3.508

Turner, J. C., Hogg, M. A., Oakes, P. J., Reicher, S. D., Wetherell, M. S. (1987). Rediscovering the social group: A self-categorization theory. Oxford: Blackwell.

Turner, J. C., Oakes, P. J., Haslam, S. A., \& Mcgarty, C. (1994). Self and collective cognition and social-context. Personality and Social Psychology Bulletin, 20(5), 454463. doi: 10.1177/0146167294205002

Umaña-Taylor, A. J. (2001). Ethnic identity. In S. J. Schwartz, K. Luyckx \& V. L. Vignoles (Eds.), Handbook of Identity Theory and Research (pp. 791-809). London: Springer.

Umana-Taylor, A. J., Gonzales-Backen, M. A., \& Guimond, A. B. (2009). Latino adolescents' ethnic identity: Is there a developmental progression and does growth in ethnic identity predict growth in self-esteem? Child Development, 80(2), 391-405. doi: 10.1111/j.1467-8624.2009.01267.x 
Umaña-Taylor, A. J., \& Shin, N. (2007). An examination of ethnic identity and self-esteem with diverse populations: exploring variation by ethnicity and geography. Cultural Diversity \& Ethnic Minority Psychology, 13, 178-186.

Umana-Taylor, A. J., \& Updegraff, K. A. (2007). Latino adolescents' mental health: Exploring the interrelations among discrimination, ethnic identity, cultural orientation, self-esteem, and depressive symptoms. Journal of Adolescence, 30(4), 549-567. doi: 10.1016/j.adolescence.2006.08.002

Umaña-Taylor, A. J., Yazedjian, A., \& Bámaca-Gómez, M. (2004). Developing the ethnic identity scale using Eriksonian and social identity perspectives. Identity: An International Journal of Theory and Research, 4, 9-38.

Usborne, E., \& Taylor, D. M. (2010). The role of cultural identity clarity for self-concept clarity, self-esteem, and subjective well-being. Personality and Social Psychology Bulletin, 36(7), 883-897. doi: 10.1177/0146167210372215

van der Zee, K., van Oudenhoven, J. P., \& de Grijs, E. (2004). Personality, threat, and cognitive and emotional reactions to stressful intercultural situations. Journal of Personality, 72, 1069-1096.

van Hoof, A. (1999). The identity status field re-reviewed: An update of unresolved and neglected issues with a view on some alternative approaches. Developmental Review, 19(4), 497-556. doi: 10.1006/drev.1999.0484

Vleioras, G., \& Bosma, H. A. (2005). Are identity styles important for psychological wellbeing? Journal of Adolescence, 28(3), 397-409. doi:

10.1016/j.adolescence.2004.09.001

Ward, C. (2006). Acculturation, identity and adaptation in dual heritage adolescents. International Journal of Intercultural Relations, 30(2), 243-259. doi: 10.1016/j.ijintrel.2005.09.001

Ward, C. (2008). Thinking outside the Berry boxes: New perspectives on identity, acculturation and intercultural relations. International Journal of Intercultural Relations, 32(2), 105-114. doi: 10.1016/j.ijintrel.2007.11.002

Ward, C., Bochner, S., \& Furnham, A. (2001). The psychology of culture shock (2nd ed.). London: Routledge.

Ward, C., \& Kennedy, A. (1994). Acculturation Strategies, Psychological Adjustment, and Sociocultural Competence during Cross-Cultural Transitions. International Journal of Intercultural Relations, 18(3), 329-343. doi: 10.1016/0147-1767(94)90036-1 
Ward, C., \& Kus, L. (2012). Back to and beyond Berry's basics: The conceptualization, operationalization and classification of acculturation. International Journal of Intercultural Relations, 36(4), 472-485. doi: 10.1016/j.ijintrel.2012.02.002

Ward, C., Leong, C. H., \& Low, M. L. (2004). Personality and sojourner adjustment: An exploration of the big five and the cultural fit proposition. Journal of Cross-Cultural Psychology, 35(2), 137-151. doi: 10.1177/0022022103260719

Ward, C., \& Rana-Deuba, A. (1999). Acculturation and adaptation revisited. Journal of Cross-Cultural Psychology, 30(4), 422-442. doi: 10.1177/0022022199030004003

Ward, C., \& Searle, W. (1991). The impact of value discrepancies and cultural identity on psychological and sociocultural adjustment of sojourners. International Journal of Intercultural Relations, 15(2), 209-225. doi: 10.1016/0147-1767(91)90030-K

Ward, C., Stuart, J., \& Kus, L. (2011). The construction and validation of a measure of ethnocultural identity conflict. Journal of Personality Assessment, 93(5), 462-473. doi: $10.1080 / 00223891.2011 .558872$

Watson, P. J., \& Morris, R. J. (2005). Spiritual experience and identity: Relationships with religious orientation, religious interest, and intolerance of ambiguity. Review of Religious Research, 46(4), 371-379. doi: 10.2307/3512167

Wheeler, H. A., Adams, G. R., \& Keating, L. (2001). Binge eating as a means for evading identity issues: The association between an avoidance identity style and bulimic behavior. Identity: An International Journal of Theory and Research, 1, 161-178. doi: 10.1207/S1532706XID0102_04

White, J. M., \& Jones, R. M. (1996). Identity styles of male inmates. Criminal Justice and Behavior, 23(3), 490-504. doi: 10.1177/0093854896023003005

Yakhnich, L. (2008). Immigration as a multiple-stressor situation: Stress and coping among immigrants from the former Soviet Union in Israel. International Journal of Stress Management, 15(3), 252-268. doi: 10.1037/A0013002

Yakhnich, L., \& Ben-Zur, H. (2008). Personal Resources, Appraisal, and Coping in the Adaptation Process of Immigrants From the Former Soviet Union. American Journal of Orthopsychiatry, 78(2), 152-162. doi: 10.1037/0002-9432.78.2.152

Yijala, A., Lonnqvist, J. E., Jasinskaja-Lahti, I., \& Verkasalo, M. (2012). Values as predictors of anticipated socio-cultural adaptation among potential migrants from Russia to Finland. Journal of Community \& Applied Social Psychology, 22(2), 95-110. doi: 10.1002/Casp.1104 
Ying, Y. W., Lee, P. A., \& Tsai, J. L. (2007). Attachment, sense of coherence, and mental health among Chinese American college students: Variation by migration status. International Journal of Intercultural Relations, 31(5), 531-544. doi: 10.1016/j.ijintrel.2007.01.001

Zamboanga, B. L., Schwartz, S. J., Jarvis, L. H., \& Van Tyne, K. (2009). Acculturation and substance use among Hispanic early adolescents: Investigating the mediating roles of acculturative stress and self-esteem. Journal of Primary Prevention, 30(3-4), 315-333. doi: 10.1007/s10935-009-0182-Z 